LEAD ARTICLE

Acta Cryst. (1995). B51, 115-148

\title{
Understanding and Control of Nucleation, Growth, Habit, Dissolution and Structure of Two- and Three-Dimensional Crystals Using 'Tailor-Made' Auxiliaries
}

\author{
By Isabelle Weissbuch, Ronit Popovitz-Biro, Meir Lahav and Leslie Leiserowttz \\ Department of Materials and Interfaces, The Weizmann Institute of Science, Rehovot 76100, Israel
}

(Received 24 March 1994, accepted 25 October 1994)

\begin{abstract}
Tailor-made auxiliaries for the control of nucleation and growth of molecular crystals may be classified into two broad categories: inhibitors and promoters. Tailor-made inhibitors of crystal growth can be used for a variety of purposes, which include morphological engineering and etching, reduction of crystal symmetry, assignment of absolute structure of chiral molecules and polar crystals, elucidation of the effect of solvent on crystal growth, and crystallization of a desired polymorph. As for crystal growth promoters, monolayers of amphiphilic molecules on water have been used to induce the growth of a variety of three-dimensional crystals at the monolayer-solution interface by means of structural match, molecular complementarity or electrostatic interaction. A particular focus is made on the induced nucleation of ice by mono-
\end{abstract}

Leslie Leiserowitz obtained his PhD in 1966, working on thermochromy in the group of the late Gerhard Schmidt at the Weizmann Institute of Science. He then moved to the Organic Chemistry Institute of the University of Heidelberg when he began a systematic investigation of the structural patterns and physical properites of organic crystals. Meir Lahav obtained his PhD in 1967 in solid-state chemistry, also in Gerhard Schmidt's research group. He did postdoctoral research with Paul Bartlett at Harvard. On his return to Israel, he embarked on a program for the design of systems for the generation and amplification of optical activity via crystallization. It was during this period that the Lahav-Leiserowitz collaboration started, with studies of photochemical reactions in solids. This team-work continued with a study of crystal nucleation, growth and dissolution in the presence of tailor-made auxiliaries. Ronit Popovitz-Biro received her PhD in solid-state chemistry in 1979, working in the research group of $M$. Lahav. After a posidoctoral period at the University of California she returned to the Weizmann Institute. She has since directed her efforts at the oriented growth of crystals via Langmuir monolayers and the design of multilayer films. Isabelle Weissbuch received her PhD in chemistry in 1985 at the Weizmann Institute of Science. She has continued to work at the Weizmann Institute where her main interest has been solid-state chemistry, with emphasis on the design and use of auxiliaries for the control of crystal nucleation, morphology and polymorphism, as well as structure determination of mono- and multilayer films.

(C) 1995 International Union of Crystallography Printed in Great Britain - all rights reserved layers of water-insoluble aliphatic alcohols. The twodimensional crystalline structures of such monolayers have been studied by grazing incidence $X$-ray diffraction. It has become possible to monitor, by this method, the growth, dissolution and structure of self-aggregated crystalline monolayers, and indeed multilayers, affected by the interaction of solvent molecules in the aqueous subphase with the amphiphilic headgroups, and by the use of tailor-made amphiphilic additives.

\section{Introduction}

Although crystals and crystallization as a process play an important role in pure and applied science, our ability to design crystals with desired properties, and our control over crystallization based on structural understanding are still limited. Indeed our ignorance of the overall process of crystal growth is brought to the fore by comparison with the controlled crystal growth provided in biomineralization. Organisms can mold crystals of specific morphologies, sizes and orientations in the form of composites of minerals and organic materials with characteristics vastly different from those of their inorganic counterparts (Löwenstam \& Weiner, 1989; Addadi \& Weiner, 1992). Nevertheless, the technological advances in controlling the microstructure of inorganic materials on the subnanometer scale have led to tremendous success in the preparation of ceramics, synthetic layered microstructures and semiconducting materials. Paradoxically, advances in the field of organic materials have not been as striking, although the techniques for the preparation of films such as Langmuir-Blodgett and self-assembled multilayers on solid support hold promise as pyroelectric, piezoelectric and frequency doubling devices etc. The thread that binds these diverse topics involves molecular interactions at interfaces.

The surfaces of a growing crystal can be thought of as composed of 'active sites' which interact stereospecifically with molecules in solution, in a manner similar to enzyme-substrate or antibody-antigen interactions. The repetitive arrangements at crystal surfaces, and the knowledge we have of their structures, offer simpler 
means to pinpoint molecular interactions. It is possible to use the concept of molecular recognition at interfaces to address open questions in the areas of crystal nucleation and growth, crystal polymorphism, and interactions with the growth environment.

Landmark papers for the prediction of crystal morphology were provided by Hartman \& Perdok $(1955 a, b)$, Hartman (1973) and Hartman \& Bennema (1980), who laid the ground rules for a quantitative determination of crystal morphology, based on the internal crystal structure. Regarding the effect of additives and solvent on crystal growth and morphology, a variety of important experimental studies have been reported in the latter half of this century. For example, Wells (1946) performed elegant experiments particularly on polar crystals to demonstrate the effect of solvent on crystal growth and shape. Changes in habit induced in $\mathrm{NaCl}$ crystals by the presence of $\alpha$-amino acids were reported by Fenimore \& Thraikill (1949). Buckley (1951) described a variety of crystal habit modifications, in particular the effect of dyes which led to changes in morphology and to colored inclusions known as hourglass or Maltese cross shapes. Whetstone (1954) explained the nature of such dye adsorption phenomena in inorganic crystals. He invoked a structural match involving Coulomb interactions between the additive and the structure of the crystal surface onto which adsorption occurred. In the case of molecular crystals, the sugar technologists (Smythe, 1967, 1971; van Hook, 1968, 1981; Mantovani, Gilli \& Faglioli, 1967) studied the effect of raffinose on the growth of sucrose during extraction of the latter from molasses. By carrying out kinetic studies, they established that raffinose hinders growth along specific directions of sucrose crystals and proved the presence of its selective occlusion. The control, however, on nucleation, growth, dissolution and morphology of molecular crystals remained primarily a matter of ' $\mathrm{mix}$ and try'. There was no general approach for the design of additives targeted for binding to particular crystalline phases or preselected faces of any crystal. It was not, and still is not, possible to wield the exquisite control mother nature exerts in the field of biological mineralization.

In recent years a stereochemical approach has been adopted involving the growth and dissolution of molecular crystals in the presence of 'tailor-made' auxiliaries, which interact with specific crystal faces and may be crystal growth inhibitors or nucleation promoters (Addadi et al., 1985; Weissbuch, Addadi, Lahav \& Leiserowitz, 1991). With the assistance of such molecular auxiliaries it is possible to examine a variety of processes. We list first those which have involved crystal growth inhibitors. Crystals may be engineered with desired morphologies (Berkovitch-Yellin, Addadi, Idelson, Lahav \& Leiserowitz, 1982). A correlation may be made between the chirality of tailor-made additives and the macroscopic properties of affected host crystals. By such means, the absolute configuration of chiral molecules or polar crystals may be assigned (Addadi, BerkovitchYellin, Weissbuch, Lahav \& Leiserowitz, 1986). With occlusion of the additive into the crystal, the symmetry of the crystal may be reduced, thus making a 'centrosymmetric' crystal emit a second harmonic signal on irradiation with a laser beam (Weissbuch, Lahav, Leiserowitz, Meredith \& Vanherzeele, 1989). Molecular crystals can be etched at desired faces on dissolution (Shimon, Lahav \& Leiserowitz, 1985, 1986) and the effect of solvent on crystal growth may be elucidated (Wireko et al., 1987; Shimon, Vaida, Addadi, Lahav \& Leiserowitz, 1990, 1991). Crystallization of a desired phase may be induced for the resolution of chiral molecules (Addadi, van Mil \& Lahav, 1982; Addadi, Weinstein, Gati, Weissbuch \& Lahav, 1982), or for the precipitation of a polar crystal polymorph (Staab, Addadi, Leiserowitz \& Lahav, 1990).

With tailor-made auxiliaries in the form of crystal nucleation promoters it has proven possible to engineer two-dimensional crystalline monolayers composed of amphiphilic molecules on aqueous solutions to induce, by epitaxy, nucleation of crystals at the monolayersolution interface (Landau, Popovitz-Biro, Levanon, Leiserowitz \& Lahav, 1986; Landau et al., 1989) and, as a corollary, to acquire information relevant to the mechanism of nucleation of crystals in biological environments (Mann, 1988; Mann, Heywood, Rajam \& Birchall, 1988). The crystallinity of such Langmuir monolayers on the water surface has been demonstrated by grazing incidence $\mathrm{X}$-ray diffraction measurements, also leading to structure determination almost at the atomic level (Jacquemain et al., 1990). The knowledge acquired from such induced crystallization and diffraction experiments led to the use of alcohol monolayers for induced nucleation of ice (Gavish, Popovitz-Biro, Lahav \& Leiserowitz, 1990; Popovitz-Biro, Wang, Majewski, Leiserowitz \& Lahav, 1994; Wang et al., 1994). The monolayers on the liquid surface can also be used for studying the effect of solvent on the formation, growth and structure of polymorphic arrangements of twodimensional crystallites, information not easily deduced from three-dimensional crystals.

Here we present a birds-eye view of all these different, yet connected, topics.

\section{Morphological crystal engineering}

The habit of a crystal is defined by the relative rates of its growth in different directions; the faster the growth in a given direction, the smaller the face developed perpendicular thereto. Consequently, when growth is inhibited in a direction perpendicular to a given face, the area of this face is expected to increase relative to the areas of other faces of the same crystal. Differences in the relative surface areas of the various faces carı therefore 
be directly correlated to the inhibition in different growth directions. Dramatic morphological changes observed during the growth of organic crystals in the presence of additives revealed a high degree of specificity in the interaction of the foreign material with the different structures of surfaces of the crystalline matrix. Such a change in crystal morphology is depicted in Scheme 1.

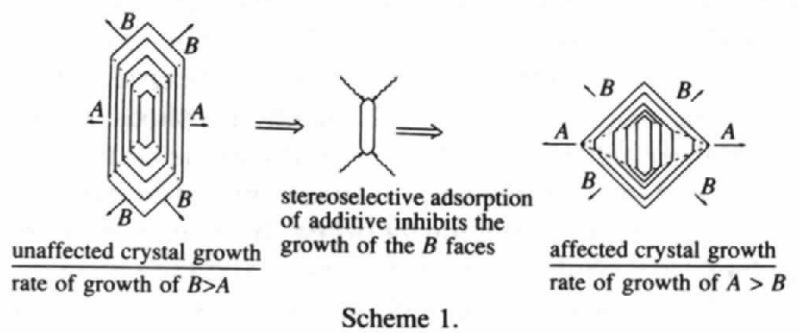

As mentioned previously, the role played by impurities on the habit modification of crystals (Kern, 1953) has long attracted the attention of crystal growers. However, such studies did not, in the main, involve morphological change by design. This became possible with the use of 'tailor-made' additives. From a systematic study of a variety of organic compounds crystallized in the presence of additives of molecular structure similar to the structures of the corresponding substrate molecules (Addadi et al., 1985), and so labeled 'tailor-made' additives (or auxiliaries), it was possible to deduce a stereochemical correlation between the structures of the affected crystal surfaces and the molecular structure of the additive. We could infer that the 'tailor-made' additive is adsorbed on the growing crystals, but only at certain surfaces and then with the part of the adsorbate that differs from that of the substrate emerging from the crystal. This selective adsorption of the tailor-made additive at crystal faces and concomitant inhibition of their growth is depicted in Scheme 2. Once this mechanism was established, it became possible to exploit it to systematically modify the morphology of

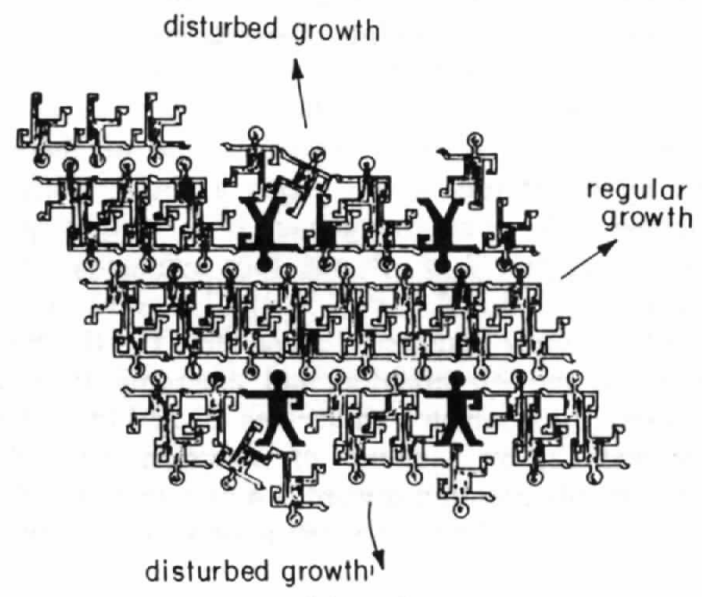

Scheme 2. crystals by tailoring additives that bind at preselected faces and thus inhibit growth in a predictable manner.

Benzamide, $\mathrm{C}_{6} \mathrm{H}_{5} \mathrm{CONH}_{2}$, provides an example where the host-additive interactions at the growing solid-liquid interface can be pinpointed and correlated with the change in crystal morphology (Berkovitch-Yellin, Addadi, Idelson, Lahav \& Leiserowitz, 1982). This compound crystallizes from ethanol as plates [Fig. 1(a)], in the monoclinic space group $P 2_{1} / c$. The molecules in the crystal form hydrogen-bonded cyclic dimers, further interlinked by hydrogen bonds to yield ribbons parallel to the $b$-axis [Fig. 2(a)]. The ribbons are stacked along the $5.6 \AA a$-axis to form stable (001) layers. These tightly packed layers juxtapose along the $c$-direction, interacting via weak van der Waals interactions between phenyl groups, thus accounting for the $\{001\}$ plate-like shape of the crystals. Benzoic acid additive, $\mathrm{C}_{6} \mathrm{H}_{5} \mathrm{COOH}$, adopting the stable $\mathrm{O}=\mathrm{C}-\mathrm{OH}$ synplanar conformation, can replace a molecule of benzamide at the end of a ribbon [Fig. 2(a)], however, at the site of the additive, an attractive $\mathrm{N}-\mathrm{H} \cdots \mathrm{O}$ bond $\left(-25 \mathrm{kj} \mathrm{mol}^{-1}\right)$ is replaced by repulsion $\left(4-8 \mathrm{kj} \mathrm{mol}^{-1}\right)$ between adjacent oxygen lone-pair electrons of the bound additive molecule and of the oncoming benzamide molecule, leading to an overall loss in energy of $29-33 \mathrm{kj} \mathrm{mol}^{-1}$ (BerkovitchYellin et al., 1985). As predicted, the presence of benzoic acid in solution inhibits the growth of the benzamide crystals along b, transforming the pure plate-like crystals into needles elongated along the $a$-axis [Fig. 1(b)]. Inhibition of growth along the $a$ direction was accomplished by adding $o$-toluamide, $o$ $\mathrm{H}_{3} \mathrm{CC}_{6} \mathrm{H}_{4} \mathrm{CONH}_{2}$, to the crystallization solution. The crystals consistently grew as bars elongated in $b$ [Fig. $1(c)$ ]. The additive $o$-toluamide can easily be adsorbed in the hydrogen-bonding chain without disturbing growth in the $b$-direction. The $o$-methyl group emerges, from the $(10 \overline{4})$ side face [Fig. $2(b)$ ], and thus interferes with

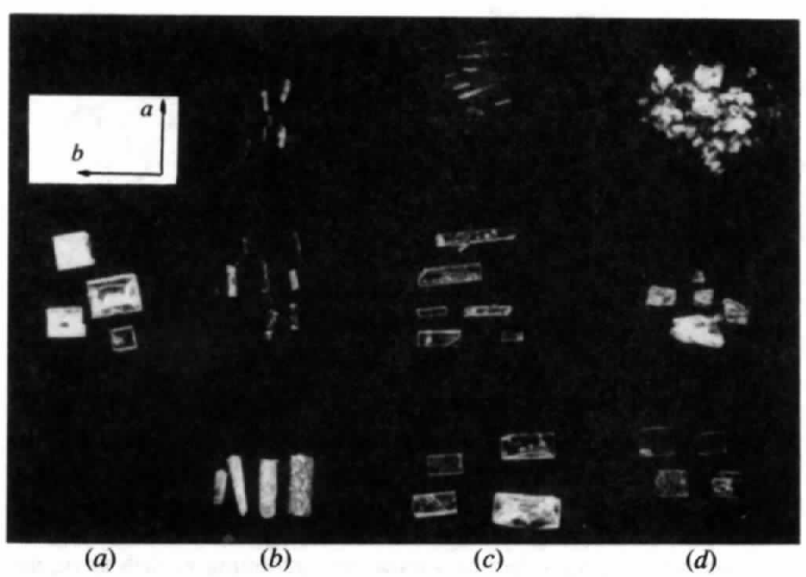

Fig. 1. Crystals of benzamide: $(a)$ pure, $(b)-(d)$ grown in the presence of additives: $(b)$ benzoic acid, $(c) o$-toluamide and $(d) p$-toluamide. 
growth in the $a$-direction, along which the dimers are stacked. The relative orientation of benzamide at the $\{001\}$ faces is shown in Fig. 2(c). Thinner and thinner plates [Fig. $1(d)$ ] are obtained by adding increasing amounts of $p$-toluamide, $p-\mathrm{H}_{3} \mathrm{CC}_{6} \mathrm{H}_{4} \mathrm{CONH}_{2}$, whose $p$ methyl substituent perturbs the already weak van der Waals interactions between the phenyl layers in the $c$ direction [Fig. 2(c)].

The present approach which is based upon specific molecular interactions at the solid-liquid interface was applied to a variety of molecular crystals, including $\alpha$ amino acids, dipeptides, primary and secondary amides, carboxylic acids, sugars, steroids etc. It therefore appears to be general and has since been successfully applied to a large class of materials, be they organic (Lewtas, Tack, Beiny \& Mullin, 1991; Skoulika, Michaelidis \& Aubry, 1991; Antinozzi, Brown \& Purich, 1992), inorganic (Black et al., 1991; Cody \& Cody, 1991; Ristic, Sherwood \& Wojeshowski, 1994) or biological (Markman, Elias, Addadi, Cohen, \& Berkovitch-Yellin, 1992).

\section{3. 'Tailor-made' etchants for organic crystals}

Growth and dissolution are not reciprocal processes. Growth forms are bound by the slowest growing faces,

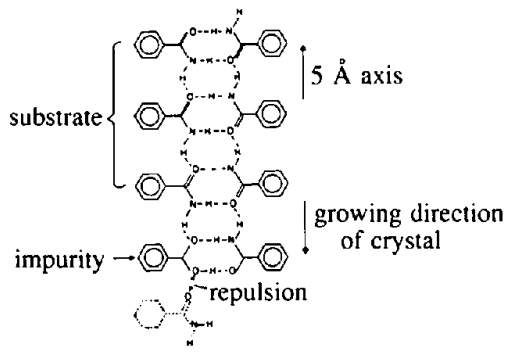

(a)

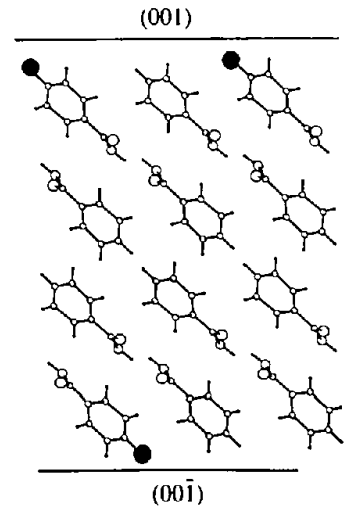

(c)

Fig. 2. (a) Schematic representation of the ribbon motif of hydrogenbonded dimers of benzamide molecules interlinked along the $5 b$-axis, showing the effect of benzoic acid inhibiting growth along the $b$ direction. (b) Packing arrangement of benzamide, viewed along the $b$-axis, showing the effect of $a$-toluamide inhibiting growth along the $a$-direction. (c) Packing arrangement of benzamide, viewed along the $b$-axis, showing the effect of $p$-toluamide inhibiting growth along the $c$-direction dissolution forms by the fastest dissolving directions (de Boer, 1993). Nevertheless, we found that a 'tailor-made' inhibitor of growth of a given crystal face affects the rate of dissolution of that same crystal face (Shimon, Lahav \& Leiserowitz, 1985, 1986).

It has long been recognized that partial dissolution of crystals in the presence of additives may induce the formation of well shaped etch-pits on particular faces of the crystal. For example, when crystals of calcite were partially dissolved in the presence of D- or L-tartaric, -malic or -lactic acids, etch-pits with enantiomorphous shapes were observed (Honess, 1927). Owing to the complexity of the etching mechanism, no simple model involving a stereochemical correlation between the structure of the etchant, that of the to-be-etched face, and the symmetry and the geometry of the etch-pit, had been evolved, so that selection of etchants was done generally by trial and error.

Crystal dissolution invariably begins at sites of emerging dislocations (Horn, 1952; Gevers, Amelinckx \& Dekeyser, 1952). At such sites more than one type of facial surface is exposed to the solvent. When an additive that binds selectively to a given face of the crystal is present in the solution, the crystal dissolves in different directions at rates that differ from those in the pure solution. Subsequently, etch-pits are formed at the dislocation centers on those faces at which the additives are bound. It is also correct to say that there is a difference between etching and dissolution. By inhibiting the latter, the former is made more prominent. The possibility of designing molecules which can interact stereospecifically with a given face of a crystal should, in principle, make those molecules efficient etchants of the face. Therefore, it was anticipated that the same molecules, which act as 'tailor-made' growth inhibitors of a given face, operate as 'tailor-made' etchants of the same face when the crystal is partially dissolved. The generality of this approach was tested on several molecular crystals (Shimon, Lahav \& Leiserowitz, 1985, 1986), which we illustrate with one example.

We consider the packing characteristics of the $\alpha$ form of glycine, ${ }^{+} \mathrm{H}_{3} \mathrm{NCH}_{2} \mathrm{CO}_{2}^{-}$, a prochiral molecule containing two $\mathrm{H}$ atoms at the central $\mathrm{C}$ atom that are enantiotopic, namely, replacing one of these $\mathrm{H}$ atoms by a different group yields a chiral molecule. $\alpha$-Glycine packs in an arrangement [Fig. 3(a)] of spacegroup symmetry $P 2_{1} / n$. Of the four symmetry-related molecules [1, 2, 3 and 4, Fig. 3(a)], (1) and (2), related by twofold screw symmetry, have their $\mathrm{C}-\mathrm{H}_{\mathrm{re}}$ bonds pointing in the $+b$-direction and emerging from the $(010)$ face. By symmetry, molecules (3) and (4), related to (1) and (2) by a center of inversion, have their $\mathrm{C}-\mathrm{H}_{\mathrm{si}} *$ bonds pointing toward $-b$ and emerging from the $(0 \overline{1} 0)$ face. Only the $(R)$-amino acid additives

\footnotetext{
* Note that we have used the Prelog \& Helmchen (1982) nomenclature to specify the diastereotopic $\mathrm{C}-\mathrm{H}$ ligands.
} 
can substitute a glycine molecule at the (1) and (2) sites, and then only on the $(010)$ face, while only $(S)$ amino acids can be adsorbed at sites (3) and (4) on the $(0 \overline{1} 0)$ face. This constraint arises from the steric requirement that the additive molecule be recognized on the $\{010\}$ surfaces as a 'host' molecule so that the $\alpha$-amino acid side chain can replace the $\mathrm{H}$ atom of glycine only at the $\mathrm{C}-\mathrm{H}$ bond emerging from the crystal surface. Otherwise, repulsion would occur between the additive and the surrounding molecules on the crystal surface. $\alpha$-Glycine crystallizes from water as bipyramids [Fig. 3(b)]. Crystallization experiments in the presence of $(R)$ - $\alpha$-amino acid additives led to the formation of pyramids with a (010) basal plane, because growth in the $+b$ direction is inhibited [Fig. 3(c)]. (S)- $\alpha$-Amino acid additives induce the enantiomorphous morphology [Fig. 3(d)]. Racemic $\alpha$-amino acid additives cause the formation of $\{010\}^{*}$ plates [Fig. 3(e)], because growth

* Note that the symbol $\{h k l\}$ refers to all the symmetry-related faces, whereas $(h k l)$ refers to only the face specified by $h, k, l$.

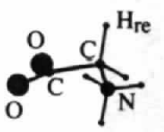

(010)

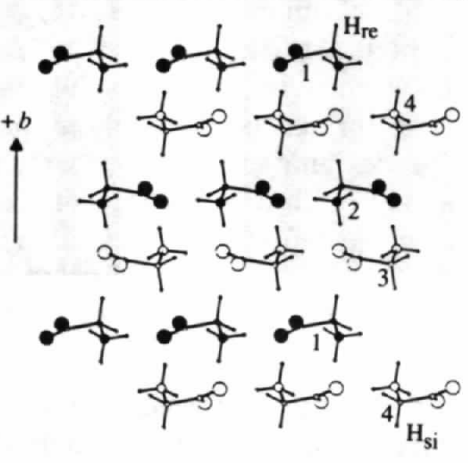

(010)

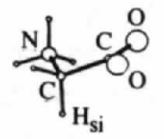

(a)

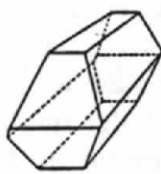

(b)

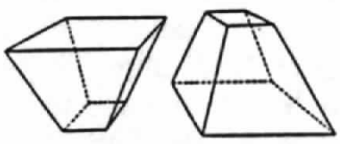

(c)

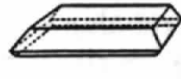

(e)
Fig. 3. (a) Packing arrangement of $\alpha$-glycine viewed along the $a$-axis showing the four symmetry-related molecules; the $\mathrm{C}-\mathrm{H}_{\mathrm{re}}$ bonds, almost parallel to $b$, of the molecules point towards the $(010)$ face and the corresponding $\mathrm{C}-\mathrm{H}_{\mathrm{si}}$ bonds towards the $(0 \overline{1} 0)$ face. $(b)-(e)$ Crystal morphologies of $\alpha$-glycine when grown in the presence of: (b) no additive, (c) $R$ - $\alpha$-amino acids, (d) $S$ - $\alpha$-amino acids and (e) racemic $\alpha$-amino acid additives. at both the $+b$ and $-b$ sides of the crystal is inhibited (Weissbuch et al., 1983).

The faces relevant to the etching process are of the type $\{010\}$, being those inhibited on growth. Platelike crystals of glycine with such well developed faces were submitted to partial dissolution in an undersaturated solution of glycine containing variable amounts of other $\alpha$-amino acids. In the presence of racemic additive, the rate of dissolution was much slower than in their absence. Moreover, the inhibition was more pronounced, the bulkier the additive side chain (Shimon, Lahav \& Leiserowitz, 1986; Shimon, Zbaida, Addadi, Leiserowitz \& Lahav, 1988). When resolved $R$-alanine, ${ }^{+} \mathrm{H}_{3} \mathrm{NCH}\left(\mathrm{CH}_{3}\right) \mathrm{CO}_{2}^{-}$, was present in the solution, well developed etch-pits were formed only on the $(010)$ face [Fig. 4(a)]. These pits exhibit twofold morphological symmetry with surface edges parallel to the $a$ - and $c$ axes of the crystal. The enantiotopic ( $0 \overline{1} 0)$ face dissolved smoothly [Fig. 4(b)], exactly as it does when the crystal is dissolved in an undersaturated solution of pure glycine. As expected, $S$-alanine induced etch-pits on the $(0 \overline{1} 0)$ face. Racemic alanine etched both opposite $\{010\}$ faces. The well established role played by dislocations for inducing etch-pit formation was also demonstrated. A pure crystal of glycine was cleaved through its basal plane to expose the $(010)$ and $(0 \overline{1} 0)$ faces. These two

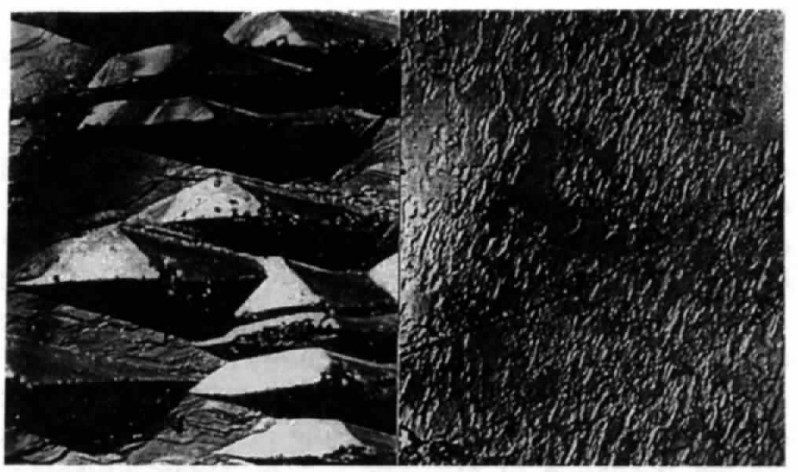

(a)

(b)

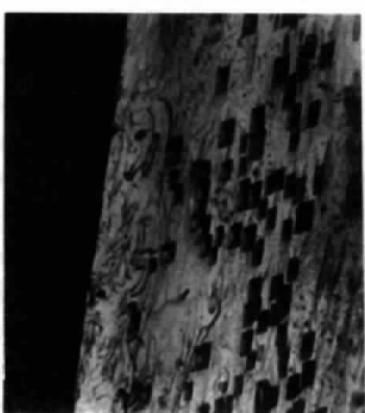

(c)

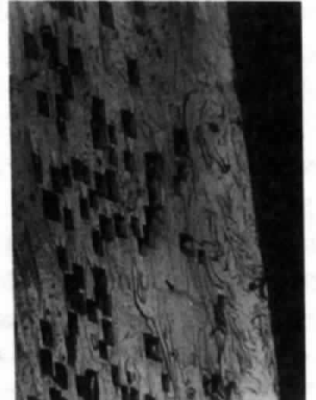

(d)
Fig. 4. Optical microscope photographs of the $\{010\}$ faces of an $\alpha$ glycine crystal after etching: $(a)$ and $(b)$ the $(010)$ and $(0 \overline{1} 0)$ faces as etched by $R$-alanine; $(c)$ and $(d)$ the $(010)$ and $(0 \overline{1} 0)$ faces of a cleaved crystal after etching with racemic alanine. 
parts of the crystal, on being etched with racemic alanine additive, displayed etch-pits in equivalent regions of the two cleaved faces [Figs. $4(c)$ and $(d)$ ].

The same type of chiral selectivity holds for an enantiomorphous pair of crystals dissolved in the presence of a chiral-resolved molecule, when only one of the enantiomers is etched. Such enantioselective etching experiments provide a simple method of differentiating between enantiomorphous single crystals which do not express hemihedral faces. The method has been demonstrated for a class of racemic $\alpha$-amino acids that crystallize as conglomerate mixtures (i.e. each enantiomer crystallizes in its own enantiomorphous crystal), such as chiral-resolved glutamic acid. $\mathrm{HCl}$ and asparagine. $\mathrm{H}_{2} \mathrm{O}$ (Shimon, Lahav \& Leiserowitz, 1986). Moreover, it also proved possible to bring about a pronounced separation of enantiomorphs by the enantioselective dissolution of a conglomerate mixture in the presence of 'tailor-made' optically pure polymeric additives (Shimon, Zbaida, Addadi, Leiserowitz \& Lahav, 1988).

\section{Assignment of absolute configuration of crystals and molecules}

\subsection{Polar crystals}

The standard method for the direct assignment of the absolute configuration of polar crystals, composed of chiral, racemic or nonchiral molecules, was introduced by Bijvoet in 1948 with the use of anomalous X-ray scattering. Over the past decade, several independent methods for the assignment of the absolute configuration of polar crystals have been applied, including the effect of tailor-made molecular additives on crystal morphology and structure, as well as gas-solid reactions (Paul \& Curtin, 1987) and ferroelectric measurements in liquid crystals (Walba, Razuvi, Clark \& Parmer, 1988). The induced change in crystal morphology with tailor-made additives, the anisotropic distribution of the additive inside the host crystal, and the role played by additives for the formation of etch-pits can be taken advantage of for the assignment of the absolute configuration of chiral molecules and polar crystals. In this way it has been possible to assign the absolute molecular structure of a variety of molecular systems, such as $\alpha$-amino acids, sugars and steroids (Addadi, Berkovitch-Yellin, Weissbuch, Lahav \& Leiserowitz, 1986). Although one might argue that these studies have only confirmed what was already known, they demonstrate the interplay between molecular chirality and various macroscopic phenomena, and also satisfy the curiosity of students of stereochemistry with an inadequate knowledge of Xray scattering. Furthermore, the method has been applied to assign the absolute structures of polar crystals whose constituent molecules are either a racemic mixture or nonchiral, and may be the only viable method if the constituent molecules are hydrocarbons.
To demonstrate the method we first focus on polar crystals composed of chiral-resolved molecules. The method involves the principle of fixing the orientation of the constituent molecules vis-à-vis the polar axis, or axes, of the crystal and subsequently determining the absolute configuration of the chiral molecules. This principle is depicted in Scheme 3, which shows two enantiomorphous sets of hands arranged in a lattice, right hands forming an $(R)^{*}$ crystal and left hands forming an $(S)^{*}$ crystal. The fingers of the hands are exposed at the $(010)$ face of the right-handed 'molecules' and, by symmetry, at the $(0 \overline{1} 0)$ face for the left-handed 'molecules'. Thus, by determining at which face of the crystal specimen the fingers or wrists are exposed, the handedness of the constituent molecules may be assigned. This may be performed by applying the twostep adsorption-inhibition mechanism described above with appropriate tailor-made additives.

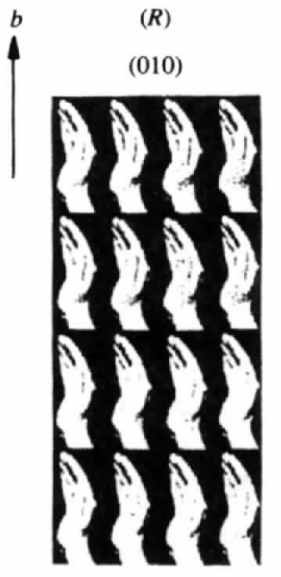

(010)

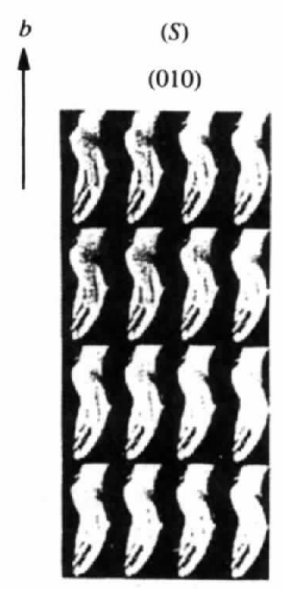

(010)
Scheme 3 .

We illustrate this approach with the example of $N$ - $(E$-cinnamoyl)alanine (Berkovitch-Yellin, Addadi, Idelson, Leiserowitz \& Lahav, 1982). Molecules of $N$ ( $E$-cinnamoyl)- $S$-alanine $(1 a)$ crystallize in space group $P 2_{1}$. The packing arrangement delineated by the crystal faces as grown from methanol solution is shown in Fig. 5. The molecules are arranged such that the carboxyl groups emerge at the two $\{1 \overline{1} 1\}$ faces and the $\mathrm{C}$ (chiral) $-\mathrm{H}$ bonds are directed along the $+b$ axis. In a study on the effect of tailor-made additives on the morphology of pure $N$-(E-cinnamoyl)-(S)-alanine [1a, Fig. $6(a)]$, it was found, as expected, that the methyl ester $(1 b)$, of the same absolute configuration as the host molecule $(1 a)$, induced large $\{1 \overline{1} 1\}$ faces [Fig. $6(b)$ ], since the $\mathrm{O}-\mathrm{CH}_{3}$ group replaces a $\mathrm{O}-\mathrm{H}$ group emerging from such faces. Tailor-made additive $N$ - $(E$ cinnamoyl)-( $R$-alanine $(1 c)$ of configuration opposite

\footnotetext{
*Note that for convenience the parentheses enclosing the chirality of the molecule specifies a crystal; absence thereof, such as $R, S$ or $R, S$, specifies the molecule only.
} 
to that of the host induced, as expected, a (010) face [Fig. 6(c)], because of an interchange of $\mathrm{CH}_{3}$ and $\mathrm{H}$ at the guest asymmetric carbon so that the $\mathrm{C}-\mathrm{CH}_{3}$ guest moiety replaces the emerging host $\mathrm{C}-\mathrm{H}$ bond.

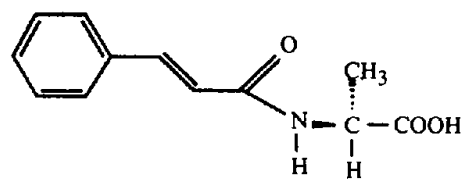

(1a)

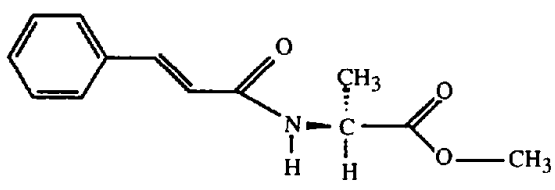

(1b)

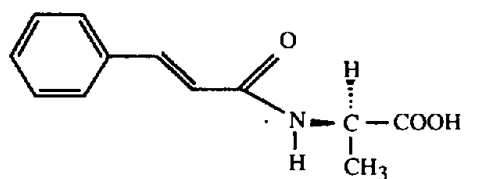

(1c)

It was found that solvent may act in a manner similar to the tailor-made additives, for example, by growing (1a) from acetic acid (Shimon, Vaida, Addadi, Lahav \& Leiserowitz, 1991) instead of methanol. Acetic acid is a solvent which can selectively bind at the exposed carboxylic acid groups of the two $\{1 \overline{1} 1\}$ faces, forming a hydrogen-bonded dimer (2a). Acetic acid can also bind to the $\mathrm{CHCO}_{2} \mathrm{H}$ moiety of cinnamoylalanine via a cyclic dimer $(2 c)$ on the $(010)$ face. The dimer $(2 b)$ is the motif adopted by acetic acid in its own crystal structure (Jonsson, 1971), forming a C-H...O(carbonyl) interaction (Taylor \& Kennard, 1982; Berkovitch-Yellin \& Leiserowitz, 1984). Crystallization of $N$-(E-cinnamoyl)alanine (1a) from glacial acetic acid yields crystals with the morphology shown in Fig. $6(d)$, in keeping with expectation.

A nice example of how anomers, interconvertible in solution, may be used for the assignment of absolute<smiles></smiles>

$(2 a)$

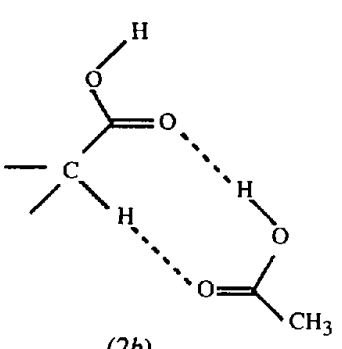

(2b)

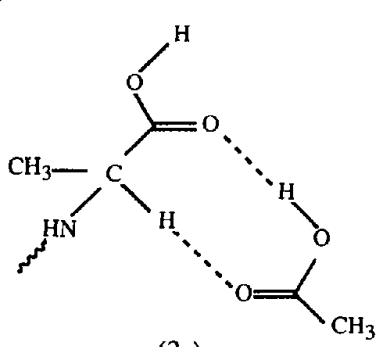

$(2 c)$ configuration is provided by the influence on the morphology of $\alpha$-lactose hydrate crystals by the anomeric $\beta$-lactose molecules present in solution (Michaelis \& van Kreveld, 1966; Visser \& Bennema, 1983).

Direct assignment of the absolute configuration of chiral-resolved molecules by the difference in wetting properties of hydrophobic and hydrophilic crystal faces was made possible (Wang, Lahav \& Leiserowitz, 1991) using crystals of the class belonging to chiral-resolved
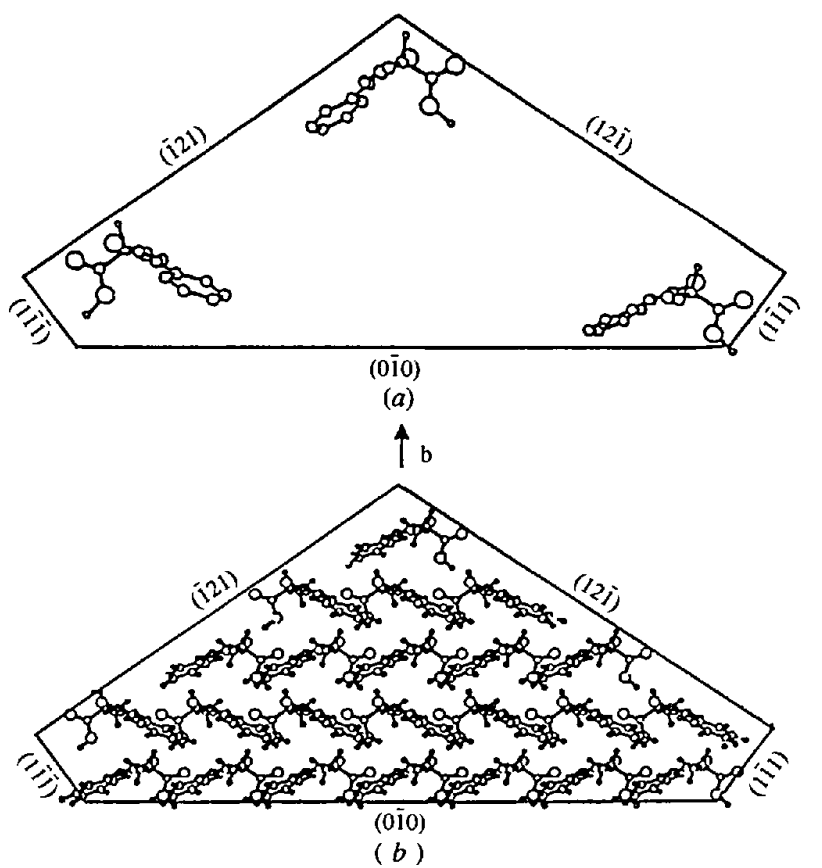

Fig. 5. Packing arrangement of $N$-(E-cinnamoyl)-(S)-alanine delineated by the faces observed in the pure crystal. To demonstrate the effect of the tailor-made additives, $(a)$ shows the orientation of only three host molecules vis-à-vis the crystal faces.

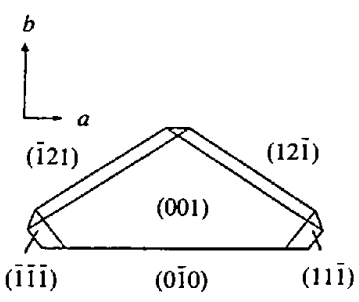

(a)

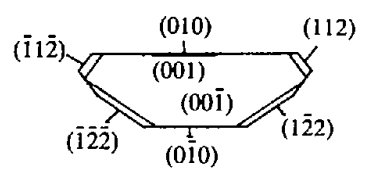

(c)

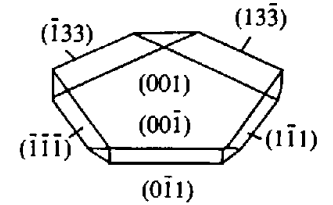

(b)

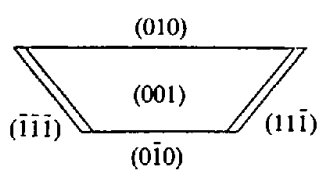

(d)
Fig. 6. Morphologies of measured crystals of $N$-(E-cinnamoyl)-(S)alanine. (a)-(c) refer to crystals grown from methanol: (a) pure crystal, (b) grown in the presence of the methyl ester $(b),(c)$ grown in the presence of $E$-cinnamoyl-(R)-alanine $(l c)$ and $(d)$ pure crystal grown from acetic acid. 
alkyl gluconamides, $\mathrm{C}_{n} \mathrm{H}_{2 n+1} \mathrm{NHCO}(\mathrm{CHOH})_{4} \mathrm{CHOH}$, $n=7-10$. The molecules crystallize as plates in an arrangement akin to that depicted in Scheme 3, namely head-to-tail, as shown in Fig. 7 (Zabel et al., 1986). Thus, one face of the plate crystal is hydrophobic and the opposite hemihedral face is hydrophilic. Contact angle measurements with a variety of polar solvents deposited on the opposite plate-like faces of specimen crystals could establish which face is hydrophobic and which is hydrophilic. This information fixes the orientation of the constituent molecules along the polar $b$-axis of the crystal specimen and consequently their chirality. These wettability experiments also led to crystal growth experiments on these systems to help determine the role of solvent on crystal growth, as discussed in a later section.

The last example involves etching experiments, which have the advantage that they may be performed on pure specimen crystals which had not been grown in the presence of a tailor-made additive. Here we describe the assignment of the absolute structure of racemic $(R, S)$-alanine by enantioselective etching (Shimon et al., 1986). This compound crystallizes in the polar space group $P n a 2_{1}$. In the crystal, which is needle-like along the $c$-axis, the $R$ - and $S$-molecules are oriented with respect to the polar needle $c$-axis so that the carboxylate $\mathrm{CO}_{2}^{-}$groups are exposed at one end of the polar axis and the amino $\mathrm{NH}_{3}^{+}$groups at the opposite end. The crystals exhibit different (hemihedral) faces at opposite ends of the $c$-axis in the sense that one end face is flat and the opposite capped. Conventional X-ray crystallography does not allow one to assign the absolute molecular orientation with respect to the polar axis, and so at which ends are the $\mathrm{CO}_{2}^{-}$and the $\mathrm{NH}_{3}^{+}$groups exposed. Etching

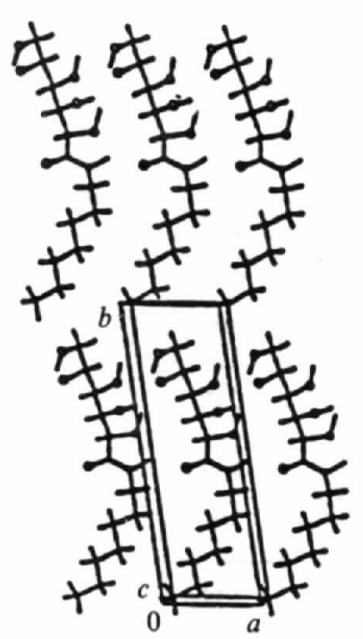

(a)

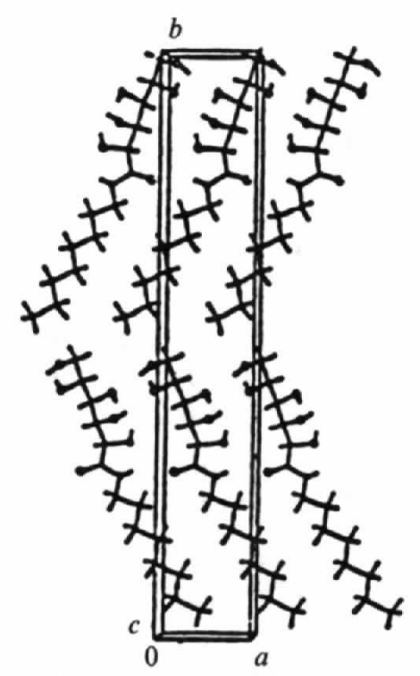

(b)
Fig. 7. Head-to-tail packing arrangement of (a) $N$-( $n$-heptyl)-Dgluconamide in space group $P 1$ and $(b) N$-(n-octyl)-D-gluconamide in space group $P 2_{1}$. has been successfully applied for such an assignment by making use of the well developed $\{210\}$ side faces of the needle. The orientations of the $(R)$ and $(S)$-molecules $v i s-d-v i s$ the four symmetry-related $\{210\}$ side faces for the two possible orientations of the crystal structure with respect to the polar axis are shown in Figs. 8(a) and $(b)$. Naturally, all four symmetry-related molecules are exposed at each of the four symmetry-related $\{210\}$ faces, but only one of the four molecules is oriented such that its $\mathrm{C}-\mathrm{CH}_{3}$ group emerges from a particular $\{210\}$ crystal face, as shown in Fig. 8. Once these orientations in a specimen crystal are determined, the absolute molecular arrangement vis- $\boldsymbol{a}$-vis the polar axis is assigned. Partial dissolution in the presence of $S$ - $\alpha$-amino acids such as serine, ${ }^{+} \mathrm{H}_{3} \mathrm{NCH}\left(\mathrm{CH}_{2} \mathrm{OH}\right) \mathrm{CO}_{2}^{-}$, or phenylalanine, ${ }^{+} \mathrm{H}_{3} \mathrm{NCH}\left(\mathrm{CH}_{2} \mathrm{C}_{6} \mathrm{H}_{5}\right) \mathrm{CO}_{2}{ }^{-}$, should replace an $(S)$-alanine substrate molecule at only the $(\overline{2} 10)$ and $(2 \overline{1} 0)$ faces for the orientation shown in Fig. 8(a), but would affect the

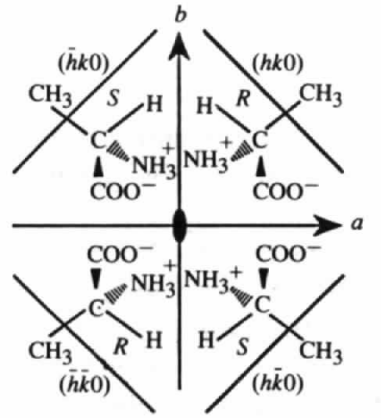

(A)

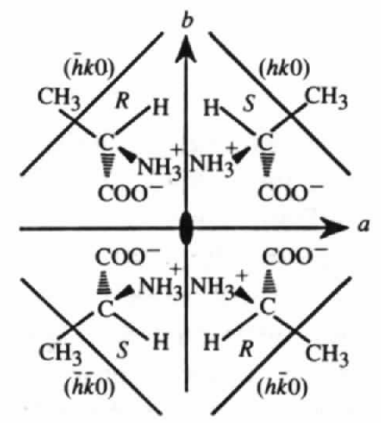

(B)
Fig. 8. Schematic ( $m m 2$ point group) representation of the orientation of the four molecules of $(R, S)$-alanine vis-d-vis the symmetryrelated $\{210\}$ faces, when viewed along the polar $c$-axis. These four molecules are oriented such that only one has its $\mathrm{C}-\mathrm{CH}_{3}$ group emerging from a given $\{210\}$ face. $(A)$ and $(B)$ represent the two alternative orientations of the molecules for the corresponding orientations of the polar crystal structure for a given direction of the $c$-axis.

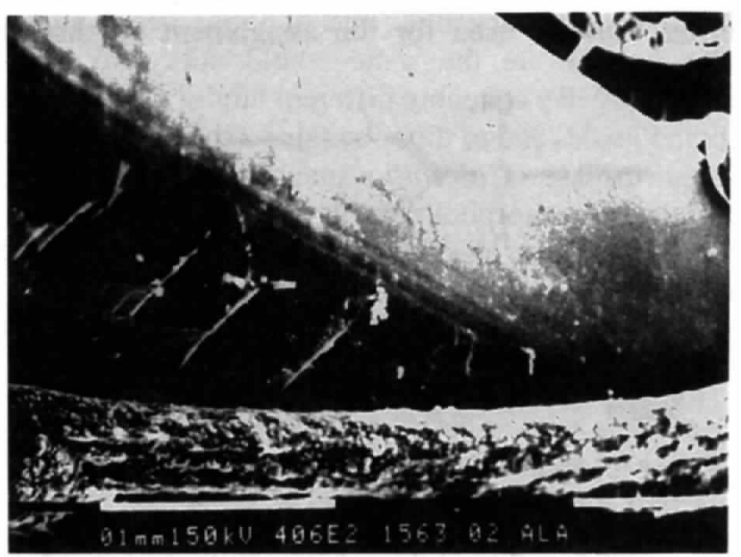

Fig. 9. Scanning electron micrograph of one of the $\{210\}$ side faces of $(R, S)$-alanine etched by $(S)$-threonine; only two adjoining faces are shown, one etched and the other smooth. 
(210) and $(\overline{2} \overline{1} 0)$ pair for the opposite orientation of Fig. $8(b)$. This expectation was demonstrated experimentally, etch-pits being formed on only one pair of $\{210\}$ side faces (Fig. 9), so fixing the absolute structure of the specimen crystal, and thus of all crystals grown from aqueous solution because the crystals exhibit hemihedral end faces.

The absolute orientation of specimen polar crystals of $(R, S)$-alanine could also be assigned from growth experiments in the presence of additives designed to affect either of the two polar ends of the crystal. Crystallization experiments in the presence of alanine ethyl ester that would bind at the fast growing $\mathrm{CO}_{2}^{-}$end of the crystal yielded much shorter needles. $N$-Methylalanine, which was expected to affect the slow-growing capped end of the crystal, yielded needles as long as from the pure solution, thus fixing the orientation of the molecules in the crystal (Shimon et al., 1986).

Growth experiments in the presence of additives, complemented by surface energy calculations, have also been used for assignment of absolute structure. For example, the absolute orientation of specimen polar crystals of $m$-chloronitrobenzene ( $m$-CNB) was determined by crystal growth experiments in the presence of $p$-chloronitrobenzene ( $p$-CNB) and $p$-dinitrobenzene ( $p$-DNB) additives (Chen, Garside, Davey, Maginn \& Matsuoka, 1994). The pure $m$-CNB crystals grown from melt and from acetone solutions do not exhibit hemihedral faces, although the space group is $P n a 2_{1}$. Growth in the presence of $p$-CNB and $p$-DNB yielded asymmetrically shaped crystals affected along the polar $c$-axis. Qualitative calculations of the binding energy of the additives to the crystal were in agreement with the experiment, insofar as the additive could be bound at only one specific end of the polar axis. Docherty, Roberts, Saunders, Black \& Davey (1993) calculated a polar morphology of urea (space group $P \overline{4} 2_{1} m$ ) which was in good agreement with the crystal shape obtained from sublimation. A classical type of energy calculation in which one assumes that the charge distribution of the molecules in the bulk and of those being attached at the surface are the same would not yield a polar morphology. By attaching different atomic charges to the bulk molecules and to those being attached at the surface, through the use of $a b$ initio quantum chemical calculations, the polar morphology was computed. However, the assignment of the absolute crystal structure of urea has not yet been confirmed by experimental methods. The crystals of urea grown from benzene or water, discussed in $\$ 7.4$, do not exhibit polar morphologies.

\subsection{Use of centrosymmetric crystals for assignment of molecular chirality}

In contrast to the situation for chiral crystals, it had not been generally appreciated that in centrosymmetric crystals the orientations of the constituent molecules are unambiguously assigned, with respect to the crystal axes, from a conventional structure determination. Thus, the known orientation of the two enantiomeric molecules in a centrosymmetric crystal can be exploited for the direct assignment of absolute configuration of chiralresolved molecules, provided the structural information embedded in the racemic crystal is transferred to a chiral additive molecule. The direct assignment of the absolute configuration of such chiral-resolved additives may thus be determined through the morphological changes they induce selectively on one set of enantiotopic faces of centrosymmetric crystals with appropriate packing features.

A prerequisite for application of this method is that within the centrosymmetric racemic crystal a specific functional group $A$ attached to an $R$-molecule points towards the faces $f \overline{1}$ and $f \overline{2}$, but not towards the opposite faces $f 1$ and $f 2$ (Scheme 4). By symmetry, the same functional group attached to an $S$-molecule will emerge at the enantiotopic faces $f 1$ and $f 2$, but not $f \overline{1}$ and $f \overline{2}$. Crystallization of a centrosymmetric crystal in the presence of a chiral additive $R^{\prime}$, in which $A$ is replaced by $Y$, designed so that it will fit in the site of an $R$ molecule on the growing crystal faces $f \overline{1}$ or $\bar{f}$, but not on the enantiotopic faces $f 1$ or $f 2$, will hinder growth in the $-b$ direction, but not in the $+b$ direction (Scheme 4). By virtue of symmetry, the enantiomeric additive $S^{\prime}$ will inhibit growth perpendicular to faces $f 1$ and $f 2$, while racemic additive $R^{\prime}, S^{\prime}$ will inhibit growth in both directions $+b$ and $-b$. This approach is not limited to racemic crystals, but the constituent molecules should at least be prochiral for ease of interpretation. For example, the absolute configuration of all naturally occurring $\alpha$ amino acids, except proline, may be assigned by their effect on the morphology of the $\alpha$-form of glycine, a prochiral molecule (Weissbuch et al., 1983). We shall illustrate the above principle making use of centrosymmetric crystals of $(R, S)$-serine to assign the absolute configuration of threonine as an additive (Weissbuch, Shimon et al., 1985).

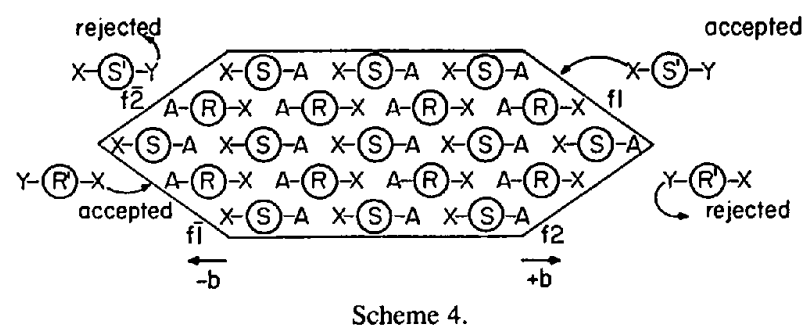

Racemic serine crystallizes in space group $P 21 / a$. Within the structure (Fig. 10), the $\mathrm{C}_{\beta} \rightarrow \mathrm{H}_{S \mathrm{i}}$ bond vector of the rigid methylene group of the $R$-serine molecule has a major component along $+b$ and, by symmetry, the $\mathrm{C}_{\beta} \rightarrow \mathrm{H}_{\mathrm{Re}}$ bond vector of the $S$-serine has a major component along $-b$. Thus, their replacement by methyl, 
as in threonine, will inhibit growth in the $b$-direction. That is, an $R$-threonine molecule with a side-chain $\beta$ carbon of chirality $S$ will inhibit growth along $+b$, while the $-\mathrm{CH}_{3}$ group of $S$-threonine will replace the $\mathrm{H}_{\mathrm{Re}}$ hydrogen of $S$-serine and hence inhibit growth along $-b$. $(R, S)$-serine forms tabular crystals with point symmetry 2/m [Fig. 11(a)]; the crystals affected by either $R$ - or $S$ threonine exhibit reduced morphological point symmetry 2 (the mirror plane is lost) and are enantiomorphous [Figs. 11(b) and $(c)$ ]. When $R, S$-threonine is used as the additive, the morphological point symmetry $2 / m$ is left

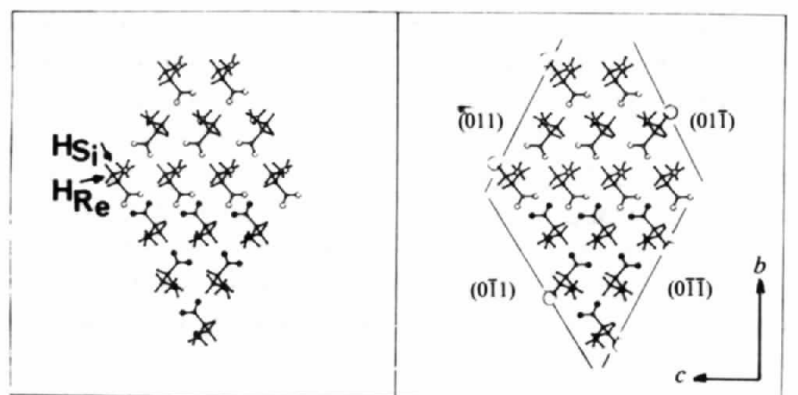

Fig. 10. Stereoscopic view of the packing of the $(R, S)$-serine crystal along the $a$-axis. Each $b c$-layer is composed of $R$ - or $S$-molecules only. For clarity, only half of each $R$ - (open circles) and $S$ - (filled circles) layer is shown. The positions of the four $\{011\}$ faces are shown with respect to the crystal structure. Threonine additive molecules have been stereospecifically inserted, with the $\beta$-methyl groups indicated by large circles.
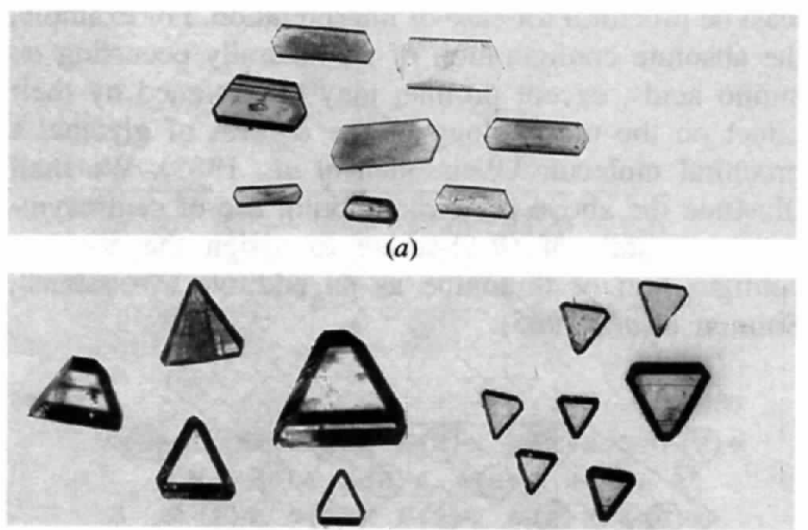

(b)

(c)

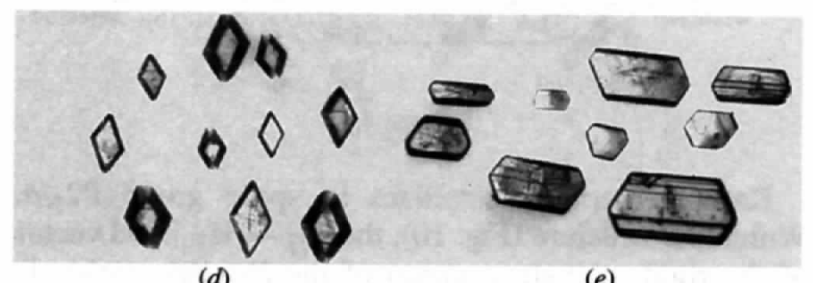

Fig. 11. Photographs of $(R, S)$-serine crystals which all have a common orientation of the lattice axes and a vertical $b$-axis: $(a)$ pure; $(b)-(e)$ grown in the presence of: (b) $R$-threonine, (c) $S$-threonine, (d) $R, S$ threonine and $(e) R, S$-allothreonine. unchanged because the effects induced by each additive separately combine. The crystals turn into rhombs, with a clear increase in the areas of the $\{011\}$ side faces relative to those of the pure form [Fig. 11(d)]. The changes in morphology seen with the additives in Figs. 11(b)-(d) with respect to that of the pure crystals [Fig. 11(a)] can be interpreted only in terms of selective adsorption of the $R$-enantiomer at faces (011) and (011), and of $S$ at $(0 \overline{1} 1)$ and $(0 \overline{1} \overline{1})$. According to calculated surface binding energies (Weissbuch, Shimon et al., 1985), $R$ threonine may easily be adsorbed only on faces (011) and $(01 \overline{1})$ and, by symmetry, $S$-threonine only on $(0 \overline{1} 1)$ and $(0 \overline{1} \overline{1})$. These results fix the absolute chirality of the chiral-resolved threonine additive.

\section{Symmetry lowering in crystals following selective occlusion of tailor-made auxiliaries}

Crystallization is a commonly used process for purification of inorganic and organic materials, and so the mode of occlusion of impurities or additives inside growing crystals is of paramount technological and theoretical importance. The results presented above, on the interaction of 'tailor-made additives' with the different faces of crystals, have bearing on the general question regarding the distribution and arrangement of occluded 'tailor-made' additives within crystals, indeed on the symmetry and structure of crystalline solid solutions.

A basic concept pertaining to this question is that, in general, the two-dimensional symmetry relating molecules at crystal surfaces is generally lower than the three-dimensional symmetry of the bulk crystal and that the surface structures of nonsymmetry-related faces have different arrangements. For simplicity we illustrate this aspect with a schematic arrangement of spacegroup symmetry $P 2_{1} / c$, shown in Fig. 12 . Note the corresponding symmetry operations of the $2 / \mathrm{m}$ point group given in the insert. The crystal is delineated by four symmetry-related diagonal faces, labeled $\{011\}$. At these four surfaces, the three symmetry relations belonging to space group $P 2_{1} / c$ (i.e. center of inversion, twofold screw and glide) are broken, thus their twodimensional symmetry is limited to translation, plane symmetry $p 1$. We now examine the effect of tailormade additive on these faces. One can easily see that an additive molecule, in black in Fig. 12, bearing an appropriately modified group, will be able to substitute for a substrate molecule at any diagonal face, say (011), but at only one of the four different surface sites, because at that site only the modified group does not disturb the regular pattern of interactions at the crystal surface. Occlusion of such a molecule through the (011) face will lead to a crystal sector of symmetry $P 1$, since the occluded molecules therein are related by only translation symmetry. Occlusion of additive through all four diagonal faces $\{011\}$ will lead to a crystal composed 
of four sectors, where these four sectors are related to each other by the $2 / m$ point symmetry of the original host crystal.

Consequently, in general, an additive molecule will be anisotropically distributed within the grown crystal, preferentially occluded through different subsets at surface sites on the various faces, leading to a mixed crystal composed of sectors coherently intertwined. The selective occlusion of the additive into a sector will thus lead to a reduction of the crystal symmetry to the symmetry of the face through which it was adsorbed. This principle holds for each crystal sector, although the occluded additive may occupy only a small fraction of the bulk sites. It also holds for the whole crystal, but we note that the different crystal sectors are related to each other by the symmetry elements of the point group of the pure crystal structure. The reduced symmetry of each sector may thus be masked, depending upon the history of crystal growth, whether the sectors are examined separately, and the type of examination. For example, in a crystal containing all sectors, the loss of a glide plane or twofold screw axis should be immediately evident from the lack of the corresponding systematic absences in the X-ray or neutron diffraction pattern (naturally assuming that the crystal is of mosaic texture, which is the general case, and so the different mosaic blocks

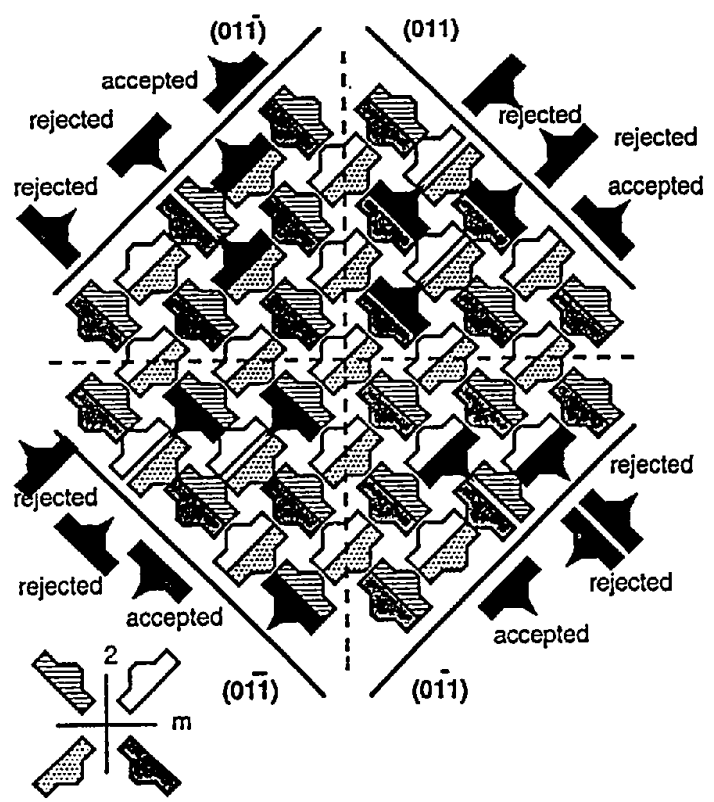

Fig. 12. Schematic representation of adsorption and occlusion of additive molecules (in black) through the four slanted $\{011\}$ faces in a centrosymmetric crystal space group $P 2_{1} / c$, containing four symmetryrelated molecules. Their relative orientations are also depicted in the insert (point symmetry $2 / m$ ). In each of the four crystal sectors, only one of the symmetry-related molecules can be replaced by the additive on adsorption, which means the loss of all symmetry elements but translation. The crystal symmetry in each sector is thus reduced from $P 2_{1} / c$ to $P$ l. diffract independently), but not the loss of a rotation axis, mirror plane or center of inversion. Detection of the latter would require at least measurement of the complete set of diffraction data from the individual sector to test for a reduction in Laue diffraction symmetry, generally to be followed by crystal structure refinement. Reduction in crystal symmetry following the above principles was predicted in several host-additive systems and experimentally demonstrated by a variety of methods including changes in crystal morphology, HPLC, frequency doubling of laser light (i.e. second harmonic generation SHG), solid-state asymmetric photodimerization and optical birefringence. Symmetry-lowering has also been directly shown by X-ray and neutron diffraction. We now review examples to illustrate the arguments and principles described above.

\subsection{Detection of reduced crystal symmetry by enantio- meric distribution of additives}

The mode of occlusion of additive leading to a reduction in symmetry, depicted schematically in Fig. 12, may be used as an aid to understand the selective occlusion of $R, S$-threonine into the crystals of $(R, S)$-serine (Weissbuch, Shimon et al., 1985). The morphological changes induced in $(R, S)$-serine, as discussed in the previous section (see Fig. 10), imply that $R$-threonine, if occluded, would do so through the $(011)$ and $(01 \overline{1})$ faces. By symmetry, $S$ threonine would be occluded through the $(011)$ and $(0 \overline{1} \overline{1})$ faces. Thus, the threonine additives, racemic in

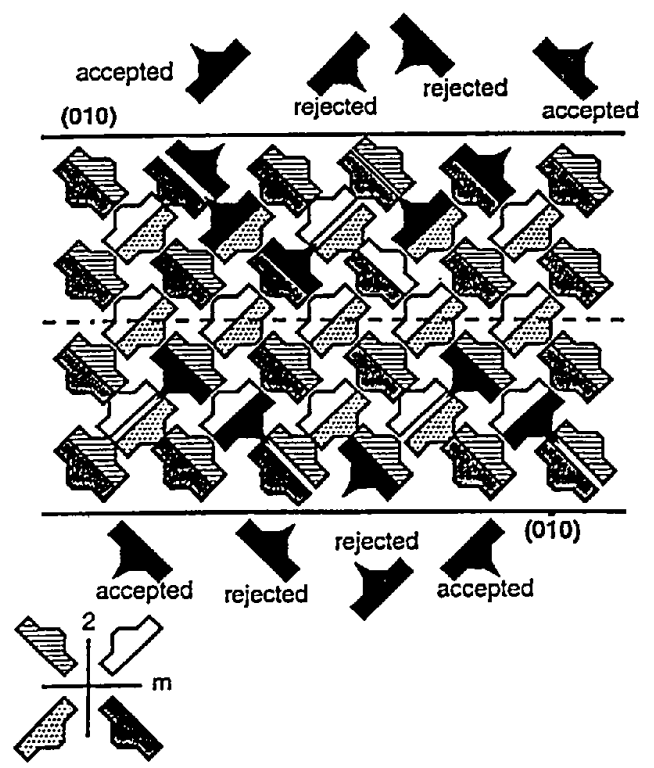

Fig. 13. Schematic representation of adsorption and occlusion of additive molecules (in black) through the top and bottom $\{010\}$ faces in a crystal of space group $P 2 / c$, resulting in the loss of the glide and inversion symmetry. The crystal symmetry in each half is thus reduced from $P 2_{1} / c$ to $P 2_{1}$. 
solution, must on occlusion into $(R, S)$-serine crystals be enantiomerically segregated into domains of opposite chirality along the $b$-axis during crystal growth, as was indeed experimentally shown by HPLC methods (Weissbuch, Shimon et al., 1985).

Symmetry lowering of a somewhat similar nature involved the $\alpha$-form of glycine grown in the presence of $\alpha$ amino acid additives (Weissbuch et al., 1983). As an aid to understanding we present Fig. 13, which is a model representation of reduction in symmetry from $P 2_{1} / c$ to $P 2_{1}$ when molecules are occluded through the $(010)$ and ( $0 \overline{1} 0)$ faces. The mode of adsorption of $\alpha$-amino acid additives onto the $\{010\}$ faces of $\alpha$-glycine has already been described in $\$ 3$. In terms of that analysis, only the $R$ - $\alpha$-amino acid additive may be occluded through the (010) face, replacing a glycine molecule at sites (1) and (2) only [Fig. 3(a)], yielding a pyramidal morphology [Fig. 3(c)]. By symmetry, only the $S$ - $\alpha$-amino acid additives may be occluded into sites (3) and (4) through the $(0 \overline{1} 0)$ face of $\alpha$-glycine [Fig. 3(a)], yielding an enantiomorphous morphology [Fig. 3(d)]. Thus, racemic additives cause the formation of $\{010\}$ plates [Fig. 3(e)], because growth at the $+b$ and $-b$ sides of the crystal is inhibited. These plates were found to contain $0.02-0.2 \%$ racemic $\alpha$-amino acid additive within the crystal bulk with the two enantiomers totally segregated in the two crystal sectors at the $+b$ and $-b$ halves As expected, the $R$-enantiomers populate the $+b$, and the $S$-enantiomers the $-b$ half of the crystal according to HPLC measurements (Weissbuch et al., 1983). In terms of the argument given above, the crystal symmetry of each half of the crystal must be reduced from $P 2_{1} / n$ to $P 2_{1}$, and the two sectors are enantiomorphous. We note that the absolute configuration of the $\alpha$-amino acid additive can be directly assigned not only from the induced changes in crystal morphology but also from its anisotropic distribution on occlusion within the crystal. Symmetry lowering of a similar nature was also demonstrated in another system, which involved enantiomeric segregation of the dipeptide glycyl-leucine inside growing crystals of glycyl-glycine ${ }^{+} \mathrm{H}_{3} \mathrm{NCH}_{2} \mathrm{CONHCH}_{2} \mathrm{CO}_{2}^{-}$ (Weissbuch, Berkovitch-Yellin, Leiserowitz \& Lahav, 1985).

\subsection{Detection of reduced crystal symmetry by non- linear optics}

Symmetry lowering in host-additive systems, such as a loss of inversion, glide or twofold screw symmetry, which would introduce (additional) polarity into the crystal, may be probed by nonlinear optical effects, such as second harmonic generation (SHG) which is active in a noncentrosymmetric material. One requirement is that either the host or additive molecule has a large molecular hyperpolarizability tensor $\beta$, leading to large optical nonlinearity. Consequently, a centrosymmetric pure crystal structure, which consists of antipar-
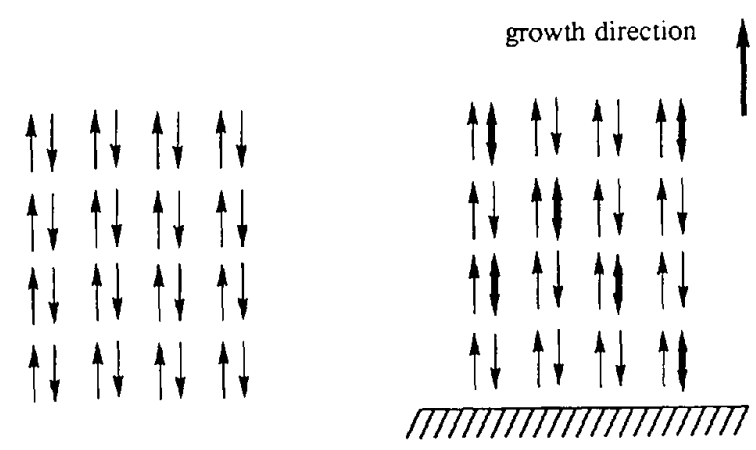

blocked direction pure

(a) grown in the presence of a centrosymmetric additive

(b)
Scheme 5.

allel pairs of host molecules with high $\beta$ coefficients [Scheme $5(a)$ ], would yield a SHG-active crystal upon site-selective occlusion by a centrosymmetric guest molecule, as shown in Scheme 5(b). Because the growth of the crystal is blocked at the bottom face, the additive is adsorbed and occluded through only one of the pair of surface sites at the top exposed face. We demonstrated the potential of this approach with centrosymmetric host crystals of $p$-( $N$-dimethylamino) benzylidene$p^{\prime}$-nitroaniline [ $3 a$ (Fig. 14)], which became noncentrosymmetric and SHG-active upon site-selective occlusion of the guest molecule $p, p^{\prime}$-dinitrobenzylideneaniline ( $3 b$ ), which is pseudocentrosymmetic (Weissbuch, Lahav, Leiserowitz, Meredith \& Vanherzeele, 1989). In this system the host and guest molecules have large and negligible $\beta$-coefficients, respectively.<smiles>CN(C)c1ccc(/C=N/c2ccc([N+](=O)[O-])cc2)cc1</smiles><smiles>O=[N+]([O-])c1ccc(/C=N/c2ccc([N+](=O)[O-])cc2)cc1</smiles>

(3b)

The reverse situation, where the guest has large hyperpolarizability, is illustated by an $\alpha$-glycine host crystal containing, as additive, $\alpha$-amino acids which have a high $\beta$ coefficient, such as the $p$-nitrophenyl derivatives of $\alpha, \delta$-diamino butyric acid $(4 a)$, ornithine $(4 b)$ and lysine $(4 c)$. The amount of occluded additive was less than $0.2 \%$ ( $w t / w t$ of glycine), but sufficient to yield colored crystals. In terms of the arguments presented above, $\{010\}$ plate-like crystals of glycine, grown in the presence of $R, S$-additives, should have their symmetry reduced from $P 2_{1} / n$ to $P 2_{1}$. In fact, the symmetry was 


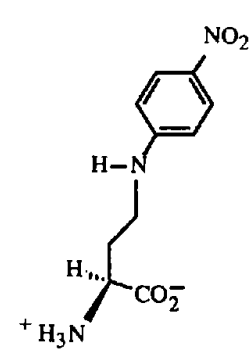

(4a)

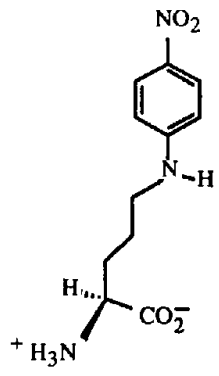

(4b)

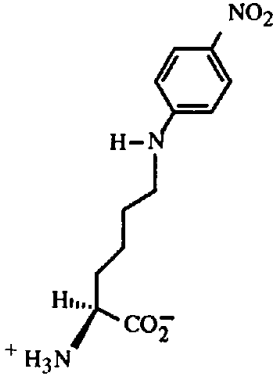

$(4 c)$ reduced even further to $P 1$ according to the SHG measurements (Weissbuch, Lahav, Leiserowitz, Meredith \& Vanherzeele, 1989), providing additional information on the nature of the crystal growth process.

Other systems of interest are the noncentrosymmetric orthorhombic crystals of space-group symmetry $P 2{ }_{1} 2{ }_{1} 2_{1}$. On symmetry grounds such crystals are nonpolar along the three principal crystallographic axes and therefore frequency doubling is forbidden in these directions. We anticipated that such crystals, when grown in the presence of appropriate additives so as to reduce the crystal symmetry to monoclinic $P 2_{1}$, should become SHGactive along the remaining $2_{1}$ axis but not along those two axes which have lost their twofold screw symmetry. In fact, single crystals of $(S)$-glutamic acid. $\mathrm{HCl}$ grown in the presence of appropriate additives were SHG-active

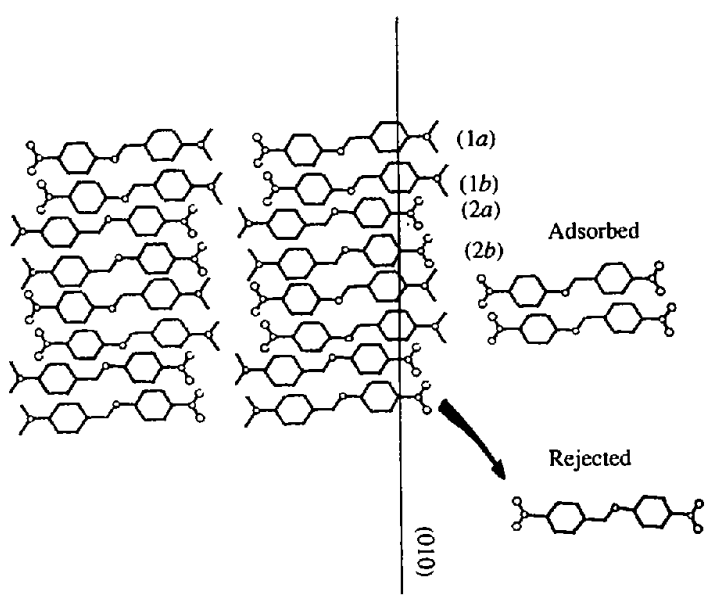

(a)
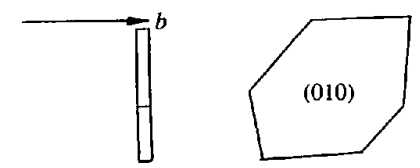

(b)

Fig. 14. (a) Packing arrangement of a polymorph of the $p$-( $N$-dimethylamino)benzylidene- $p^{\prime}$-nitroaniline crystal, showing how the dinitro additive may be adsorbed on the $(010)$ face. The additive may be adsorbed only at sites of type (1), but not at type (2) sites. (b) Two views of the morphology of the plate-like crystals. along all three axes (Weissbuch, Lahav, Leiserowitz, Meredith \& Vanherzeele, 1989), indicating symmetry lowering to $P 1$, once again providing information on the nature of the crystal growth process.

\subsection{Detection of reduced crystal symmetry by optical birefringence}

The properties of optical birefringence in crystals have been used to demonstrate a reduction in crystal class. McBride \& Bertman (1989) took advantage of the fact that crystals that belong to a high symmetry class, such as tetragonal, trigonal or hexagonal, are optically uniaxial, whereas crystals that belong to a lower crystal class, such as triclinic, monoclinic or orthorhombic, are optically biaxial. They studied the tetragonal crystals of di(11-bromoundecanoyl) peroxide (5a), denoted as $\mathrm{Br} \cdots \mathrm{Br}$, in the presence of guest $(5 b)$ where $\mathrm{a} \mathrm{Br}$ atom is replaced by $\mathrm{CH}_{3}$, denoted as $\mathrm{Br} \cdots \mathrm{CH}_{3}$. In the host crystal $\mathrm{Br} \cdots \mathrm{Br}$ molecules assemble into layers which have $\mathrm{Br}$ atoms on both the upper and lower surfaces.

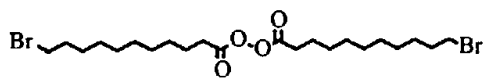<smiles>CCCCCCCCCC(=O)OOC(=O)CCCCCCCCBr</smiles>

(5b)

Successive layers stack $\mathrm{Br}$ to $\mathrm{Br}$ atoms with a $90^{\circ}$ rotation about a fourfold screw axis in the stacking direction. These crystals, which grow as square \{001\} plates delineated by four $\{110\}$ side faces, are not birefringent for light travelling along the fourfold screw axis perpendicular to the plate, appearing dark between crossed polarized filters. Crystals of $\mathrm{Br} \cdot \cdots \mathrm{Br}$ containing $15 \% \mathrm{Br} \cdot \mathrm{CH}_{3}$ are birefringent, the plate revealing four sectors under crossed polarizers. Birefringence in each sector results from unsymmetrical incorporation of the $\mathrm{Br} \cdots \mathrm{CH}_{3}$ additives during crystal growth. As McBride states in his paper, this method of optical microscopy is convenient for surveying large numbers of crystals, to catalogue their growth patterns, and to identify reduced symmetry domains that may be characterized by diffraction. Such an approach has been followed up by Gopalan, Peterson, Crundwell \& Kahr (1993) who have observed reduction in symmetry both by optical birefringence and $\mathrm{X}$-ray crystallographic studies of different sectors in mixed crystals of $\mathrm{NaBrO}_{3} / \mathrm{NaClO}_{3}$ and $\mathrm{NaNO}_{3} / \mathrm{Pb}\left(\mathrm{NO}_{3}\right)_{2}$. Garnets where $\mathrm{Al}^{3+}$ were replaced by $\mathrm{Fe}^{3+}$ ions also show a reduction in lattice symmetry from cubic down to triclinic (Allen \& Buseck, 1988).

\subsection{Probing intermolecular forces through reduced crystal symmetry}

There is interest in investigating the minimal modification of the tailor-made additive with respect to 
the host molecule, that would still be recognized and discriminated by the growing crystal surface, and still lead to a reduction in symmetry. Indeed, solid solutions composed of host and additive molecules of similar structure and shape have generally been believed to exhibit the same symmetries as those of the host crystals. A reduction in crystal symmetry involving a structure determination by X-ray or neutron diffraction should allow one to probe fine interactions between host and additive at the crystal surfaces.

We first discuss solid solutions of carboxylic acids $\left(\mathrm{XCO}_{2} \mathrm{H}\right)$ in primary amides $\left(X \mathrm{CONH}_{2}\right)$, where an $\mathrm{NH}_{2}$ group is substituted by an $\mathrm{OH}$ moiety (Scheme 6). A strong inhibition of growth develops along the direction of the $\mathrm{O}=\mathrm{C}-\mathrm{N}-\mathrm{H}_{a} \cdots \mathrm{O}=\mathrm{C}$ hydrogen bond, $\mathrm{H}_{a}$ being the amide $\mathrm{H}$ atom in an antiperiplanar conformation to the $\mathrm{C}=\mathrm{O}$ system. Inhibition arises when a carboxylic acid additive attempts to force the antiperiplanar lonepair electron lobes of its $\mathrm{OH}$ group against a carbonyl oxygen of the underlying molecule, an atom that would normally form a hydrogen bond with the amide's antiperiplanar hydrogen. Incorporation of a carboxylic acid in this orientation would substitute a $8 \mathrm{kj} \mathrm{mol}^{-1}$ repulsion for a $25 \mathrm{kj} \mathrm{mol}^{-1}$ attraction. The carboxylic acid additive molecule will thus avoid surface sites that require the $\mathrm{OH}$ groups to be oriented towards the surface and be preferentially adsorbed at sites where the $\mathrm{OH}$ group emerges from the surface (Berkovitch-Yellin et al., 1985).

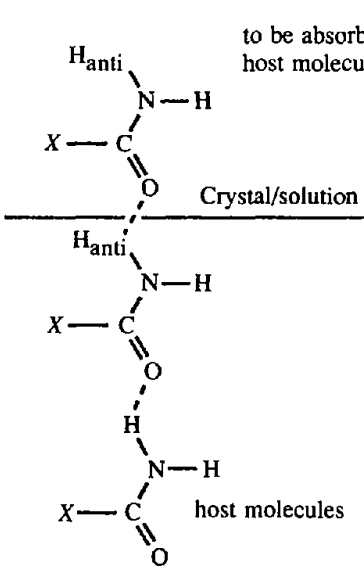

(a) dimerization method was applied to the host:additive system $E$-cinnamamide:E-cinnamic acid (Vaida et al., 1989) where small amounts $(<2 \%)$ of cinnamic acid guest were occluded into the host crystal.

$(S)$-Asparagine, $\mathrm{H}_{2} \mathrm{NOCCH}_{2} \mathrm{CH}\left(\mathrm{NH}_{3}^{+}\right) \mathrm{CO}_{2}^{-}$, crystallizes from water as a monohydrate with a tight threedimensional net of hydrogen bonds in a $P 2_{1} 2_{1} 2_{1}$ structure [Fig. 15(a)]. The morphology is prismatic, with 18 developed faces [Fig. 15(b), top]. Crystallization of $(S)$-asparagine in the presence of $S$-aspartic acid, $\mathrm{HO}_{2} \mathrm{CCH}_{2} \mathrm{CH}\left(\mathrm{NH}_{3}^{+}\right) \mathrm{CO}_{2}^{-}$, yields $\{010\}$ plates [Fig. $15(b)$, bottom]. In terms of the arguments already given for amide-acid systems, the guest aspartic acid molecule should be more easily adsorbed at sites (1) and (3), on the growing (010) surface, than at sites (2) and (4) [Fig. 15(c)]; the reverse situation holds for the opposite $(0 \overline{10})$ face. If, on growth of the mixed crystal, the $(0 \overline{1} 0)$ face is blocked so that the amide and acid molecules would only be occluded through the (010) face, the symmetry of the mixed crystal should be reduced to $P 12_{1} 1$. A low-temperature $(18 \mathrm{~K})$ neutron diffraction study with deuterated aspartic acid in protonated asparagine showed the expected reduction in symmetry (Weissinger-Lewin et al., 1989).

Analogously, additive $E$-cinnamic acid, $\mathrm{C}_{6} \mathrm{H}_{5}-\mathrm{CH}=$ $\mathrm{CH}-\mathrm{CO}_{2} \mathrm{H}$, induces a loss of the center of inversion in the crystal of $E$-cinnamamide, $\mathrm{C}_{6} \mathrm{H}_{5}-\mathrm{CH}=\mathrm{CH}-$

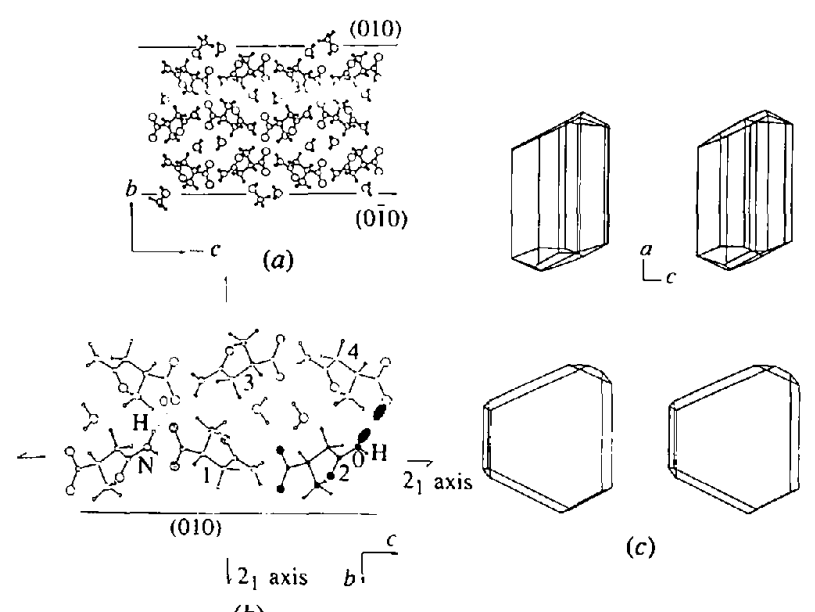

(b)

Fig. 15. (a) Packing arrangement of ( $S$ )-asparagine monohydrate viewed along the $a$-axis. Four adsorbed methanol molecules are inserted on the two $\{010\}$ faces, referring to crystals grown from water-methanol solution. (b) Preferential adsorption of aspartic acid on the (010) surface of asparagine (open circles) at sites of type 1 and 3 rather than 2 and 4 , as the crystal is growing at the (010) face. Molecules at sites 1 and 3 are shaded, indicating the latter may be occupied by either asparagine or aspartic acid. The probability for aspartic acid to be adsorbed at site 2 (full circles) is less than at site 1 because of $O$ (hydroxyl) . . O (carboxylate) lone-pair electron repulsion between 2 and 4. (c) Stereoscopic view of morphology of (S)-asparagine monohydrate: pure crystals grown from aqueous solution (top), grown in the presence of $(S)$-aspartic acid (bottom), or in water-methanol solution. 
$\mathrm{CONH}_{2}$, which in pure form appears in a centrosymmetric monoclinic arrangement, space group $P 21 / c$. The crystal structure [Fig. 16(a)] is composed of hydrogenbonded dimers interlinked by $\mathrm{N}-\mathrm{H} \cdots \mathrm{O}$ bonds to form a ribbon-like motif which runs parallel to the $b$-axis. $E$-cinnamic acid is preferentially occluded through half the $\{011\}$ surface sites of the crystal at opposite ends of these ribbons, resulting in a crystal [Fig. 16(b)] with two enantiomorphous halves of at most $P 2_{1}$ symmetry. This reduced symmetry was proven photochemically (Vaida et al., 1989). UV irradiation of $E$-cinnamamide yields centrosymmetric photodimers, by virtue of a cyclobutane ring formation involving pairs of close-packed $>C=C<$ bonds across centers of inversion (Fig. 17). Replacement of one such pair by $E$-cinnamic acid results in the formation of asymmetric cinnamamide-cinnamic acid photodimers of opposite chirality at the two enantiomorphous halves of the mixed crystal, with an enantiomeric ratio of 60:40 at each opposite half.

Reduction in crystal symmetry was demonstrated by photodimerization, $X$-ray and neutron diffraction for the host-guest crystal structure of $E$-cinnamamide-2$E$-thienylacrylamide (Vaida et al., 1988; Shimon et al., 1993). The host structure [Fig. 16(a)] incorporates herringbone contacts between aromatic $\mathbf{C}-\mathbf{H}$ groups and $\pi$-electron clouds of neighboring phenyl rings. In order

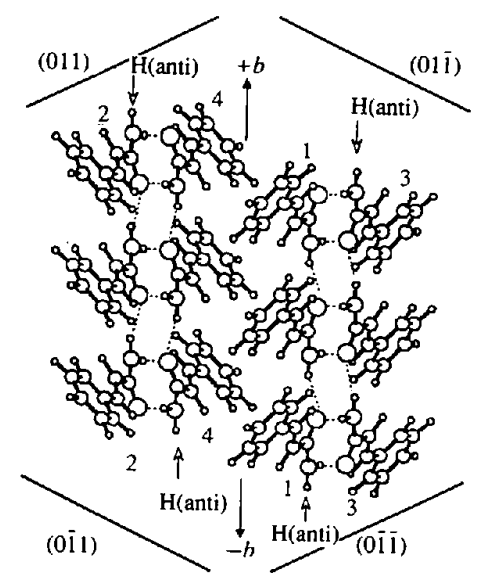

(a)

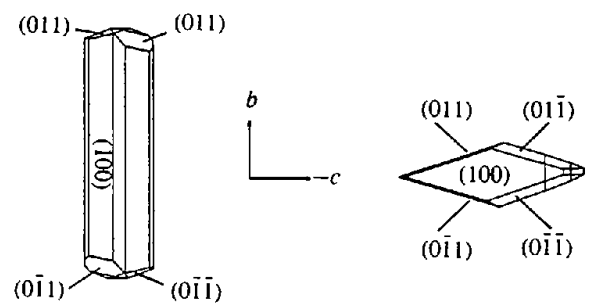

(b)

Fig. 16. (a) Packing arrangement of $E$-cinnamamide viewed along the $a$ axis delineated by the $\{011\}$ faces. (b) Morphology of $E$-cinnamamide crystals: pure (left) and grown in the presence of cinnamic acid (right). to induce site-selective adsorption and occlusion, these contacts are replaced in the host-guest system by unfavorable contacts between sulfur lone-pair electrons and the $\pi$-electron system [Fig. 18(a)]. Thienylacrylamide is occluded through the enantiotopic $\{011\}$ faces of

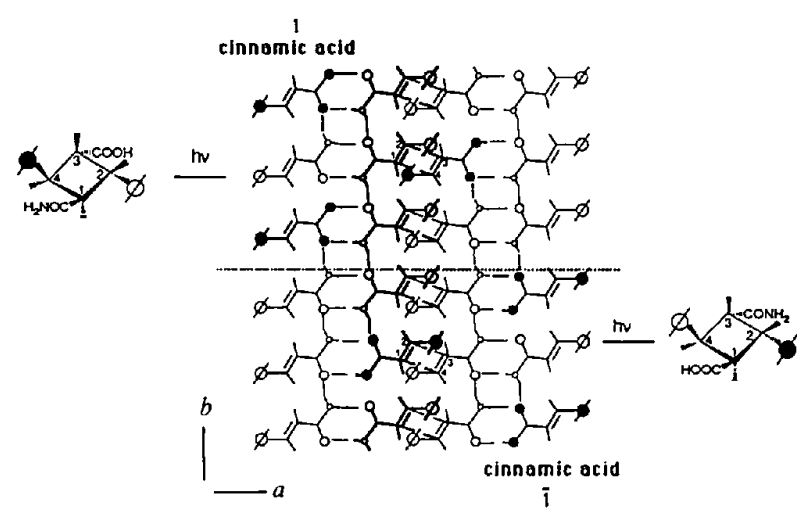

Fig. 17. Ribbons along the $b$-axis of hydrogen-bonded molecules composed of $E$-cinnamamide (open circles) and occluded guest $E$-cinnamic acid molecules (full circles). The latter were adsorbed through site (1) at the $+b$ end and site (i) at the $-b$ end, leading to two enantiomorphous domains joined at the central dotted line. Also shown are the ribbons related by translation along the $a$-axis, thus depicting the close $4 \AA$ contact between $C=C$ double bonds related by a center of inversion. Two enantiomorphous cyclobutane photodimers between the host and occluded guest from the top and bottom halves of the crystal are also shown.

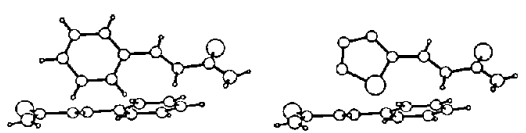

(a)
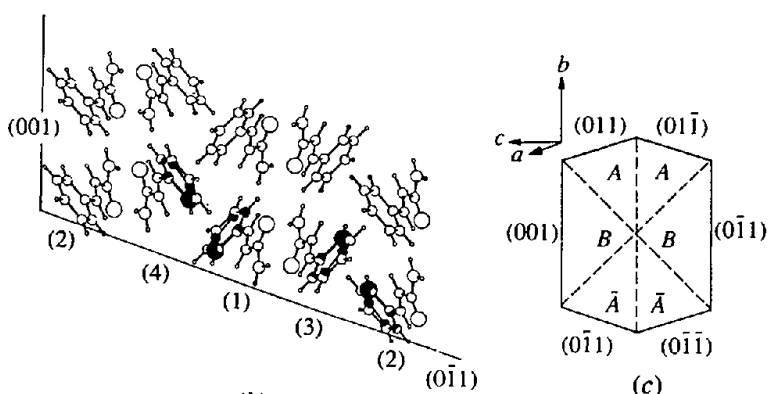

(c)

Fig. 18. (a) Herringbone contacts involving: (left) two cinnamamide molecules, (right) host cinnamamide and guest thienylacrylamide molecules. (b) The four different surfaces sites (1), (2), (3) and (4) of $E$-cinnamamide at the $(0 \overline{1} 1)$ face. $S$ and $C$ atoms of the superimposed 2-thienyl rings in the positions they would assume were they to replace the phenyl rings of cinnamamide molecules at the $(0 \overline{1} 1)$ face are shaded. Site (1) is preferred for guest adsorption on the (0ī1) face since the $S$ atom at that site emerges from the crystal face. By the same argument, (1) and (2) are the preferred sites for guest adsorption on the (001) face. (c) Morphological representation of the cinnamamide-thienylacrylamide crystal with six sectors of reduced symmetry. 
the crystal [Fig. 18(b)]. These faces exhibit $p 1$ plane symmetry, since the four different surface sites are not related by symmetry. The guest can easily be adsorbed at only one of these four sites at which the sulfur emerges from the face, leading to $P 1$ crystal symmetry upon occlusion of the guest. Thienylacrylamide is also occluded through the $\{001\}$ faces, which exhibit $p m$ plane symmetry. Occlusion through faces of this type should lead to crystal symmetry $P c$. The mixed crystal should be thus divided into six sectors of reduced symmetry [Fig. 18(c)]. The different sectors of the same type, such as $A$ and $\bar{A}$ are related to one another by the $2 / m$ point symmetry of the host crystal. Low-temperature $\mathrm{X}$-ray and neutron diffraction studies on $A$ - and $\bar{A}$-type sectors, cut from a crystal specimen showed symmetry $P 1$, in keeping with the photodimerization studies. The proposed reduction in symmetry of the $B$-type sector [Fig. 18(c)] was proven to be correct, but only by an Xray diffraction analysis. We may infer from these results a repulsive intermolecular interaction between the sulfur lone-pair electrons and electron system of neighboring molecules, but we do not have an estimate of the energy.

We have elucidated, by changes in crystal morphology, reduction in crystal symmetry and energy calculations, the molecular conformation of a flexible additive and the effect of diastereomeric chiral additives on crystal morphology and symmetry. For example, for the centrosymmetric crystal of the dipeptide glycyl-glycine when grown in the presence of glycyl-leucine additive, the latter adopts a specific conformation which minimizes unfavorable additive-host molecular interactions at the site of adsorption (Weissbuch, Berkovitch-Yellin, Leiserowitz \& Lahav, 1985; Berkovitch-Yellin, Weissbuch \& Leiserowitz, 1989).

The pronounced effect threonine exerts on the morphology and structure of racemic serine crystals has been discussed in $\$ 4.2$ and $\$ 5.1$. Oddly enough, additive allothreonine, the diastereoisomer of threonine, does not alter very much the morphology of racemic serine crystals [Fig. 11(e)], nor is there any enantioselective segregation of allothreonine along the $b$-axis of the crystal. These observations were accounted for in a quantitative way by calculated surface binding energies, according to which, say $S$-allothreonine, is most strongly bound on the $\{100\}$ faces and somewhat less so on the (011) and $(01 \overline{1})$ faces of racemic serine crystals. We note that $R$ - and $S$-allothreonine additives are equally well occluded through the $\{100\}$ faces because of the $2 / m$ crystal point symmetry of $(R, S)$-serine, thus masking any enantioselective segregation of the additive through the $\{011\}$ faces (Weissbuch, Shimon et al., 1985).

\subsection{Some general rules on symmetry lowering through additive occlusion}

As a conclusion of this section on crystal symmetry lowering, we may draw some general rules. The local symmetry of a mixed crystal is determined by that of the faces developed. If the symmetry of the crystal face through which an additive is adsorbed is not lower than that of the bulk, there cannot be reduction in symmetry for the crystal sector bound by that face. In other words, the symmetry of a crystal sector cannot be lower than that of the growth sites on the bounding crystal face. This principle is so basic that if a crystal is bound by faces of different plane symmetries, one can, at least in principle, imagine it as formed of different sectors each with bulk symmetry as low as that of its bounding face, the sectors being related to each other by the point symmetry elements of the crystal. Thus, the 'overall' symmetry of the crystal is not reduced. This is a consequence of Curie's principle, as presented in his paper 'Symetrie dans les phenomenes physiques' (Curie, 1894): a physical event cannot have a symmetry lower than that of the event which caused it.

\section{Effect of tailor-made additives on crystal texture}

Surprisingly, little work has been done to date on the effect of tailor-made additives on mosaicity and coherence length (i.e. extent of perfect crystal order) in molecular crystals. Indirect evidence was first brought to light by low-temperature $(18 \mathrm{~K})$ neutron diffraction measurements on single crystals of pure asparagine monohydrate and the solid solution of asparagine-aspartic acid monohydrate (WeissingerLewin et al., 1989), undertaken to demonstrate symmetry lowering in the latter system (\$5.4). The neutron reflections of the specimen crystal of pure asparagine suffered from pronounced extinction effects, whereas the specimen crystal of asparagine-aspartic acid was almost free from extinction. These results were interpreted in terms of much higher mosaicity and smaller mosaic domains in the asparagine-aspartic acid crystals, brought about by the presence of aspartic acid. More direct evidence came from studies on biomineralization (Berman et al., 1990, 1993a,b), which addressed the fundamental question of whether proteins in calcite mineralized tissue alter the crystal texture in an anisotropic manner. High-resolution synchrotron X-ray diffraction was used to monitor changes in coherence length and angular mosaic spread. It was found that protein extracted from sea urchins and mollusks reduced the coherence lengths of crystals of calcium fumarate and calcium malonate in the directions perpendicular to the planes onto which the proteins were preferentially adsorbed. In contrast, the calcite crystals grown in vitro in the presence of the proteins exhibited an increase in angular spread, but no anisotropic effect in coherence length was detected. A biologically produced calcite crystal, on the other hand, showed a preferential reduction in coherence length in the direction of the $c$-axis. 


\section{Effect of solvent on crystal growth and morphology}

Solvent has a strong influence on the habit of crystalline materials, however, the role played by solvent-surface interactions in enhancing or inhibiting crystal growth is still not completely resolved. To date there have been two different approaches to clarify this point. In one theory, proposed by Bennema and others (Bennema \& Gilmer, 1973; Bennema, 1992; Bourne \& Davey, 1976; Elwenspoek, Bennema \& van Eerden, 1987), favorable interactions between solute and solvent on specific faces leads to reduced interfacial tension, causing a transition from a smooth to a rough interface and a concomitant faster surface growth. Alternatively, it has been proposed that preferential adsorption at specific faces will inhibit their growth as removal of bound solvent poses an additional energy barrier for continued growth. Our studies on the role played by tailor-made additives are in keeping with the latter approach. However, solvent-surface interactions cannot be as clearly analyzed as those between the tailor-made additives and the crystal. In order to help clarify this problem, we describe several approaches that have been adopted.

\subsection{Computational methods}

One of the main difficulties is to disentangle the relative contributions of the internal crystal structure and solvent-surface interactions in determining the crystal morphology. The former may be evaluated by growing the crystal by sublimation, if possible, or by theoretical modeling. The ground rules for a quantitative determination of crystal morphology were laid down by Hartman \& Perdok (1955a,b), Hartman (1973) and Hartman \& Bennema (1980). According to their analyses, the crucial relation is between the layer energy, $E_{L}$, which is the energy released when a layer is formed, and the attachment energy, $E_{\text {att }}$, which is defined as the energy per molecule released when a new layer is attached to the crystal face. $E_{\text {att }}$ controls the growth rate perpendicular to the layer, while $E_{L}$ measures the stability of the layer. The working hypothesis is that the morphological importance of a crystal face is inversely proportional to its attachment energy. The energies, $E_{L}$ and $E_{\text {att }}$, may be computed using atom-atom potential energy functions, which are generally made up of van der Waals and electrostatic terms. The latter term is described by an interatomic Coulombic interaction with parameters which depend upon charge distribution and so may be derived by quantum chemical methods, self-consistent force field methods, or from experimental deformation electron-density distributions as determined from lowtemperature X-ray diffraction data (Hirshfeld, 1977). By applying such a procedure it became possible to calculate the energy values of $E_{L}$ and $E_{\text {att }}$ for various low-index crystal faces from which the 'theoretical crystal form' may be derived. This method has been applied for a variety of organic (e.g. Berkovitch-Yellin,
1985; Berkovitch-Yellin et al., 1985; Boek, Feil, Briels \& Bennema, 1991; Docherty, Roberts, Saunders, Black \& Davey, 1993) and inorganic (Woensdregt, 1993) crystals.

It is possible to use such information on the theoretical form to help understand the effect of solvent on crystal morphology. For example, the theoretical form of $\alpha$-glycine (Berkovitch-Yellin, 1985; Boek, Feil, Briels \& Bennema, 1991) calculated by this method [Fig. $19(a)]$ is in good agreement with the morphology of $\alpha$-glycine crystals obtained by sublimation [Fig. $19(b)$ ]. This morphology exhibits well developed $\{010\}$ faces, in keeping with the dominant hydrogen-bonding energy of the molecular bilayer in the $a c$-plane. However, glycine crystals grown from aqueous solution display a distinctly different morphology, which is bipyramidal with large $\{011\}$ and $\{110\}$ faces and less well developed $\{010\}$ faces [Fig. 3(b)]. In order to understand these differences, substrate-solvent interactions were taken into account. The large $\{011\}$ and $\{110\}$ faces expose $\mathrm{CO}_{2}^{-}$and $\mathrm{NH}_{3}^{+}$groups well oriented to have a strong affinity for water. The $\{010\}$ face exposes alternating layers of $\mathrm{C}-\mathrm{H}$ or $\mathrm{CO}_{2}^{-}$and $\mathrm{NH}_{3}^{+}$groups [Fig. 3(a)], and so, on average, is less hydrophilic than the $\{011\}$ and $\{110\}$ faces. Moreover, if the solute glycine molecules dock into surface sites primarily as cyclic hydrogenbonded dimers, the $\{010\}$ face will essentially expose $\mathrm{C}-\mathrm{H}$ groups. These observations were expressed by Berkovitch-Yellin in a semi-quantitative form by calculating the Coulomb potential at the various faces, which indicated preferential adsorption of water molecules onto the polar faces. Further support for such arguments comes from the observed crystal morphology of the $\alpha$-amino acids with hydrophobic chains such as phenylalanine (Weissbuch, Frolow, Addadi, Lahav \& Leiserowitz, 1990), valine and isoleucine (Torii \& Yitaka, 1970, 1971). Their crystal structures are composed of hydrogen-bonded bilayers as in $\alpha$-glycine, and their morphologies are plate-like when grown from aqueous solution. Clearly the hydrophobic chains provide in-

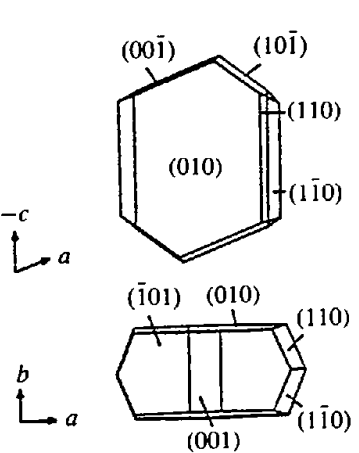

(a)

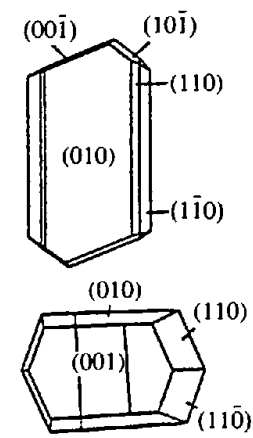

(b)
Fig. 19. (a) Theoretical crystal form of $\alpha$-glycine. (b) Morphology of $\alpha$-glycine crystals obtained by sublimation. 
creased layer energy and a lower binding concentration of water at the sides of such bilayers than in $\alpha$-glycine, providing a strong propensity for plate formation.

\subsection{Crystalline solvates}

Another approach involved the use of crystalline solvates, where the solvent of crystallization plays the dual role of solvent and crystal solute. Such crystals were grown in the added presence of tailor-made solvent, which is a slightly modified version of the solvate solvent. It was found when crystalline hydrates were grown from aqueous solution in the added presence of methanol (the 'tailor-made' solvent), that the change in morphology was interpretable (Shimon, Vaida, Addadi, Lahav \& Leiserowitz, 1990, 1991) in a manner akin to the effect of a tailor-made inhibitor. This approach is illustrated with the crystal of $\alpha$-rhamnose monohydrate, which contains a polar arrangement [Fig. 20(a)] in space group $P 2_{1}$ and, when grown from pure aqueous solution, displays a bipyramidal morphology [Fig. 20(b)]. The two $\mathrm{O}-\mathrm{H}$ bonds of the hydrate water molecules are oriented towards the $+b$ direction, but not towards the $-b$ direction of the crystal. Thus, addition of methanol as a cosolvent changes the morphology of the crystal, completely inhibiting growth along the $+b$ direction and yielding a pyramidal crystal [Fig. 20(c)].

A second example is $(S)$-asparagine. $\mathrm{H}_{2} \mathrm{O}$. Crystals grown from pure water are delineated by 18 faces [Fig. 15(b), top]. The packing arrangement (Fig. 15a), shows that one of the two $\mathrm{O}-\mathrm{H}$ bonds of each water

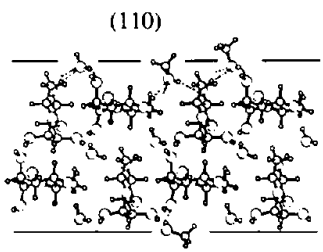

(iijo)

(a)

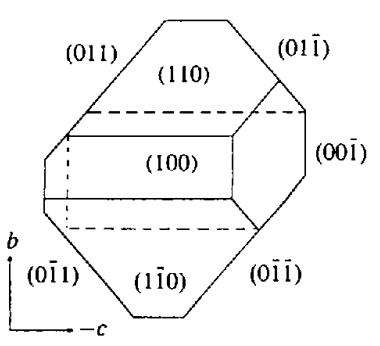

(b)

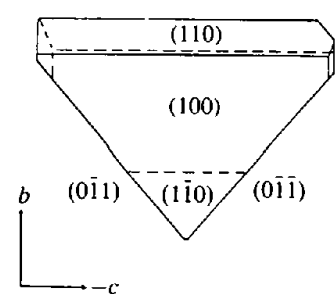

(c)
Fig. 20. (a) Packing arrangement of $\alpha$-thamnose monohydrate crystal viewed along the $a$-axis; the $\mathrm{OH}$ bonds of the hydrate water molecules point towards the $+b$, but not the $-b$ direction; replacement of water by methanol on the $\{110\}$ faces is depicted. $(b)-(c)$ Crystals viewed along the $a$-axis. Morphologies of $\alpha$-rhamnose monohydrate crystals grown from: (b) aqueous solution and (c) 9:1 methanol:water solution. molecule emerges from the $\{010\}$ faces, which are poorly expressed. Therefore, methanol molecules can be easily attached at these surface sites [see Fig. 15(a)]. The methyl group of each adsorbed methanol protrudes from the two $\{010\}$ faces, impedes growth in the $b$ direction and thus induces the formation of large $\{010\}$ crystal faces [Fig. 15(b), bottom]. On the $\{011\}$ faces, only two of the four symmetry-related molecules can be substituted by methanol and so inhibition of $\{011\}$ should be less dramatic. This reasoning proved to be in keeping with experiment (Shimon, Vaida, Addadi, Lahav \& Leiserowitz, 1991); growth in the presence of methanol as additive yielded $\{010\}$ plates similar in shape to when asparagine is grown in the presence of aspartic acid [Fig. 15(b)]. That methanol can indeed be selectively adsorbed on both the $\{010\}$ and $\{011\}$ faces was further demonstrated by experiments involving partial dissolution of asparagine. $\mathrm{H}_{2} \mathrm{O}$ crystals in methanol solution, revealing etch-pits, although poorly developed, on only these faces (Shimon, Lahav \& Leiserowitz, 1986).

\subsection{Polar crystals}

In another approach, use was made of the principle that in a polar crystal the attachment energy $E_{\text {att }}$ at opposite and hemihedral faces $(h k l)$ and $(\bar{h} k \bar{l})$ are the same (neglecting effects such as possible differences in molecular charge density in the bulk and to-be-attached molecules at opposite faces). Although a difference in $E_{\text {att }}$ has been invoked to explain the polar morphology of urea obtained by sublimation (Docherty, Roberts, Saunders, Black \& Davey, 1993), its effect on morphology must be far less than that of solvent on crystal growth and morphology. Thus, in the absence of a difference in the effect of binding of solvent at the hemihedral faces $(h k l)$ and $(\bar{h} \bar{k} \bar{l})$, these two faces should grow at the same rate (Turner \& Lonsdale, 1950). A pronounced difference in growth rate must then be associated with differences in solvent-surface interactions. This principle was exploited in the family of plate-like crystals composed of amphiphilic molecules which pack in layers stacked head-to-tail in a polar arrangement (chain-like amphiphilic molecules normally pack head-to-head and tail-to-tail in the crystalline state). Thus, the polar plate-like crystals are hydrophobic at one plate face and hydrophilic at the opposite face. The idea was to ascertain the relative binding properties of polar solvents to the opposite hemihedral faces from the macroscopic wetting properties, in terms of contact angle measurements, and to correlate this effect with the relative rates of growth of the two crystal faces in the same solvents. In $\$ 4.1$ we had shown how the absolute configuration of the constituent molecules of chiralresolved alkylgluconamides, which pack in the desired polar arrangement (Fig. 7), may be assigned from the difference in wetting properties of the hydrophilic and 
hydrophobic faces. In $\mathrm{CH}_{3} \mathrm{OH}$ solution the polar platelike crystals of the octyl derivative $(n=8)$ were found to grow almost four times faster at the hydrophobic side than at the hydrophilic side (Wang, Leiserowitz \& Lahav, 1992). This result is completely in keeping with the observation that water and other polar solvents wet the hydrophilic face more strongly than the opposite hydrophobic face (see §4.1). It is noteworthy that at the hydrophilic face, solvent methanol can form $\mathrm{O}-\mathrm{H} \cdots \mathrm{O}$ hydrogen bonds with the terminal hydroxyl groups of two neighboring chains, since the translation separation distance between them is $c a 5 \AA$.

The theory that strong solvent-surface interactions inhibit crystal growth is also borne out by a comparison of the effect of tailor-made additives and acetic acid solvent on the morphology of the polar crystals of $N$-(cinnamoyl)alanine, already discussed in $\$ 4.1$. A benchmark study on the effect of solvent on growth of polar crystals was carried out by Wells (1949). He found that in aqueous solution, the $\alpha$-form of resorcinol $(1,3-$ dihydroxybenzene), which crystallizes in space group $P n a 2_{1}$, grows unidirectionally along the polar $c$-axis. The $\{011\}$ faces at one end of the polar axis have 'proton donor' properties and the $\{0 \overline{1} \overline{1}\}$ faces at the opposite end 'proton acceptor' properties. However, Wells could not fix the absolute direction of growth along the polar axis. This assignment was made with the use of tailor-made additives (Wireko et al., 1987). The unidirectional growth in aqueous solution occurs at the 'proton acceptor' $\{0 \overline{1} \overline{1}\}$ faces. This observation was explained in terms of stronger binding of water to the 'proton acceptor' $\{0 \overline{1} \overline{1}\}$ faces (Wireko et al., 1987; Davey, Milisavljevic \& Bourne, 1988). However, these analyses were not completely irrefutable, hinting that other growth mechanisms might also be responsible. Thus, we describe the next experiments on the polar crystals of $\gamma$-glycine and racemic alanine as well as urea, where different mechanisms were invoked to account for their growth behavior.

\subsection{A 'relay' mechanism of crystal growth as a result of selective solvent binding}

An entirely different approach involved strong selective adsorption of solvent at a subset of molecular surface sites, of say type $B$, and repulsion of solvent at the remaining set of surface sites, of say type $A$, on the crystal face. This is depicted in Scheme 7, where we emphasize the difference between the two types of sites by assuming a corrugated surface such that the $A$-type site is a cavity and the $B$-type site is on the outside upper surface of the cavity. Scheme 7(a) shows the $B$-type sites blocked by solvent $S$ and the $A$-type sites unsolvated. Thus, solute molecules can easily fit into $A$-type sites. However, once docked into position [Scheme 7(b)], the roles of the $A$ - and $B$-type sites are essentially reversed and the solvent molecules which originally were bound to $B$-type sites would be repelled since they now occupy $A$-type sites. This cyclic process can lead to fast growth by a kind of 'relay' mechanism.

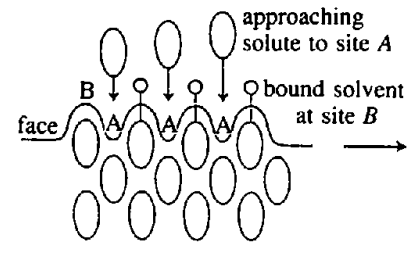

(a)

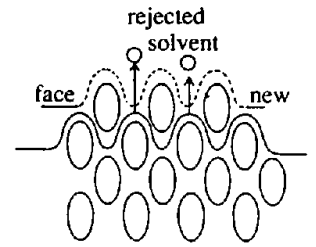

(b)
Scheme 7.

In such a situation, where desolvation is rate limiting, we have implicitly made use of the proposal that the free energy of incorporation of a solute molecule helps to displace bound solvent. This relay mechanism may be demonstrated by a crystal with a polar axis and the experiment involves a comparison of the relative rates of growth at the opposite poles for the different solvents, as exemplified by the growth and dissolution of the crystals of $(R, S)$-alanine and the $\gamma$-form of glycine in different solvents (Shimon, Vaida, Addadi, Lahav \& Leiserowitz, $1990,1991)$. These two crystals have similar packing features and so the discussion is confined to $(R, S)$ alanine. As already described in $\$ 4.1$, the zwitterionic molecules are aligned so as to expose $\mathrm{CO}_{2}^{-}$groups at one end of the polar $c$-axis and $\mathrm{NH}_{3}{ }^{+}$groups at the opposite end. The crystal has a polar morphology, with the $\mathrm{CO}_{2}^{-}$ groups emerging at the flat $-c$ end, as shown in Fig. 21. The $(00 \overline{1})$ face at the carboxylate end contains cavities. This face grows and dissolves faster in aqueous solution than the 'smoother' amino face [Fig. 22(a)]. We propose that the faster growth at the $(00 \overline{1})$ side of the crystal in aqueous solution is due to the relay mechanism of growth, according to the following arguments. The water molecules may be strongly bound by hydrogen bonds to the outermost layer (001) of $\mathrm{CO}_{2}^{-}$groups. In contrast, the
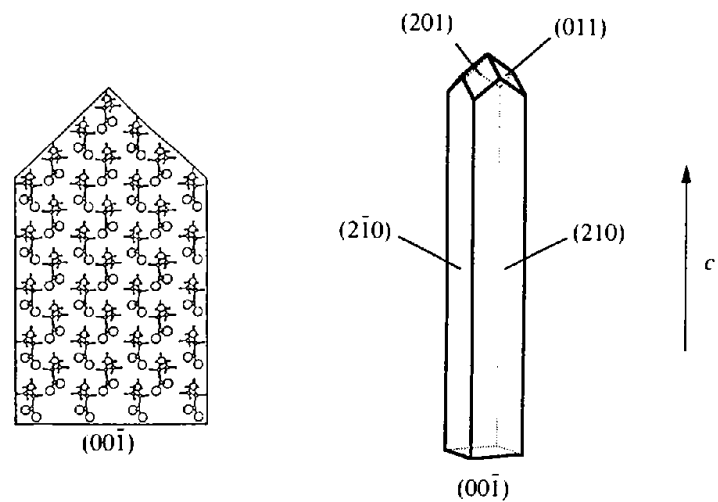

Fig. 21. Packing arrangement of $(R, S)$-alanine delineated by crystal faces, as viewed down the $b$-axis. The capped faces at the $+c$ end of the polar axis expose $\mathrm{NH}_{3}^{t}$ and $-\mathrm{CH}_{3}$ groups at their surfaces, the opposite $(00 \overline{1})$ face exposes carboxylate $\mathrm{CO}_{2}^{\overline{2}}$ groups. 
pockets act as proton acceptors for the $\mathrm{NH}_{3}^{+}$groups of the solute molecules. Replacement of the solute $\mathrm{NH}_{3}^{+}$moiety by solvent water within the pockets yields repulsive or, at best, weakly attractive interactions. The pockets will therefore be weakly hydrated and so relatively easily accessible to approaching solute molecules (Fig. 23). There is, however, an alternative mechanism which may account for the faster growth at the $-c$ end: a higher crystal attachment energy at the $(00 \overline{1})$ end of the crystal than at the opposite end. We could demonstrate that the relay mechanism is the more operative on the following basis: we predicted that methanol molecules could bind into the pockets at the $(00 \overline{1})$ face. The methyl group of this cosolvent molecule can form attractive, albeit weak, $\mathrm{C}-\mathrm{H}$...O interactions within the pocket and the $\mathrm{OH}$ group can form a hydrogen bond to the $\mathrm{CO}_{2}^{-}$group at the surface of the pocket. In keeping with prediction, crystals of $(R, S)$-alanine in $80 \%$ methanol:water mixture grow faster at the $+c$ amino end of the crystal than at the $-c$ carboxylate end [Fig. 22(b)], indicating that methanol blocks solute access to the pockets more effectively than water at the $(00 \overline{1})$ face.

This work implies that for those crystals which expose at each face set of different surface sites, the average binding energy of solvent to a face may not be a sufficient criterion for establishing the relative morphological importance of the different faces. It may be necessary to assess the ease of replacement of solvent by solute at each of the different surface sites, as well as the different modes of docking the solute molecules at the surface.

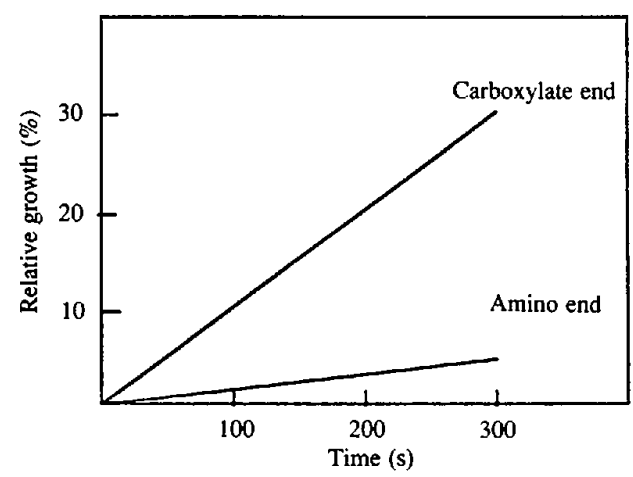

(a)

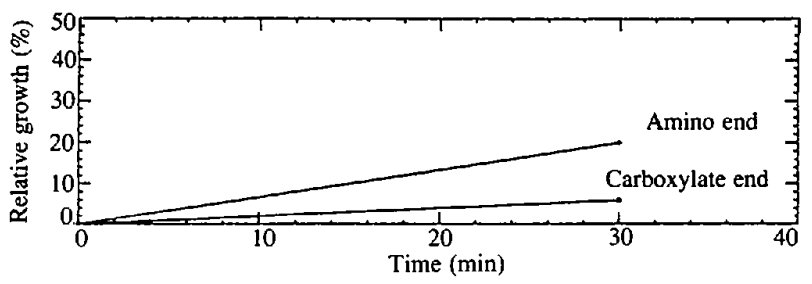

(b)

Fig. 22. Graph of the relative growth at opposite poles of the polar axis of $(R, S)$-alanine crystals: $(a)$ in water and $(b)$ in 4:1 methanol:water mixture.
For example, Boek, Feil, Briels \& Bennema (1991) calculated the theoretical growth form of tetragonal urea and found that it is almost cube-like and compares well with those of crystals from ethanol or benzene. However, the theoretical form is very different in shape from the [001] needles obtained from aqueous solution. Boek \& Briels (1992) performed molecular dynamics simulations of interfaces between water and crystalline urea. They argued that the observed needle morphology cannot be explained using a simple layer model because of strong adsorption of water to the relevant faces. By examining the interface between a saturated aqueous urea solution and crystalline urea, Boek (1993) explained the [001] needle morphology in terms of wrongly and randomly absorbed urea molecules to the $\{110\}$ side faces, so providing an increased interfacial entropy. It is noteworthy that oxamide crystals grown from aqueous solution contain ca $2 \%$ of wrongly oriented oxamide molecules in the lattice (Swaminathan \& Craven, 1982).

\section{Control of crystal phase formation}

Any crystallization process must cross the nucleation barrier. It is generally assumed that as the molecules start to associate in supersaturated solutions, they form embryos with structures resembling those of the to-begrown crystals. For polymorphic systems embryos of all the observed polymorphs are probably formed. Each type of embryo would structurally resemble the crystal into which it will eventually develop. If this hypothesis is correct, one may use the structural information of the mature crystals to design inhibitors of a particular crystalline phase. The overall consequence of this stereo-

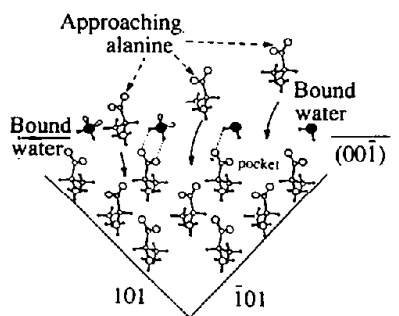

(a)

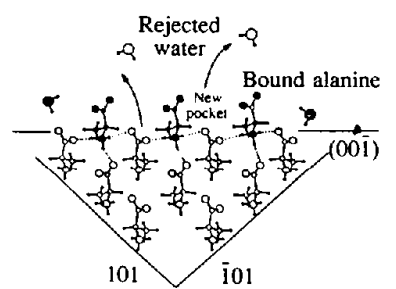

(b)
Fig. 23. Schematic representation of the (001) face of $R, S$-alanine during crystal growth. (a) In this view, approaching solute alanine molecules are depicted as about to be bound within the pockets of the $(00 \overline{1})$ face. Also shown are water molecules bound to the outermost $\mathrm{CO}_{2}^{-}$ groups of this face. The pockets remain poorly solvated because lone-pair-lone-pair oxygen-oxygen repulsions inhibit (similar to that described earlier in the asparagine-aspartic acid system, in \$5.4) the binding of water within them. (b) In this view, the newly adsorbed alanine molecules are each bound via three $\mathrm{NH} \cdots \mathrm{O}$ hydrogen bonds. The previously bound water molecules are shown being rejected by $\mathrm{O}$ (water) $\cdots O$ (carboxylate) lone pair-lone pair repulsions. Note the formation of new unsolved pockets. 
specific inhibition process can be that the unaffected phase, if less stable, will grow by kinetic control. We shall consider several systems where the logic of the above arguments apply. We first discuss the synthesis of chiral enantiomerically pure material from nonchiral reagents. This will be followed by a method for the kinetic resolution of enantiomers and the induced crystallization of a particular polymorph. Finally, the induced nucleation of a particular phase which acts as a template for the nucleation and further growth of the more stable phase into a macroscopic crystal will be discussed.

\subsection{Amplification of chirality by crystallization with chiral additives}

The solid state may be used for the synthesis of chiral enantiomerically pure material from nonchiral reagents by precipitation of nonchiral molecules in appropriately packed chiral crystals, followed by a lattice-controlled reaction (Green, Lahav \& Rabinovich, 1979; Addadi et al., 1980). However, crystallization from achiral solvents generally yields $\{R\}$ - and $\{S\}$-crystals in equal amounts. In order to obtain enantiomerically pure material, it is necessary to inhibit the nucleation of one of the two chiral crystal phases. This was achieved in the following way. Molecular dienes were 'engineered' to pack in chiral crystals (Addadi \& Lahav, 1979; Addadi, van Mil \& Lahav, 1982; van Mil, Addadi, Gati \& Lahav, 1981, 1982), in which the molecules are stacked by translation such that the neighboring C..C double bonds are in appropriate proximity for photodimerization (Scheme 8). UV irradiation of a single crystal yields dimers, trimers and oligomeric products of a single chirality $P_{r}$ or $P_{s}$, dependent upon the chirality of the starting crystal. Thus, the handedness of the molecular stacking arrangement is retained in the structure of the oligomer. A net asymmetric induction of one of the crystalline phases was achieved when crystals were grown in the

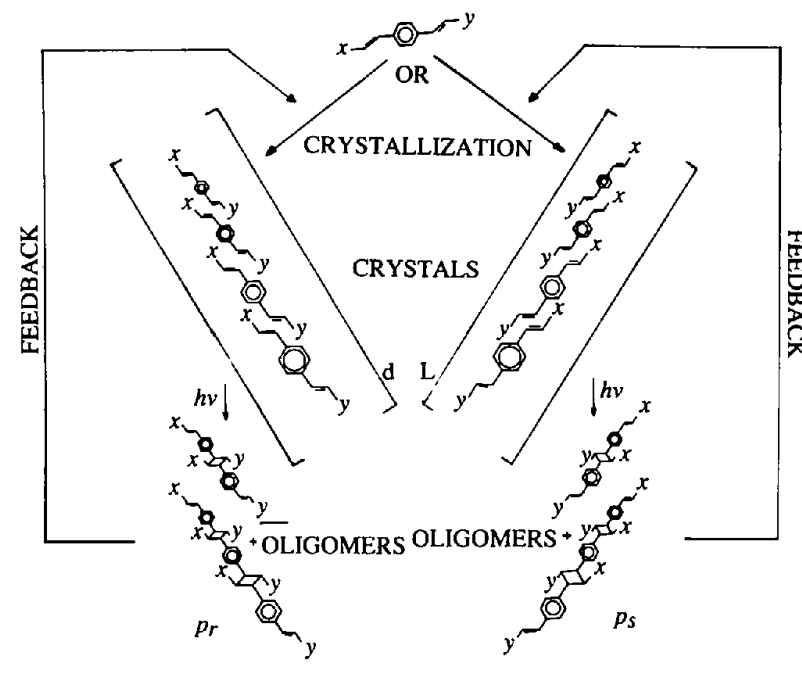

Scheme 8. presence of oligomers of a single chirality (i.e. $P_{r}$ or $P_{s}$ ) obtained from a large single crystal. In all experiments it was observed that a large excess was formed of the phase which was enantiomorphic to that in which the additive itself had been generated: namely, $R$-crystals were obtained from a solution containing $P_{s}$ additives, and vice versa.

A natural extension of this work led to the suggestion of a new method for kinetic resolution of a racemic mixture crystallizing in the form of a conglomerate of $\{R\}$ - and $\{S\}$-crystals (Addadi, van Mil \& Lahav, 1981; Addadi et al., 1982), as described below.

\subsection{Resolution of enantiomers}

Racemic mixtures of molecules which crystallize as a conglomerate yield two enantiomorphous equienergetic crystalline phases. Kinetic resolution of the conglomerate may be achieved with the assistance of tailormade chiral-resolved inhibitors which would, in solution, bind enantioselectively and stereoselectively to only one of the enantiomeric crystalline phases and so prevent its eventual growth. This principle has been demonstrated for many systems (Addadi et al., 1982; Addadi, Weinstein, Gati, Weissbuch \& Lahav, 1982; Zbaida et al., 1987). There are no particular requirements on molecular packing, although we may state that the higher the point symmetry of the crystal, the more different orientations in the lattice the constituent molecules may occupy, making the inhibition process more pronounced since the additive would be adsorbed on more faces. The kinetic resolution may encompass preferred precipitation from a racemic mixture in solution of one enantiomorph at the expense of the more stable racemic crystal phase. Naturally the energy gap between the racemic crystal and the mixture of resolved enantiomorphs must be relatively small. For example, racemic histidine. $\mathrm{HCl}$ precipitates, at $298 \mathrm{~K}$, as a stable racemic dihydrate crystal and a metastable conglomerate of resolved monohydrate crystals. Addition of as little as $1 \%$ of the chiral-resolved poly-( $p$-acrylamido- $S$ phenyalanine) additive induced resolution with quantitative yield of the desired enantiomer (Weissbuch, Zbaida, Addadi, Lahav \& Leiserowitz, 1987).

\subsection{Induced crystallization of a polar polymorph}

We now consider the preferred precipitation of a crystal containing a polar axis, at the expense of the dimorph which is centrosymmetric and thus nonpolar. The concept is that in crystals with a polar axis all molecules are aligned in the same direction vis-à-vis the polar axis. In centrosymmetric crystals neighboring molecules are arranged in an antiparallel manner. These two arrangements are depicted in Scheme 9. Thus, an appropriate tailor-made additive will inhibit growth of the centrosymmetric form at opposite ends of the crystal, 
growth inhibition

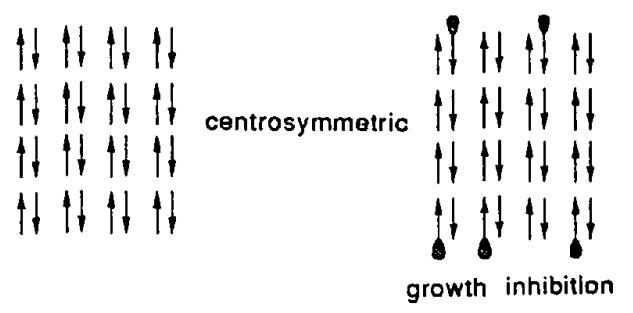

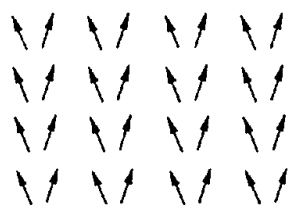

pure

(a) normal growth

grown in presence of additive

(b)

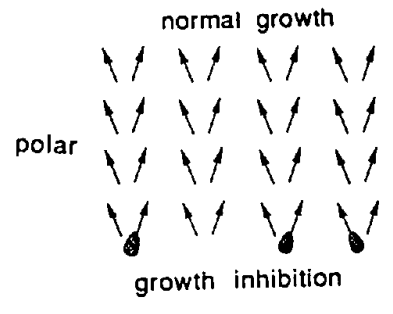

Scheme 9.

and so prevent its appearance, but will only inhibit growth of the polar form at one end of the crystal.

This concept has been demonstrated in two different systems. In $N$-(2-acetamido-4-nitrophenyl)pyrrolidine (PAN; 6a), the metastable polar form [Fig. 24(b)] displays optical second-harmonic generation. As little as $0.03 \%$ of the polymer $(6 b)$ inhibited precipitation of the pseudo-centrosymmetric stable form of PAN [Fig. (24a)], only allowing crystallization of the metastable form (Staab, Addadi, Leiserowitz \& Lahav, 1990).<smiles>CC(=O)Nc1cc([N+](=O)[O-])ccc1N1CCCC1</smiles>

(6a)<smiles>CCCOC(=O)NCCC(=O)Nc1cc([N+](=O)[O-])ccc1N1CCCC1</smiles>

(6b)

The other system involved preferential crystallization from aqueous solution of the $\gamma$-form of glycine, when grown in the presence of the additive racemic hexafluorovaline, $\left(\mathrm{CF}_{3}\right)_{2} \mathrm{CHCH}\left(\mathrm{NH}_{3}^{+}\right) \mathrm{CO}_{2}^{-}$(Weissbuch, Leiserowitz \& Lahav, 1994). As already mentioned, glycine usually crystallizes in the centrosymmetric $\alpha$ form from aqueous solutions, but litaka (1961) reported that glycine can precipitate in a polar $\gamma$-form from acetic acid or ammonia solutions. Perhaps the crystallization of $\alpha$-glycine in pure aqueous solution is promoted by the presence in solution of cyclic hydrogen-bonded dimers of glycine (see $\$ 7.1$ ), but whose formation is inhibited in acid or base solution, leading to the precipitation of the $\gamma$-form. The $\gamma$-form appears in space group $P 3_{1}$ (or $P 3_{2}$ ), arranged in a way similar to $(R, S)$-alanine crystal (Fig. 21 ), so that the molecules expose $\mathrm{CO}_{2}^{-}$groups at one end of the polar trigonal axis and $\mathrm{NH}_{3}^{+}$groups at the opposite end [Fig. 25(a)]. It was found that racemic hexafluorovaline is an effective inhibitor of growth of $\alpha$-glycine since it binds to the four $\{011\}$ faces and so blocks growth along both $b$ and the fast $c$-growing directions [Fig. 25(c)]. This behavior is in contrast to that of the common $\alpha$-amino acid additives which bind primarily to the $\{010\}$ faces and so retard the growth of $\alpha$-glycine only along the $b$-direction, inducing the formation of the $\{010\}$ plates, as described earlier in $\$ 5.1$. The abnormal behavior of hexafluorovaline may be understood in terms of its molecular structure and conformation, as found in its native crystal structure (Weissbuch, Frolow, Addadi, Lahav \& Leiserowitz, 1990); steric repulsion imposed by the hexafluoroisopropyl moiety prevents adsorption onto the $\{010\}$ face, but not at the $\{011\}$ faces to which it is strongly bound through charge-transfer interactions. Furthermore, hexafluorovaline does not inhibit nucleation and growth of the $\gamma$-glycine form because the additive is bound thereto primarily at the $\mathrm{NH}_{3}^{+}$end of its polar axis, the crystal growing unidirectionally along its polar $c$-axis at the $\mathrm{CO}_{2}^{-}$end. However, the hexafluorovaline molecules can be also adsorbed on the newly developed $\{\overline{1} 51\}$ side faces of the trigonal pyramidal crystals [Fig. 25(b)]. The salient lesson to be learnt from these experiments on glycine is that the inhibitor, to be effective, should bind along the fastgrowing directions. The antifreeze proteins in fish that inhabit the polar seas act in a similar way; they bind to the fast growing directions of the ice nuclei to inhibit

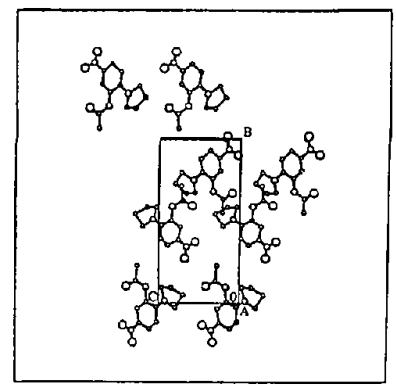

(a)

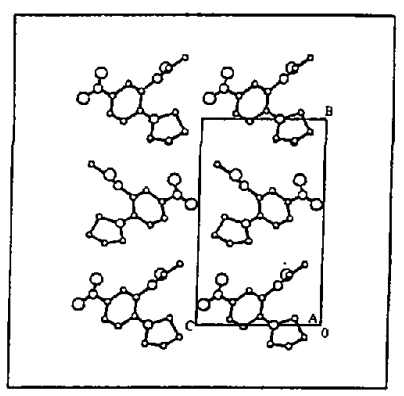

(b)
Fig. 24. Crystalline packing arrangements of PAN (6a): (a) stable form which is pseudocentrosymmetric and $(b)$ metastable form where the polar axis is along the vertical direction. 
growth (De Vries, 1984; Chakrabartty, Yang \& Hew, 1989; Knight, Chang \& De Vries, 1991).

\subsection{Information on early stages of nucleation via induced twinning of racemic alanine crystals}

Finally we examine a system in which a chiralresolved additive induces nucleation of a chiral phase from a racemic mixture in solution, but this step is followed by the eventual precipitation of the thermodynamically stable racemic crystal through an epitaxial structural fit between the two crystalline phases. The constituent molecules are alanine. The racemic $(R, S)$ crystal, already described in $\$ 4.1$ and $\$ 7$, crystallizes in the polar space group $P n a 2_{1}$. The molecules form parallel hydrogen-bonded chains along the polar $c$-axis, exposing $\mathrm{CO}_{2}^{-}$groups at one end of the polar axis and $\mathrm{NH}_{3}^{+}$groups at the opposite end (Fig. 21). The enantiomorphous crystals of $(R)$ - or $(S)$-alanine have very similar cell dimensions, but the hydrogen-bonded chains are arranged antiparallel in space group $P 2_{1} 2_{1} 2_{1}$, so exposing both $\mathrm{CO}_{2}^{-}$and $\mathrm{NH}_{3}^{+}$groups at each end of the $c$-axis. The energy gap between these two phases must be small and their solubilities are very similar. The racemic crystals exhibit a morphology (Fig. 21) of point symmetry $\mathrm{mm} 2$, in keeping with the space group. Precipitation of alanine from a racemic solution, when

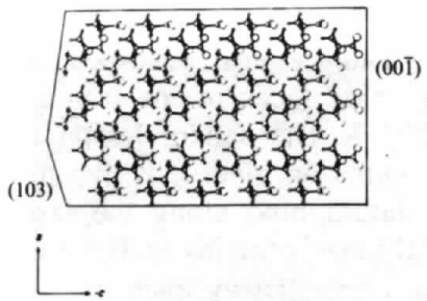

(a)

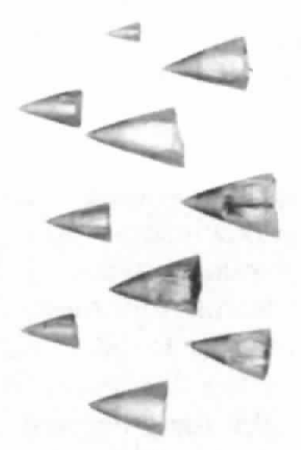

(b)
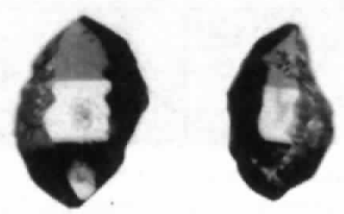

(c)

Fig. 25. (a) Packing arrangement of the $\gamma$-form of glycine, as delineated by the faces grown from aqueous solution containing acetic acid. (b) Photograph of $\gamma$-glycine crystals grown from aqueous solutions in the presence of hexafluorovaline additive (3\% wt). (c) Photograph of $\alpha$-glycine crystals grown from aqueous solution in the presence of a low concentration (1\% wt) of hexafluorovaline additive, showing inhibition of the four $\{011\}$ faces. grown in the presence of a chiral-resolved $\alpha$-amino acid additive, such as $(R)$-threonine, yields $(R, S)$-alanine crystals with an affected morphology, propeller-shaped and exhibiting 222 point symmetry [Fig. 26(a)]. Growth in the presence of the opposite enantiomeric additive $S$-threonine yields propellers of the mirror image (Weissbuch et al., 1994). The crystals expose $\mathrm{CO}_{2}^{-}$groups at each end of the propeller and so would appear to be twinned about the central $a b$-plane, in keeping with observation that the two crystal halves appear to be stitched across this central $a b$-plane. However, the two surfaces in contact across such a twinning plane would each expose $\mathrm{NH}_{3}^{+}$groups, which must be energetically unfavorable. One reasonable explanation for the observed effect is the inhibition of nucleation of both the racemic and the chiral form of alanine homomeric to that of the chiral-resolved additive in solution, leading to nucleation of the opposite enantiomer through the absence of steric interactions with the additive. Indeed this step was done by design. These chiral nuclei then act as templates at their two opposite $\{001\}$ faces for nucleation sites for epitaxial growth of $(R, S)$-alanine, yielding the 'twinned' crystals. The proposed structural arrangement at the epitaxial interface is shown in Fig. $26(b)$. This arrangement also brings about the point that

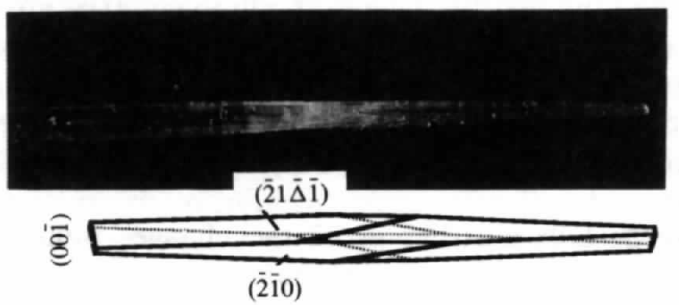

(a)

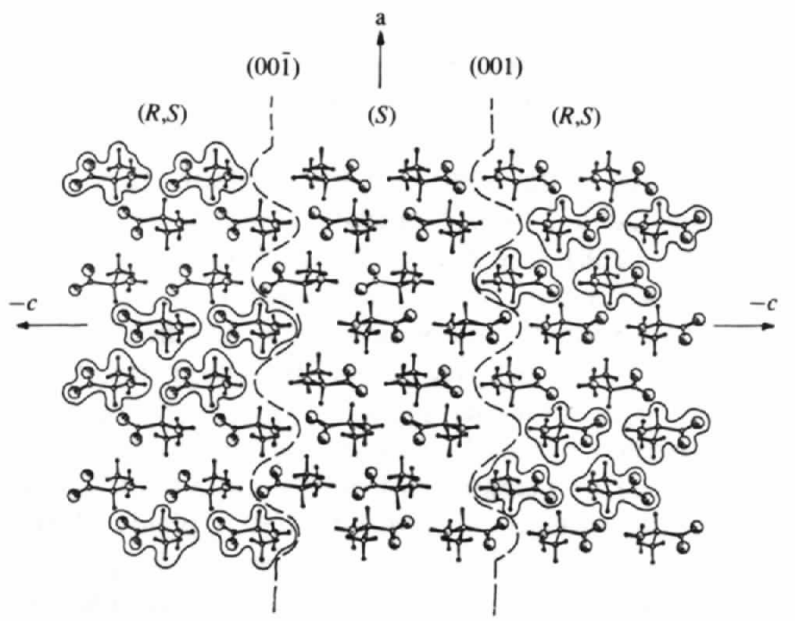

(b)

Fig. 26. (a) Twinned crystals of $(R, S)$-alanine grown in the presence of $1 \% R$-threonine additive. (b) Proposed structural arrangement showing the epitaxial growth of $(R, S)$-alanine at the $(001)$ and $(00 \overline{1})$ faces of a nucleus of $(S)$-alanine. 
each of the two $(R, S)$ to $(S)$ interfaces has half favorable $\mathrm{N}-\mathrm{H} \cdots \mathrm{O}$ hydrogen bonds and half unfavorable, but well-separated, $\mathrm{NH}_{3}^{+} \ldots{ }^{+} \mathrm{H}_{3} \mathrm{~N}$ electrostatic interactions, while twinning of the two $(R, S)$-domains without an interleaving $(S)$-layer would generate only unfavorable contacts. The tapered arms of the propeller are brought about by the enantioselective adsorption of the chiral additive at two opposite faces of the four $\{210\}$ side faces. The proposed mechanism of nucleation followed by epitaxial growth was substantiated by the observed growth of $(R, S)$-alanine from seeds of chiral-resolved alanine and by growth experiments involving various nonracemic $(R: S)$ mixtures. Furthermore, X-ray diffraction measurements of a twinned crystal revealed the following: a misalignment between the two halves, and reflections, but only from the crystal center, that are symmetry forbidden for space group $P n a 2_{1}$, and so could arise from a $P 22_{1} 2_{1} 2_{1}$ crystallite.

\section{9. 'Tailor-made' surfaces composed of amphiphilic monolayers for promotion of crystal nucleation}

Crystal nucleation is generally a heterogeneous process. This means that the activation barrier for nucleation is lowered by interaction with template surfaces. This process can happen at different levels of specificity, ranging from nonspecific adsorption to epitaxial growth. One can therefore envisage induced nucleation of desired crystalline structures, even with a specific crystal orientation, by designing appropriate nucleation promoters which match the structure of the crystal on a specific plane.

We described earlier in $\$ 3$ the changes induced in the $\alpha$-form of glycine crystals upon growth in the presence of various $\alpha$-amino acid additives. These changes involved morphology and enantiomeric segregation of the occluded additive molecules. Growth in the presence of hydrophobic $\alpha$-amino acid additives yielded glycine crystals at the air-solution interface, floating with their $\{010\}$ faces exposed to air, long before crystals appeared at the bottom of the crystallization vessel (Weissbuch et al., 1984; Weissbuch, Addadi, Leiserowitz \& Lahav, 1988). The $R$ - $\alpha$-amino acid additives such as leucine, norleucine, valine and $\alpha$-amino-octanoic acid induced the formation of pyramidal crystals floating with their $(010)$ faces exposed to air. By symmetry, the $S$ $\alpha$-amino acid additives induced the enantiomorphous (010)-oriented pyramids. Crystallization experiments in the presence of racemic hydrophobic additives yielded the solution surface covered with $\{010\}$ plates of both orientations. Crystallization experiments in the presence of optically pure hydrophobic $\alpha$-amino acid additives (e.g. $R$-leucine) and racemic hydrophilic $\alpha$-amino acids (e.g. racemic glutamic acid) yielded only (010)-oriented plates of glycine (Fig. 27). This orienting phenomenon was explained by the spontaneous aggregation of the hydrophobic $\alpha$-amino acid additives at the solution surface to yield nucleating domains. This proposed mechanism is also in keeping with surface tension measurements, according to which the hydrophobic $\alpha$-amino acids aggregate at the aqueous solution surface. Moreover, the chiral-resolved hydrophobic $\alpha$-amino acids, in their own crystal structures form hydrogen-bonded layers akin to that of $\alpha$-glycine (Benedetti, Pedone \& Sirigu, 1973; Weissbuch, Frolow, Addadi, Lahav \& Leiserowitz, 1990). The mechanism is also in keeping with HPLC measurements of the floating glycine crystals grown in the presence of the chiral-resolved hydrophobic $\alpha$ amino acid, which revealed very small amounts, if any, of the additive occluded in the crystals. Thus, it is conceivable that the hydrophobic $\alpha$-amino acid additives assemble at the solution surface into hydrogen-bonded layers, further stabilized by the binding of glycine solute molecules to form hydrogen-bonded bilayers, and which can induce the oriented nucleation of $\alpha$-glycine crystals at the solution surface.

These studies led directly to the use of water-insoluble $\alpha$-amino acid amphiphiles to induce oriented nucleation of $\alpha$-glycine crystals. Such a design of a synthetic template of a Langmuir monolayer of amphiphilic molecules spread at the air-solution interface and having their polar head groups in contact with the water proved to be a very expedient nucleating system. It has proven possible to induce, by virtue of a designed structural match between nucleator and nucleate, oriented crystallization of $\alpha$-glycine $\left({ }^{+} \mathrm{H}_{3} \mathrm{NCH}_{2} \mathrm{CO}_{2}^{-}\right)$under $\alpha$-amino acid monolayers such

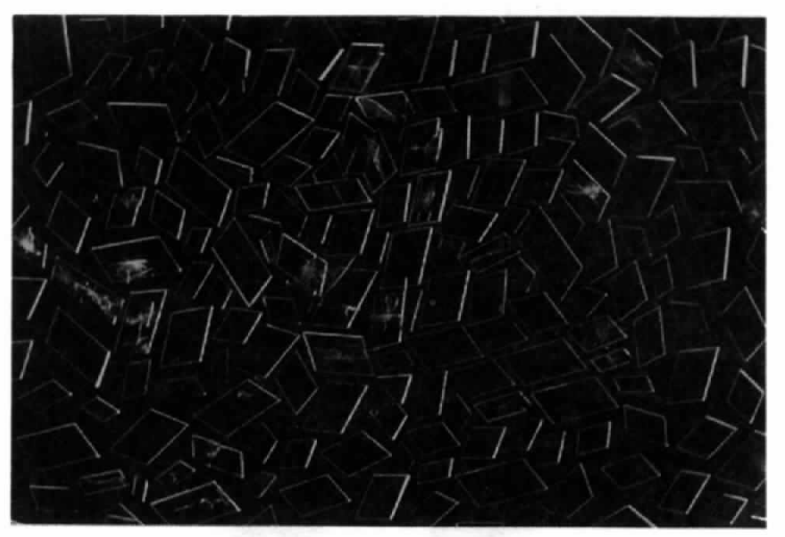

Fig. 27. A crust of oriented $\alpha$-glycine plate-like crystals floating on the surface, obtained from a solution containing $1 \% \mathrm{wt} / \mathrm{wt} R$-leucine and racemic glutamic acid. All the floating oriented plates expose their (010) faces to the air due to $R$-leucine, and glutamic acid is added to the solution to induce plate formation. This orientation is recognizable from the crystal shape, since for the majority of the crystals the long edge of each crystal is parallel to the $c$-axis and the short edge is parallel to $a$. For those crystals which appear to be reversed, of which there are approximately five or six in this photograph, the correct (010) orientation was confirmed by X-ray diffraction measurements. For such crystals the long edge was parallel to the $a$-axis and the short edge to $c$. 
as palmitoyl lysine, $\mathrm{C}_{15} \mathrm{H}_{31} \mathrm{CONHC}_{4} \mathrm{H}_{8} \mathrm{CHNH}_{3}^{+} \mathrm{CO}_{2}^{-}$ (Landau, Popovitz-Biro, Levanon, Leiserowitz \& Lahav, 1986; Landau et al., 1989). Several other types of crystals have since been nucleated at the water surface by Langmuir monolayers through either a (partial) structural fit or an electrostatic attraction. These include $p$ hydroxybenzoic acid (Weissbuch, Berkovic, Leiserowitz \& Lahav, 1990), silver propionate (Weissbuch, Majewski et al., 1993), sodium chloride (Landau, Popovitz-Biro, Levanon, Leiserowitz \& Lahav, 1986), nanocrystals of $\mathrm{PbS}$ (Zhao, Yang, McCormick \& Fendler, 1992) and CdS (Fendler, 1993), barium sulphate (Heywood \& Mann, 1992a,b) and the vaterite and calcite form of $\mathrm{CaCO}_{3}$ (Mann, 1988; Mann, Heywood, Rajam \& Birchall, 1988). The work on $\mathrm{CaCO}_{3}$ was done as part of model studies on biological mineralization.

We shall make brief mention of the crystalline selfaggregating properties and packing arrangements of the Langmuir monolayers, as established by grazing incidence X-ray diffraction (GID). This will be followed by an outline on the effect of solvent molecules on the growth, dissolution and packing structures of the amphiphilic monolayers. We shall also describe the design of mixed monolayers in order to glean a measure of the critical size of induced nuclei of the threedimensional crystals at the monolayer-solution interface. Finally, we examine the role played by monolayers of aliphatic alcohols for the induced nucleation of ice.

\subsection{Crystalline self-assembly of amphiphilic monolayers on the water surface}

Structural information on the induced nucleation of the crystals of $\alpha$-glycine, $p$-hydroxybenzoic acid, silver propionate and ice at the monolayer interface has been independently gleaned by determination of the twodimensional crystal structures of the monolayers on the liquid surface (Wolf et al., 1987; Jacquemain et al., 1990; Weissbuch, Majewski et al., 1993). This has been achieved by grazing incidence $\mathrm{X}$-ray diffraction (GID) using synchrotron radiation.

Evidence for the formation of self-organized twodimensional crystalline aggregates (crystallites) composed of water-insoluble amphiphilic molecules, at the air-water interface, has until recently been indirect; it has generally been assumed that the degree of lateral ordering in the uncompressed state is very low, if at all existent. The newly developed method of GID is a powerful tool for exploring this field (Als-Nielsen \& Kjaer, 1989; Barton et al., 1988; Als-Nielsen \& Mohwald, 1992; Jacquemain et al., 1992) since it allows for the detection of two-dimensional crystalites formed by the amphiphilic molecules and for the determination of their size and structure almost at the atomic level. Using GID, the two-dimensional crystallinity of amphiphilic monolayers in the relaxed state (i.e. where the monolayer coverage on the water surface is sufficiently low, e.g.
$<80 \%$, so that the monolayer surface pressure is very low) was shown to be a widespread phenomenon. This was demonstrated for a variety of amphiphiles, such as long fluorocarbon chain $\alpha$-amino acids (Jacquemain et al., 1990) and carboxylic acids (Dutta et al., 1987; Barton et al., 1988, 1992), hydrocarbon chain alcohols, amides, carboxylic acids (Jacquemain et al., 1991) and their cadmium or calcium salts (Böhm et al., 1994). Indeed the GID spectrum of the cadmium arachidate monolayer (Leveiller et al., 1991) gave direct evidence of an ordered counterionic cadmium layer bound to the carboxylate head group. This result of an ordered counterionic layer is in agreement with induced crystallization of inorganic salts at the Langmuir monolayer interface (Landau, Popovitz-Biro, Levanon, Leiserowitz \& Lahav, 1986; Landau et al., 1989; Zhao, Yang, McCormick \& Fendler, 1992; Mann, 1988; Mann, Heywood, Rajam \& Birchall, 1988). Analysis of the GID data using structure-factor calculations, sometimes complemented by lattice energy calculations yielded two-dimensional crystal structures almost at atomic resolution (Leveiller, Jacquemain, Leiserowitz, Kjaer \& Als-Nielson, 1992; Leveiller et al., 1994; Weissbuch, Leveiller et al., 1993; Wang et al., 1994; Böhm et al., 1994).

\subsection{Effect of solvent and tailor-made auxiliaries on growth, dissolution and structure of amphiphilic monolayers}

Amphiphilic monolayers on the water surface are an ideal vehicle for study of the effect of solvent on two-dimensional (and by extension, three-dimensional) crystal growth and dissolution. Two-dimensional crystals have an advantage over their three-dimensional counterparts, because solute molecules present in the aqueous subphase only come into contact with the polar head groups of the monolayer on a well characterized interface. Moreover, GID offers the possibility to monitor in real time, changes in two-dimensional crystallinity. We have been able, by GID, to demonstrate that solute can retard, or inhibit, the growth or induce dissolution of monolayer crystallites. For example (Jacquemain et al., 1990), the left part of Fig. 28(a) shows that 10 min after the monolayer of perfluorododecyl aspartate (PFA), $\mathrm{C}_{10} \mathrm{~F}_{21} \mathrm{C}_{2} \mathrm{H}_{4} \mathrm{OCOCH}_{2} \mathrm{CH}\left(\mathrm{NH}_{3}^{+}\right) \mathrm{CO}_{2}^{-}$, was deposited over an undersaturated solution of glycine, no diffraction peak was observed and it took around $30 \mathrm{~min}$ before crystalline domains could be detected. The inhibition can be understood from the top part of Fig. 28(b), which shows how one glycine molecule can bind to two neighboring molecules of the $\alpha$-amino acid monolayer with strong hydrogen bonds, thus hindering their assembly. The formation of this complex is in kinetic competition with that of the two-dimensional crystalline hydrogenbonded bilayer, which is composed of quasi-dimers of PFA and glycine [Fig. 28(b), top]. Once the PFA monolayer crystallizes, glycine solute molecules together with 
PFA form a pseudo-centrosymmetric hydrogen-bonded heterobilayer which provides additional stability to the system. Use of $\beta$-alanine, ${ }^{+} \mathrm{H}_{3} \mathrm{NC}_{2} \mathrm{H}_{4} \mathrm{CO}_{2}^{-}$, as a solute molecule instead of glycine had an entirely different effect on the two-dimensional crystals of uncompressed PFA; it did not strongly inhibit growth as dramatically as glycine, but caused a complete dissolution of the PFA crystals over $2 \mathrm{~h}$, as shown by GID measurements [Fig. 28(a), right]. This dissolution was rationalized in terms of a strong interaction of $\beta$-alanine with the amino acid head groups of neighboring surfactant molecules leading to a disruption of the two-dimensional crystals [Fig. $28(b)$, bottom], since $\beta$-alanine cannot form a hydrogenbonded heterobilayer with PFA as may glycine.

The second example involves the different hydrogenbonding properties of the cosolvents formamide $\left.(\mathrm{HCONH})_{2}\right)$ and formic acid $\left(\mathrm{HCO}_{2} \mathrm{H}\right)$ in aqueous solution, which have a drastic effect on the crystallinity and structure of films of aliphatic amides and carboxylic acids (Weinbach et al., 1993). Arachidamide, $\mathrm{C}_{19} \mathrm{H}_{39} \mathrm{CONH}_{2}$, on pure water forms a crystalline monolayer according to the Bragg peaks displayed by the GID pattern (Fig. 29). Analysis of the X-ray intensity profiles along the Bragg rods (not shown in Fig. 29) yielded a tilt of the molecular hydrocarbon chains of ca $18^{\circ}$ vis-à-vis the water surface. A $1 \mathrm{M}$ formamide solution as subphase, instead of pure water, did not have a detrimental effect on the monolayer crystallinity, according to the Bragg peak profiles (Fig. 29), but there was a change in structure since the molecular chains were vertically aligned on the subphase, as deduced from the Bragg rod intensity profiles. Replacing formamide by formic acid aqueous subphase did not change the Bragg peak pattern for a $0.3 \mathrm{M}$ solution (Fig. 29), but $1 \mathrm{M}$ formic acid solution inhibited the formation of two- dimensional crystalline $\mathrm{C}_{19} \mathrm{H}_{39} \mathrm{CONH}_{2}$ monolayers (Fig. 29). The essential difference in binding of formamide and formic acid to the amide groups of the amphiphile is depicted in Scheme 10. It involves the replacement of a $-29 \mathrm{kj} \mathrm{mol}^{-1}$ attraction between monolayer-bound formamide molecules by a $8 \mathrm{kj} \mathrm{mol}^{-1}$ repulsion between lone-pair electrons of monolayer-bound formic acid molecules. We may deduce that the lone pair-lone pair repulsion between bound formic acid molecules inhibits two-dimensional crystal growth of the $\mathrm{C}_{19} \mathrm{H}_{39} \mathrm{CONH}_{2}$ monolayer. We note that this inhibition is essentially an extension of the effect of tailor-made carboxylic acid additives on the morphology of primary amide crystals discussed in $\$ 2$ and $\$ 5.4$.

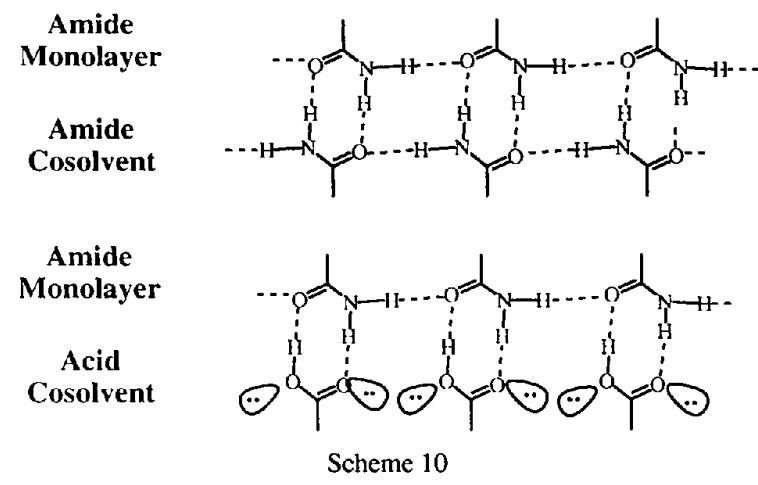

An increase in concentration of the formamide solution to $7 \mathrm{M}$ revealed by GID [Fig. 30(a)] the formation of two coexisting crystalline phases of arachidamide, a trilayer and a monolayer (Weinbach et al., 1994). In the monolayer phase [Fig. 31(a)], the molecular hydrocarbon chains were aligned vertically with respect to the liquid surface. In the trilayer phase [Fig. 31(a)], the chains

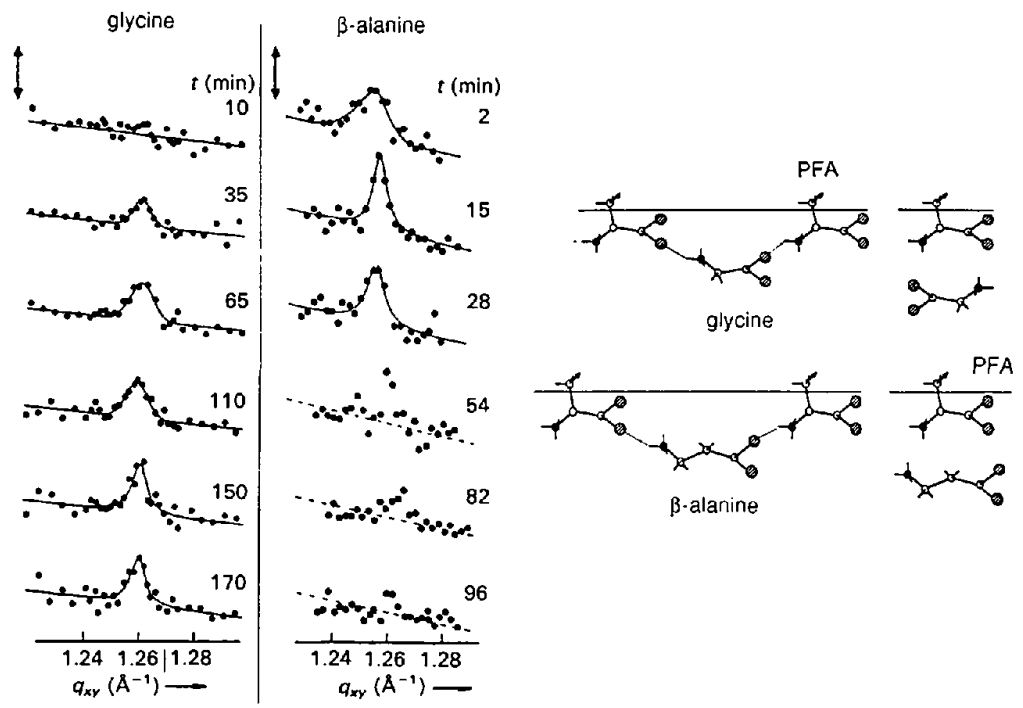

(a) (b)
Fig. 28. Uncompressed monolayer of PFA spread at an average molecular area of $45 \AA^{2}$ over various solutions. (a) GID measurements as a function of time after spreading: over glycine solution $(0.015 \mathrm{M}$; left); over $\beta$ alanine solution $(0.005 \mathrm{M}$; right). Note the increase in two-dimensional crystallinity in the former, and the decrease in the latter as indicated by the different time evolution of the peak. $q_{x y}(=4 \pi \sin \theta / \lambda)$ is the horizontal scattering vector parallel to the water surface. (b) Schematic drawings of 'bridging complexes' between PFA monolayer molecules and the solute molecules for glycine (top) and $\beta$-alanine (bottom). A potential quasidimer is illustrated for glycine, while the mismatch is depicted for $\beta$-alanine. Key: large stippled circles, $\mathrm{O}$; small empty circles, $\mathrm{C}$; full small circles, $\mathrm{N}$ atoms; $\cdots$, hydrogen bonds; $\rightarrow$, hydrophobic chains. 
were tilted $c a 30^{\circ}$ from the normal to the liquid surface. The pure formamide subphase yielded two phases in the trilayer form, the minor one tilted as before and the major phase with the molecular chains vertically

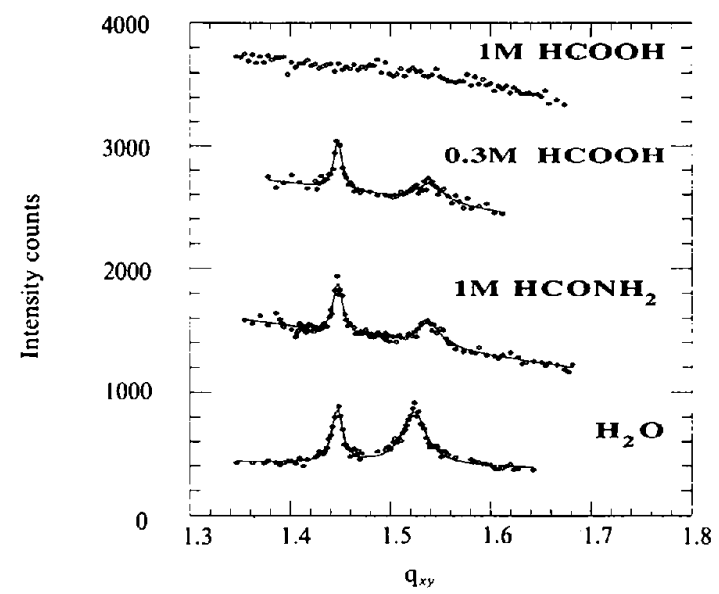

Fig. 29. GID diffraction pattern, showing the Bragg peaks of a monolayer of uncompressed arachidamide spread on various subphases. The horizontal axis $q_{x y}=4 \pi \sin \theta / \lambda$, where $\theta$ is the component of the Bragg angle parallel to the monolayer plane.

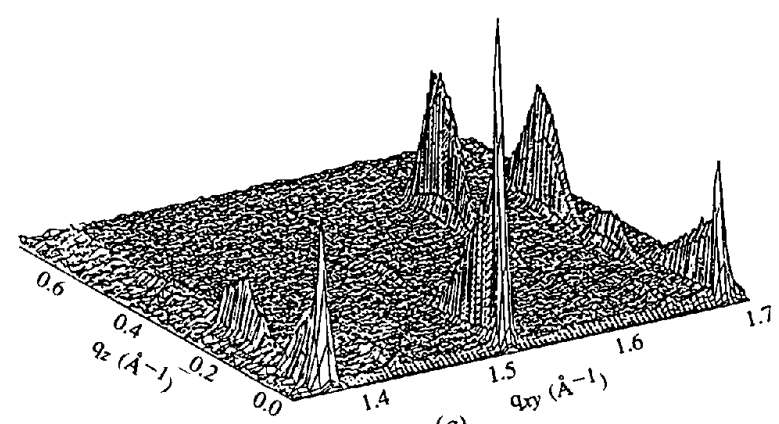

(a)

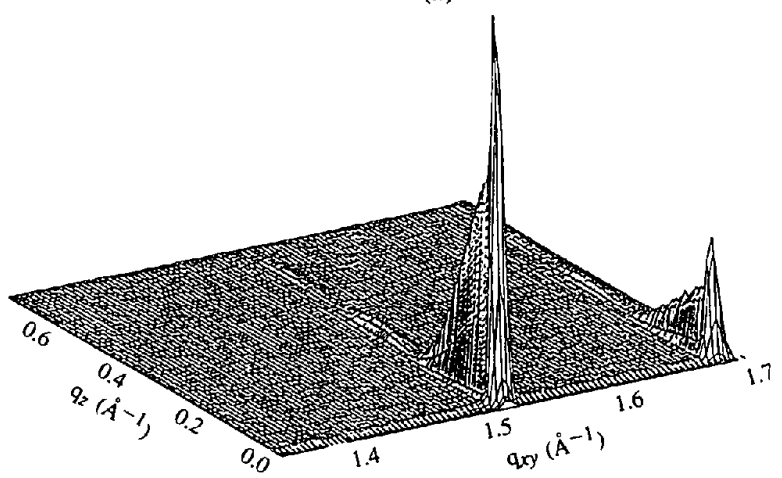

(b)

Fig. 30. Two-dimensional intensity plots $I\left(q_{x y}, q_{z}\right)$ of the GID pattern of arachidamide on $70 \%$ formamide-water subphase at $278 \mathrm{~K}$. The $q_{x y}$-axis describes the horizontal X-ray scattering vector $4 \pi \sin \theta / \lambda$, where $\theta$ is the component of the Bragg angle parallel to the water surface. The $q_{z}$-axis describes the vertical X-ray scattering vector. (a) Pure arachidamide and $(b)$ arachidamide containing $5 \% \mathrm{~N}$-methyl arachidamide. aligned. According to the GID pattern [Fig. 30(b)], it proved possible to inhibit the trilayer formation, yielding only the monolayer of the phase in which the molecules are vertically aligned [Fig. 31(b)], by deposition of the amphiphile together with the tailor-made additive $N$-methylarachidamide. This inhibition process is similar to the behavior of the tailor-made additives on growth of three-dimensional molecular crystals. Spontaneous formation of multilayers of amphiphiles on liquid surfaces may prove not to be a rare phenomenon. It has been found to occur for other amphiphilic systems such as $\mathrm{C}_{19} \mathrm{H}_{39} \mathrm{CONHCH}_{2} \mathrm{CH}_{2} \mathrm{CONH}_{2}$ on water (Weinbach et al., 1993). Indeed, the bolaamphiphiles $\mathrm{HO}-\left(\mathrm{CH}_{2}\right)_{n}-\mathrm{OH}(n=22,23,30)$ spontaneously form crystalline multilayers on the water surface (PopovitzBiro et al., 1994a,b; Majewski, Edgar et al., 1995).

\subsection{Mixed monolayers as a probe for mimicking the solid-liquid interface}

Although many experiments have been performed in which the crystal morphology can be understood in terms of strong binding of either solvent or additive molecules to specific faces, little work has been reported on the direct determination of the local structure at liquid-crystal interfaces. It is perhaps a consequence of the fact that the structure and dynamics at the solid-liquid interface is not easily amenable to direct study. Nevertheless, in situ synchrotron X-ray scattering studies have been recently carried out at the gold-electrolyte (Ocko, Wang, Davenport \& Isaacs, 1990; Wang, Davenport, Isaacs \& Ocko, 1992) and calcite-water (Chiarello, Wogelins \& Sturchio, 1993) interfaces, and the growth and dissolution of ammonium

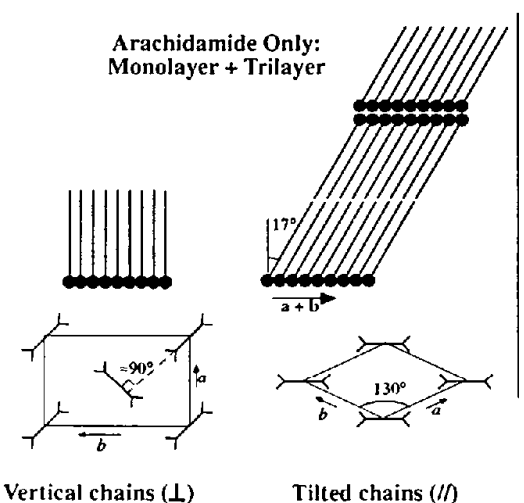

(a)
Arachidamide + Additive: Monolayer

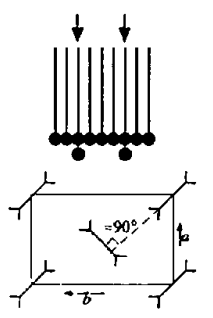

Vertical chains (1)

(b)
Fig. 31. Schematic representation of the packing arrangements of arachidamide on $70 \%$ formamide-water solution, showing side views (top) and views along the hydrocarbon chain axes (bottom). (a) Pure arachidamide. Two phases, one a monolayer with chain axes vertically aligned in a herringbone structure and the other a trilayer phase with the chains tilted and parallel. (b) Arachidamide plus additive $N$-methyl arachidamide. Monolayer phase with chains vertically aligned in a herringbone arrangement. 
dihydrogen phosphate, $\mathrm{NH}_{4} \mathrm{H}_{2} \mathrm{PO}_{4}$, in aqueous solutions (Cunningham, Gerson, Roberts, Sherwood \& Wojciechowski, 1991). A monolayer may be designed so as to partially mimic the solid-liquid structure and so be amenable to study by GID and X-ray reflectivity. Thus, we made use of a 1:1 mixed monolayer of longchain carboxylic acids and amines to mimic the structure of that crystalline face of $\beta$-alanine which exposes the highest concentration of $\mathrm{CO}_{2}^{-}$and $\mathrm{NH}_{3}^{+}$groups and which was found to be strongly inhibited on growth in $\mathrm{CdCl}_{2}$ aqueous solution (Gidalevitch, Weissbuch, Kjaer, Als-Nielsen \& Leiserowitz, 1994). We had found, both by GID and X-ray reflectivity measurements, an ordered binding of $\mathrm{Cd}^{2+}$ and $\mathrm{Cl}^{-}$ions to the head groups of the mixed monolayer. Moreover, addition of $\beta$-alanine to the $\mathrm{CdCl}_{2}$ subphase solution resulted in competitive binding of $\beta$-alanine molecules to the head groups of the monolayer.

\subsection{Mixed monolayers for the design of structured surfaces to induce three-dimensional crystallization}

The transfer of structural information from the monolayer to the three-dimensional crystal can provide evidence for a correlation between the size and structure of the monolayer as two-dimensional crystalline 'nuclei' and the tendency for formation of the three-dimensional crystals. The size, or lateral area, of the monolayer inducing nucleation of the three-dimensional crystals may be controlled using mixed monolayer films of type $A+B$, where the pure amphiphile $A$ is an efficient nucleator, whereas pure $B$ amphiphile is not. The oriented growth of three-dimensional crystals may be studied as a function of the molar ratio of the two components in order to establish the threshhold concentration required for efficient nucleation of the crystals (Weissbuch, Majewski et al., 1993).

One approach for the design of mixed monolayers with segregated domains of type $A$ and $B$ involves the use of molecules with a common moiety, structured so as to promote the formation of a homogeneous array of molecular moieties, common to $A$ and $B$. Molecule $A$, however, contains additional groups appropriate for the induced three-dimensional crystallization and which would also favor their segregation into domains. As $B$ we used $\mathrm{C}_{n} \mathrm{H}_{2 n+1} \mathrm{CONH}_{2}, n=19$, and as $A$ $\mathrm{C}_{n} \mathrm{H}_{2 n+1} \mathrm{CONHCH}_{2} \mathrm{CH}_{2} \mathrm{COOH}, n=19$. The hydrogenbonding amide group provides the driving force for a continous network of hydrocarbon chains. The head group of $A,-\mathrm{CH}_{2} \mathrm{CH}_{2} \mathrm{COOH}$, was chosen and indeed found to favor segregation, as shown in Scheme 11, and so induce the crystallization of a variety of layer compounds, for example, metal salts of carboxylic acids. The structural requirement for nucleation is that the crystal be composed of bilayers with the metal ions concentrated at the center of each bilayer. We chose silver propionate, $\mathrm{CH}_{3} \mathrm{CH}_{2} \mathrm{COOAg}$, as an appropriate compound. It was

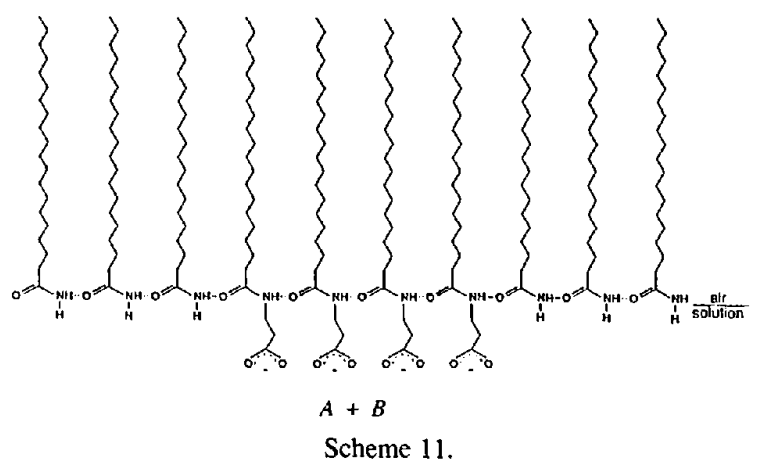

found that mixed monolayers $A$ and $B$ with a molar ratio $A: B$ down to $1: 10$ induced oriented formation at the airsolution interface of plate-like silver propionate crystals. Only few crystals, and with a different morphology, were obtained for a lower $A: B$ ratio or pure $B$. A randomly distributed monolayer mixture of two amphiphiles $A^{\prime}+\mathrm{B}^{\prime}$ did not induce crystallization of silver propionate, as did, for example, stearic acid $A^{\prime}$ and stearyl alcohol $B^{\prime}$ in the ratio as high as $1: 1$. The segregated nature of the $A+B$ domains was demonstrated independently by specular Xray reflectivity measurements. The presence of cadmium ions bound to neighboring $\mathrm{CO}_{2}^{-}$head groups was used as the probe to show the extensive juxtaposition of $A$ type molecules. The homogeneous and ordered nature of the $A+B$ mixed monolayers was demonstrated by GID for a variety of $A: B$ mixtures (over $\mathrm{CdCl}_{2}$ solution and pure water subphases). The remaining question to be addressed in this analysis is the size of the embedded $A$-type domains, which will provide information as to the size of the 'nuclei' of silver propionate en route to three-dimensional crystallization. Such information represents one of the principal unknowns in crystallization processes.

\subsection{Induced nucleation of ice by monolayers of long- chain alcohols}

Pure water can be supercooled to temperatures as low as $233-253 \mathrm{~K}$. Therefore, the inhibition or induction of the freezing point of ice, in particular through the role of auxiliaries, has far-reaching ramifications for the living and the non-living world. An intriguing example of inhibition involves the activity of antifreeze proteins in the blood serum of fish in polar seas, these proteins lower the freezing point by no more than $2 \mathrm{~K}$, but this small difference is vital for their survival (De Vries, 1984; Feeney, Burcham \& Yeh, 1986; Yang, Sax, Chakrabartty \& Hew, 1988). Promotion of ice nucleation, has been exploited in the induced precipitation of rain by silver iodide seeded in clouds (Vonnegut, 1947; Corrin \& Nelsen, 1968). On the other hand, it can result in wide-scale damage to nonconiferous plants in temperate climates by frost bacteria (Schnell \& Vali, 1972; Maki, Galyan, Chang Chien \& Caldwell, 1974; Lindow, Arny 
\& Upper, 1978; Lindow, 1983), which nucleate ice at temperatures as high as $271 \mathrm{~K}$.

Ice nucleation has been widely studied, yet the role played at the atomic level by auxiliaries involved in the promotion of ice nucleation is still unclear. Several mechanisms have been proposed such as a lattice or structural match between the substrate and the nucleated ice crystal (Fletcher, 1970; Warren, Corotto \& Wolber, 1986; Mizuno, 1989; Kajava \& Lindow, 1993), or an electric field present in the substrate which orients the water molecules to induce ice nucleation (Prappacher, 1963). To help unravel the complexity of this process, we designed experiments where such mechanisms can be studied in a systematic manner. There is a close expitaxial fit between the $a b$-cell axes of hexagonal ice $\left(a=b=4.5 \AA, \gamma=120^{\circ}\right)$ and of the two-dimensional cell of a compressed monolayer of the aliphatic long chain alcohol $\mathrm{C}_{21} \mathrm{H}_{43} \mathrm{OH}\left(a=b=4.5 \AA, \gamma=113^{\circ}\right)$ at just above $273 \mathrm{~K}$, as determined from GID measurements (Barton et al., 1988). We found that uncompressed monolayers of aliphatic long chain alcohols were very efficient ice nucleators (Gavish, Popovitz-Biro, Lahav \& Leiserowitz, 1990; Popovitz-Biro, Wang, Majewski, Leiserowitz \& Lahav, 1994). The induced freezing point of ice for $\mathrm{C}_{n} \mathrm{H}_{2 n+1} \mathrm{OH}$ with $n$ odd reached an asymptotic temperature just below $273 \mathrm{~K}$ for an upper value of $n=$ 31 , the freezing point for $n$ even reached a plateau of $265 \mathrm{~K}$ for $n$ in the upper range 22-30. The long-chain carboxylic acids $\mathrm{C}_{n} \mathrm{H}_{2 n+1} \mathrm{CO}_{2} \mathrm{H}$ are, by comparison, poor ice nucleators (Fig. 32).

GID studies of uncompressed monolayers of $\mathrm{C}_{n} \mathrm{H}_{2 n+1} \mathrm{OH}, n=13-31$, on water at $278 \mathrm{~K}$ revealed two-dimensional crystalline self-assembly and yielded

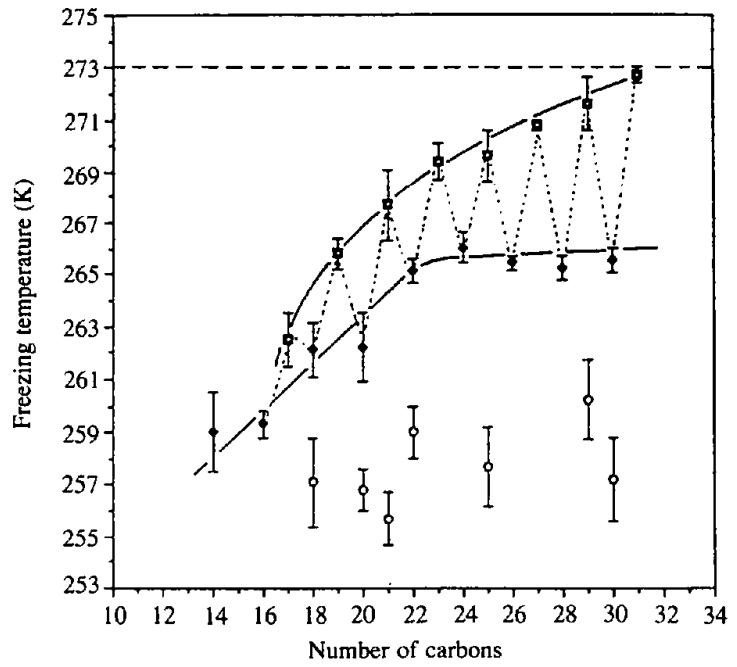

Fig. 32. Freezing points of drops of supercooled water covered by monolayers of alcohol, $\mathrm{C}_{n} \mathrm{H}_{2 n+1}+\mathrm{OH}$ ( $n$ even, filled diamond; $n$ odd, empty square), and carboxylic acid, $\mathrm{C}_{n} \mathrm{H}_{2 n+1}+\mathrm{CO}_{2} \mathrm{H}$ (empty circle). Freezing point curves are drawn separately for alcohols with $n$ odd and $n$ even. their packing arrangements to near atomic resolution and information on molecular thermal motion (Wang et al., 1994; Popovitz-Biro, Wang, Majewski, Leiserowitz \& Lahav, 1994; Majewski, Popovitz-Biro et al., 1995). The extent of two-dimensional crystalline order (i.e. coherence length) and the amount of crystalline material was less for monolayers, with $n=16$ and 20 , than for $n=23,30,31$ according to a GID analysis, the latter property being independently confirmed by FT-IR. The best lattice match to ice was for $n=30$ and 31 , the lattice match to ice being poorer for $n=13-20$ in terms of area per molecule, so correlating with their less efficient ice nucleation behavior. The packing arrangements of the hydrocarbon chains of monolayers $\mathrm{C}_{30} \mathrm{H}_{61} \mathrm{OH}$ and $\mathrm{C}_{31} \mathrm{H}_{63} \mathrm{OH}$ are very similar, but for the orientation of their $-\mathrm{CH}_{2} \mathrm{OH}$ moieties which are different, resulting in different ice nucleation behavior. Fig. 33 shows the packing arrangement of the monolayer of $\mathrm{C}_{31} \mathrm{H}_{63} \mathrm{OH}$. Further evidence for the role played by the orientation of the $\mathrm{C}-\mathrm{OH}$ group is the ice nucleating properties of alcohols $\mathrm{CH}_{3}\left(\mathrm{CH}_{2}\right)_{n} \mathrm{COX}\left(\mathrm{CH}_{2}\right)_{m} \mathrm{OH}(X=\mathrm{O}, \mathrm{NH})$. In these systems the freezing point was found to depend on the odd and even value of $m$, but not on that of $n$. The two-dimensional crystal structures of two ester-aicohols,

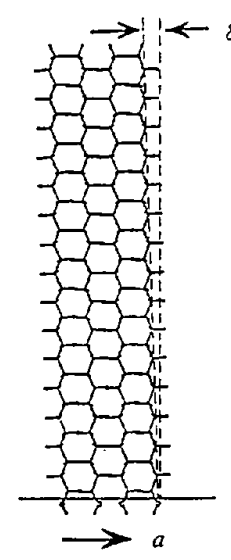

(a)

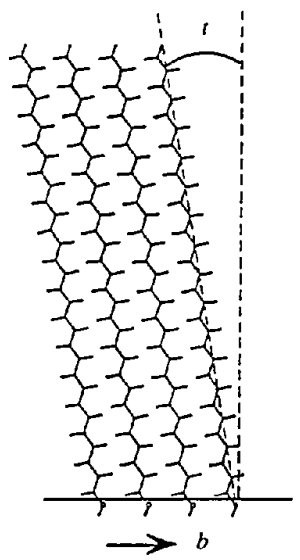

(b)

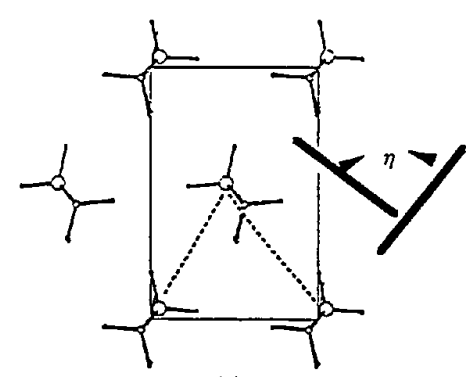

(c)

Fig. 33. Two-dimensional crystal structure of the $\mathrm{C}_{31} \mathrm{H}_{63} \mathrm{OH}$ monolayer on water at $278 \mathrm{~K}$, in which the two molecules in the unit cell are essentially related by glide symmetry along the $b$-axis. $(a),(b)$ and $(c)$ are views along the $a$-axis, the $b$-axis and down the molecular axis, respectively. 
$n=19, m=9,10$, on water were determined by GID and lattice energy calculations yielding distinctly different $-\mathrm{CH}_{2} \mathrm{OH}$ orientations. The effect of molecular motion of the alcohol chains on induced ice nucleation was also evident from the GID studies. The atomic displacement parameter (ADP) of $\mathrm{C}_{n} \mathrm{H}_{2 n+1} \mathrm{OH}$ increases with chain shortening from $n=31$ to 13 , which is compatible with the lowering in ice nucleation efficiency. For $n=31$, the value of ADP parallel to the plane of the monolayer is $c a$ $0.12 \AA^{2}$, for $n=19$ a value of $0.25 \AA^{2}$, and for $n=13$ the chain is almost a free rotator according to its large crosssectional molecular area (Majewski et al., 1995). The epitaxial nature of the induced ice nucleation is obvious from the similarity between the arrangement of $\mathrm{O}$ atoms in the (001) layer of ice and in the alcohol monolayer $\mathrm{C}_{n} \mathrm{H}_{2 n+1} \mathrm{OH}(n=30,31)$ over water, at a temperature of $278 \mathrm{~K}$, as depicted in Figs. 34(a) and (b). This epitaxial relation was confirmed by the electron diffraction pattern [Figs. 34(c) and $(d)$ ] from an essentially single crystallite of the $\mathrm{C}_{31} \mathrm{H}_{63} \mathrm{OH}$ monolayer on a single crystal of ice, in proper registry (Majewski et al., 1993). Recently we have grown under a $\mathrm{C}_{31} \mathrm{H}_{63} \mathrm{OH}$ monolayer, at a temperature just below $273 \mathrm{~K}$, (001) plates of ice crystals smooth enough that it was possible to detect the presence of the ordered monolayer on ice by GID (Majewski, Popvitz-Biro et al., 1994). Many of these ice crystals had a lateral coherence length as low as $25 \AA$, probably

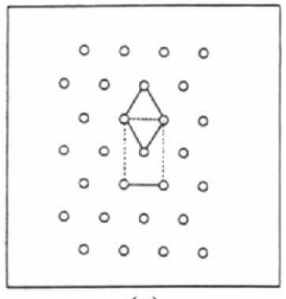

(a)

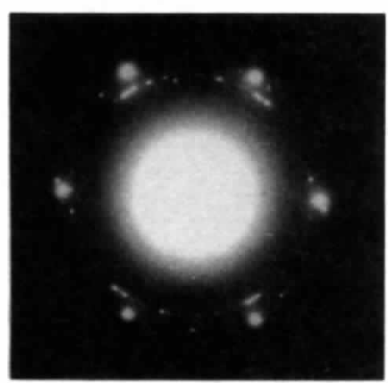

(c)

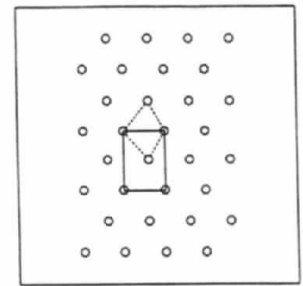

(b)

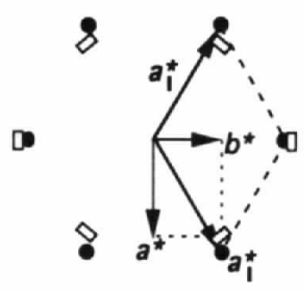

(d)
Fig. 34. Layer arrangements of the $\mathrm{O}$ atoms in $(a)$ an (001) layer of hexagonal ice and $(b)$ the alcohol $\left(\mathrm{C}_{n} \mathrm{H}_{2 n+1}+\mathrm{OH}, n=30,31\right)$ monolayers. The ice lattice is depicted in terms of the regular hexagonal cell (solid line) and a centered rectangular cell (dotted line). The regular rectangular lattice of the alcohol monolayers is drawn as well as a distorted hexagonal net jointing the $\mathrm{O}$ atoms. (c) Electron diffraction pattern from an essentially single crystallite of the $\mathrm{C}_{31} \mathrm{H}_{63} \mathrm{OH}$ monolayer on a single crystal of hexagonal ice. (d) Schematic relation between the reciprocal lattice of ice (filled circles) and of the monolayer (empty rectangles). by virtue of multiple ice nucleation sites. This value of $25 \AA$ provides rather direct information of an estimate on the upper limit of the critical size of ice nuclei as induced by the monolayer just below $273 \mathrm{~K}$, suggesting a cluster of about 100 water molecules.

\section{Induced nucleation of ice on surfaces of $\alpha$-amino acid crystals and by frost bacteria}

There are reports in the literature on induced ice nucleation that indicate mechanisms other than an epitaxial fit. For example, the onset temperature of ice nucleation by crystalline powders of chiral-resolved hydrophobic $\alpha$-amino acids such as valine, leucine and isoleucine was higher than the temperature induced by their corresponding racemic counterparts (Power \& Power, 1962; Barthakur \& Maybank, 1963; Parungo \& Lodge, 1967). These results were surprising because the chiral and racemic crystal forms of these amino acids resemble each other in molecular packing and crystal morphology. A comparative study of ice nucleation on isostructural faces of single crystals of chiral and racemic $\alpha$-amino acids showed that crystal polarity plays an important role (Gavish, Wang, Eisenstein, Lahav \& Leiserowitz, 1992), as described below.

The hydrophobic $\alpha$-amino acids pack in hydrogenbonded layers. In the chiral crystals, molecules within the layer are related by translation and interlinked by $\mathrm{N}-\mathrm{H} \cdots \mathrm{O}$ bonds, as in the $\alpha$-form of glycine [Fig. $3(a)$ ]. In the corresponding racemates, the molecules appear in a similar layer motif. The neighboring layers are interlinked by $\mathrm{N}-\mathrm{H} \cdots \mathrm{O}$ bonds to form bilayers. These bilayers are generated in the chiral crystals by (pseudo-) twofold symmetry and in the racemates by centers of inversion, as in the $\alpha$-form of glycine [Fig. $3(a)$. The chiral crystals are polar as a consequence of the monoclinic (pseudo-) twofold axis parallel to the plane of the bilayer. The racemic crystals are centrosymetric and hence are nonpolar. The platelike faces of the hydrophobic $\alpha$-amino acid crystals expose hydrocarbon residues according to water contact angles. Water vapor condensed on these faces at $273 \mathrm{~K}$ froze, on cooling, at temperatures higher by $3-5 \mathrm{~K}$ on the polar chiral amino acid crystals than on the corresponding centrosymmetric racemates. In a different type of experiment, the substrate crystals were cooled to $258 \mathrm{~K}$ before water vapor deposition. Ice crystals hexagonal in shape, and with the $c$-axis tending to be parallel to the face of the substrate, were found to emerge from cracks at their surfaces [Fig. 35(a)].

The above results were explained as follows: The structure of the plate faces of the polar and nonpolar $\alpha$-amino acid crystals are depicted schematically in Figs. 35(c) and (d). Both faces contain cracks that expose the hydrophobic as well as the hydrophilic $\mathrm{CO}_{2}^{-}$ and $\mathrm{NH}_{3}^{+}$groups. In a polar crystal the structure of the opposite surfaces within a crack, perpendicular to 
the polar axis, are radically different. Were such a surface to carry a net charge, the two opposite faces would carry charges of opposite sign. In contrast, the opposite faces within a crack of a centrosymmetric crystal have similar structures with no net charge on each of the two faces. Therefore, it was deduced that the opposite faces of cracks along the polar axis produced an electric field strong enough for the alignment of water molecules into proton-ordered ice clusters which would be polar along its hexagonal axis [Fig. 35(b)]. According to this model it was predicted and shown for alanine and tyrosine, whose racemic crystals have a polar axis and whose chiral counterparts do not, that the racemic crystal induces ice nucleation at temperatures higher by $c a 5 \mathrm{~K}$. This model is in agreement with calculations of interaction energy between polar and nonpolar hexagonal ice-like clusters contained within the crevice of both $(R, S)$ and $(S)$ of alanine (Lahav, Eisenstein \& Leiserowitz, 1993). The ice freezing point results were explained on a more macroscopic level by Wilen (1993), who invoked the role of the difference in dielectric constants of water and ice.

We have already alluded to the fact that several strains of bacterial species, such as pseudomonas syringae, can catalyze ice nucleation at temperatures as high as $271 \mathrm{~K}$.

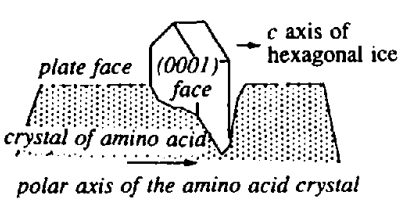

(a)

Plate face of polar crystal

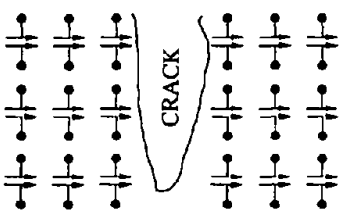

1. Hydrophobic residue

Hydropholic $\mathrm{NH}_{3}^{+} \mathrm{CHCO}_{2}^{-}$group

(c)

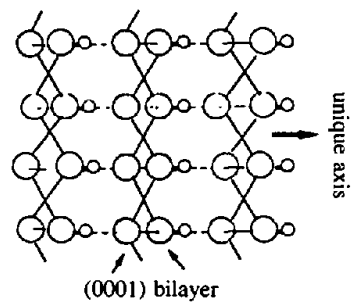

(b)

Plate face of nonpolar crystal

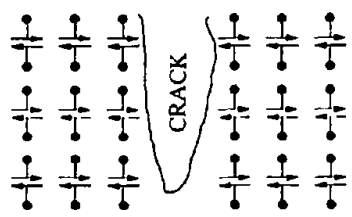

(d)
Fig. 35. (a) Schematic view of hexagonal ice crystals (exaggerated for clarity) emerging from the plate face of an $\alpha$-amino acid crystal. (b) Molecular packing of a hexagonal ice from which the $\mathrm{O}-\mathrm{H} \cdots \mathrm{O}$ hydrogen bonds along the unique axis are assumed to be proton-ordered and so the structure is polar along this direction. The remaining $\mathrm{O}-\mathrm{H} \cdots \mathrm{O}$ bonds per molecule within each $(0001)$ bilayer are assumed to be proton-disordered. (c) Schematic view of a polar $\alpha$-amino acid crystal composed of hydrogen-bonded bilayers. The polar axis is along the horizontal direction. The opposite faces within the cracks expose different groups. (d) Schematic view of the centrosymmetric $\alpha$-amino acid crystal. The opposite faces within the crack are equivalent.
The membrane proteins responsible for ice nucleation have been sequenced (Green \& Warren, 1985; Warren, Corotto \& Wolber, 1986; Warren \& Corotto, 1989). It was recognized by Warren and coworkers (1986) that the protein probably consists largely of $\beta$-strands. They proposed a schematic arrangement having a triangular shape similar to that of the $a b$-layer of hexagonal ice. Mizuno (1989) proposed a more detailed model. Kajava \& Lindow (1993) pointed out some disadvantages of these models: the lack of close packing of the protein structures as well as the inability of the water molecules at the ice-like template to form all necessary hydrogen bonds. They have presented a structural model which consists of a largely planar extended macromolecule with one side interacting with the membrane, and the other side serving as a template for orienting water into an ice lattice. Such ice nuclei would not be formed from an $a b$-layer so that an experimental determination of the oriented ice crystallites could shed light on the validity of the model.

\section{Conclusions}

A great variety of crystalline properties can be controlled and modified with the use of tailor-made auxiliaries, including growth and dissolution rates, morphology, crystal texture, etch-pit formation, structure and polymorphism. Surprisingly, such studies also allow one to help elucidate basic scientific questions, such as pinpointing fine molecular interactions at interfaces, the correlation between molecular chirality and macroscopic phenomena in crystals and the role played by solvent on crystal growth and morphology. Control of crystal formation in the very early stages is still primitive and the monitoring of such a process by diffraction methods is still to be done. Very little work has been done with tailor-made additives on liquid crystals or on crystals composed of macromolecules, such as proteins. Indeed one has only to look at the fine control achieved in biomineralization to realize that we still have a long way ahead.

This review describes work carried out over the past decade. We wish to acknowledge the contributions of L. Addadi and Z. Berkovitch-Yellin. We also thank all students and post-doctoral fellows who were engaged in the work and whose names are listed in the references. $\mathrm{X}$-ray diffraction measurements on solid materials were performed together with F. Frolow and neutron diffraction studies with R. K. McMullan of the Brookhaven National Laboratory. The grazing incidence synchrotron $\mathrm{X}$-ray diffraction measurements on the monolayers were carried out at DESY, Hamburg, in a fruitful collaboration with K. Kjaer and J. Als-Nielsen of Riso National Laboratories, Denmark. We thank J. M. McBride and $P$. Bennema for a critical reading of the manuscript. 
This work was supported over the years by the Minerva Foundation, Münich, the Israel Academy of Sciences, the US-Israel Binational Science Foundation, The Petroleum fund of the American Chemical Society and the German-Israeli Foundation.

\section{References}

Addadi, L. \& Lahav, M. (1979). Pure Appl. Chem. 51, 1269-1284. ADDADI, L. \& WEINER, S. (1992). Angew. Chem. Int. Ed. Engl. 31, 153-169.

AdDadi, L., ARIEl, S., Lahav, M., Leiserowitz, L., Popovttz-BIRo, R. \& Tang, C. P. (1980). In Chemical Physics of Solids and their Surfaces, Vol. 8, p. 202. Specialist Periodical Reports.

AdDadi, L., BerkovtTCh-Yellin, Z., DomB, N., Gati, E., Lahav, M. \& LEISEROWTTZ, L. (1982). Nature, 296, 21-26.

AdDADI, L., Berkovitch-Yellin, Z., Weissbuch, I., Lahav, M. \& LEISEROWTTZ, L. (1986). In Topics in Stereochemistry, edited by E. L. Eliel, S. H. Wlllen \& N. L. Allinger, Vol. 16, pp. 1-85. New York: John Wiley \& Sons, Inc.

ADDADI, L., BERKovtTCH-Yellin, Z., WeIsSBuch, l., van Mil, J., SHMON, L. J. W., LahaV, M. \& Leiserowitz, L. (1985). Angew. Chem. Int. Ed. Engl. 24, 466-485.

AdDadi, L., van MII, J. \& LaHav, M. (1981). J. Am. Chem. Soc. 103. $1249-1251$.

AdDadi, L., VAn MII, J. \& Lahav, M. (1982). J. Am. Chem. Soc. 104, 3422-3429.

Addadi, L., Weinstein, S., Gati, E., Weissbuch, I. \& Lahav, M. (1982). J. Am. Chem. Soc. 104, 4610-4617.

Allen, F. M. \& BuseCK, P. R. (1988). Am. Miner. 73, 568.

Als-NiElSEN, J. \& KJaER, K. (1989). In The Proceedings of the NATO Advanced Study Institute, Phase Transitions in Soft Condensed Matter, Series B, Vol. 211, p. 113. New York, London: Plenum Press.

ALS-NIELSEN, J. \& MOHWALD, H. (1992). In Handbook of Synchrotron Radiation, Vol. 4. Amsterdam: North Holland.

Antinozzi, P. A., Brown, C. M. \& Purich, D. L. (1992). J. Cryst. Growth, 125, 215-222.

BARTHAKUR, N. \& MAYBANK, DT J. (1963). Nature, 200, 866-868.

Barton, S. W., Goudot, A., Bouloussa, O., Rondelez, F., LiN, B., Novak, F., Acero, A. \& Rice, S. (1992). J. Chem. Phys. 96, 1343-1351.

Barton, S. W., Thomas, B. N., Flom, E. B., Rice, S., Lin, B., Peng, J. B., Ketterson, J. B. \& Dutta, P. (1988). J. Chem. Phys. 89, 2257-2270.

Benedetti, E., Pedone, C. \& Sirigu, A. (1973). Acta Cryst. B29, 730-733.

BenNeMA, P. (1992). J. Cryst. Growth, 122, 110-119.

BenNema, P. \& GILMER, G. (1973). In Crystal Growth, An Introduction edited by P. HARTMAN, p. 272. Amsterdam: North-Holland.

Berkovitch-Yellin, Z. (1985). J. Am. Chem. Soc, 107, 8239-8253.

BerKovitCH-YelliN, Z. \& LeISEROWTtz, L. (1984). Acta Cryst. B40, $159-165$.

Berkovitch-Yellin, Z., Addadi, L., Idelson, M., Lahav, M. \& Leiserowitz, L. (1982). Angew. Chem., Suppl., pp. 1336-1345.

BerkovttCh-Yellin, Z., ADDADi, L., IDEISON, M., LeiserowitZ, L. \& LAHAV, M. (1982). Nature, 296, 27-34.

BERKOVITCH-Yellin, Z., VAN Mill, J., AdDADI, L., IDELSON, M., LAHAV, M. \& LEISEROWTT, L. (1985). J. Am. Chem. Soc. 107, 3111-3!22.

BeRKovitch-Yellin, Z., Weissbuch, I. \& Leiserowitz, L. (1989). In Morphology and Growth Units of Crystals, p. 247. Tokyo: Terrapub.

Berman, A., Addadi, L., KVick, A., Leiserowitz, L., Nelson, M. \& WEINER, S. (1990). Science, 250, 664-667.

Berman, A., Hanson, J., Leiserowitz, L., Koetzle, T. F., Weiner, S. \& ADDADI, L. (1993a). Science, 259, 776-779.

Berman, A., Hanson, J., Leiserowitz, L., Koetzle, T. F., Weiner, S. \& ADDADI, L. (1993b). J. Phys. Chem. 97, 5162-5170.

Black, S. N., Bromley, L. A., CotTIER, D., Davey, R. J., Dobbs, B. \& RouT, J. E. (1991). J. Chem. Soc. Faraday Trans. 87, 3409-34I4.

BoEk, E. S. (1993). Crystal-solution interfaces. PhD Thesis. Univ. of Twente, The Netherlands.

BoEk, E. S. \& BrIEls, W. J. (1992). J. Chem. Phys. 96, 7010-7018.
Boek, E. S., Fell, D., Briels, W. J. \& Bennema, P. (1991). J. Cryst. Growth, 114, 389-410.

BOER, R. C. DE (1993). Morphology related phenomena. PhD Thesis. Catholic Univ. of Nijmegen, The Netherlands.

Bohm, C., Levenler, F., Jacquemain, D., Möhwald, H., Kuaer, K., Als-NIELSEN, J., WeISSBUCH, I. \& LeISEROWTT, L. (1994). Langmuir, 10, 830-836.

BourNe, J. R. \& DaveY, R. J. (1976). J. Cryst. Growth, 36, 278-286.

BUCKLEY, H. E. (1951). Crystal Growth. New York: Wiley.

Chakrabartty, A., Yang, D. S. C. \& Hew, C. L. (1989). J. Biol. Chem. 264, 11313-11316.

Chen, B, D., GaRside, J., Davey, R. J., Maginn, S. J. \& Matsuoka, M. (1994). J. Phys. Chem. 98, 3215-3221.

Chiarello, R. P., Wogelins, R. A. \& Sturchuo, N. C. (1993). Geochim. Cosmochim. Acta, 57, 4103-4110.

CoDy, A. M. \& CoDY, R. D. (1991). J. Cryst. Growth, 113, 508-519.

Corrin, M. L. \& Nelson, J. A. (1968). J. Phys. Chem. 72, 643-645.

Cunningham, D. A. H., Gerson, A. R., Roberts, K. J., Sherwood, J. N. \& WoJCIECHOWSKI, K. (1991). In Advances in Industrial Crystallization, edited by J. GARSIDE, R. J. DaVEY \& A. G. JonES, p. 105. Oxford: Butterworth-Heinemann Ltd.

CurIe, M. P. (1894). J. Phys. p. 393.

DaveY, R. J., Milisavlevic, B. \& BolRne, J. R. (1988). J. Phys. Chem. 92, 2032-2036.

DE VRIES, A. L. (1984). Phil. Trans. R. Soc. 304, 575-588.

DOCherty, R., RoberTS, K., SAUNDers, V., Black, S. \& Davey, R. J. (1993). Faraday Discuss. 95, 11-26.

Dutta, P., Peng, J. B., Lin, B., Ketterson, J. B., Prakash, M., Geogopolros, P. \& ERLJCH, S. (1987). Phys. Rev. Lett. 58, 2228-2231.

Elwenspoek, M., Bennema, P. \& van Eerden, J. P. (1987). J. Cryst. Growth, 83, 297-305.

Feeney, R. E., Burcham, T. S. \& YeH, Y. (1986). Ann. Rev. Biophys. Biophys. Chem. 15, 59-78.

FENDLER, J. (1993). Private communication.

Fentmore, C. P. \& Thraikll, A. (1949). J. Am. Chem. Soc. 71, 2714-2717.

Fletcher, N. H. (1970). In The Chemical Physics of Ice. Cambridge Univ. Press.

Gavish, M., Popovitz-Biro, R., LaHav, M. \& Leiserowitz, L. (1990). Science, 250, 973-975.

Gavish, M., Wang, J. L., Eisensten, M., Lahav, M. \& Leiserowttz, L. (1992). Science, 256, 815-818.

Gevers, R., Amelinckx, S. \& Dekeyser, W. (1952). Naturwissenschaften, 39, 448-449.

Gidalevitch, D., Weissbuch, I., KJaer, K., Als-Nielsen, J. \& LeISEROWITZ, L. (1994). J. Am. Chem. Soc. 116, 3271-3278.

Gopalan, P., Peterson, M. L., Crundwell, G. \& Kahr, B. (1993). J. Am. Chem. Soc. 115, 3366-3367.

GREEN, R. L. \& WARREN, G. J. (1985). Nature, 317, 645-648.

Green, B. S., Lahav, M. \& Rabinovich, D. (1979). Acc, Chem. Res. 12, $191-197$.

HARTMAN, P. (1973). In Crystal Growth. An Introduction, p. 367. Amsterdam: North-Holland Publishing Co.

Hartman, P. \& Bennema, P. (1980). J. Cryst. Growth, 49, 145-165.

Hartman, P. \& Perdok, W. G. (1955a). Acta Cryst. 8, 49-52.

Hartman, P. \& Perdok, W. G. (1955b). Acta Cryst. 8, 525-529.

HEYWOOD, B. R. \& MANN, S. (1992a). Langmuir, 8, 1492-1498.

HeYwOOD, B. R. \& MANN, S. (1992b). J. Am. Chem. Soc. 114, $4681-4686$.

HiRshFeld, F. L. (1977). Theor. Chem. Acta, 44, 129-138.

HONESS, A. P. (1927). The Nature, Origin and Interpretation of the Etch Figures on Crystals. New York: Wiley.

HoOK, A. VAN (1968). Kristal. Acad. Nauk. SSSR Inst. Kristallogr. 8. 45.

HOOK, A. VAN (1981). J. ASSBT, 21, 130.

HoRN, F. H. (1952). Philos. Mag. 43, 1210-1213.

IITAKA, Y. (1961). Acta Cryst. 14, 1-10.

JaCquemain, D., Grayer Wolf, S., LeVeiller, F., Deutsch, M.. KJaer, K., Als-Nielsen, J., Lahav, M. \& Leiserowitz, L. (1992). Angew. Chem. Inl. Ed. Engl. 31, 130-152.

Jacquemain, D., Grayer Wolf, S., Leveiller, F., Lahav, M. Leiserowitz, L., Deutsch, M., KJAER, K. \& Als-Nielsen, J. (1990). J. Am. Chem. Soc. 112, 7724-7736. 
JaCQueMain, D., LeVELleR, F., WeinBach, S., LAHAV, M., Leiserowitz L., KJaer, K. \& Als-Nielsen, J. (1991). J. Am. Chem. Soc. 113, 7684-7691.

Jonsson, P. G. (1971). Acta Cryst. B27, 893-898.

Kajava, A. V. \& Lindow, S. E. (1993). J. Mol. Biol. 232, 709-717.

KERN, R. (1953). Bull. Soc. Fr. Mineral. Cristallogr. 76, 391.

Knight, C. A., Chang, C. C. \& De VrIes, A. L. (1991). Biophys. J. 59, 409-418.

Lahav, M., Eisenstein, M. \& Leiserowitz, L. (1993). Science, 259. 1469-1470.

Landau, E. M., Popovitz-Biro, R., Levanon, M., Leiserowitz, L. \& Lahav, M. (1986). Mol. Cryst. Liq. Cryst. 134, 323-335.

LANDAU, E. M., Wolf, S. G., LeVANON, M., LeISEROWTTZ, L., LAHAV, M. \& Sagiv, J. (1989). J. Am. Chem. Soc. 111, 1436-1445.

LeVElller, F., BoEhm, C., JacQuemain, D., Mohwald, H., LeiserowtTZ, L., KJAER, K. \& ALs-NIELSEN, J. (1994). Langmuir, 10, 819-829.

LeVeIller, F., Jacquemain, D., Lahav, M., LeiserowtTZ, L., Deutsch, M., KJAER, K. \& ALS-NIELSEN, J. (1991). Science, 252, 1532-1536.

LeVEILleR, F., Jacquemain, D., Leiserowitz, L., KJaER, K. \& AlsNielsen, J. (1992). J. Phys. Chem. 96, 10380-10389.

Lewtas, K., Tack, R. D., BeinY, D. H. M. \& Mullin, J. W. (1991). Advances in Industrial Crystallization, p. 166. Oxford: ButterworthHeinemann L.td.

LINDOw, S. E. (1983). Annu. Rev. Phytopathol 21, 363-384.

LINDOw, S. E., ARNY, D. C. \& UPPER, C. D. (1978). Phytopathology, $68,523-527$.

Löwenstam, H. A. \& WeINER, S. (1989). On Biomineralization. New York: Oxford Univ. Press.

Majewski, J., Edgar, R., Popovttz-BrRo, R., BouWman, W. G., KJaER, K., Als-Nielsen, J., Lahav, M. \& LeiserowtTz, L. (1995). Angew. Chem. In the press.

Majewski, J., Margulis, L., Jacguemain, D., Levenler. F., Böhm. C., Arad, T., Talmon, Y., Lahav, M. \& Leiserowitz, L. (1993). Science, 261, 899-902.

Majewsk, J., Popovitz-Biro, R., Kuaer, K., Als-Nielsen, J., Lahav, M. \& Leiserowitz, L. (1994). J. Phys. Chem. 98, 4087-4093.

MaJewsk, J., PopovtTz-Biro, R., Boumman, W. G., KJaEr, K., AlsNielsen, J., LahaV, M. \& Leiserowitz, L. (1995). Chem. Eur. J. Submitted.

Maki, L. R., Galyan, E. L., Chang Chien, M. \& Caldwell, D. R. (1974). Appl. Microbiol. 28, 456-459.

Mantovani, G., Gilli, G. \& Faglioli, F. (1967). Proc. 13th Conf. CITS, Falsterbo, Sweden, pp. 289-301.

MANN, S. (1988). Nature, 332, 119-124.

ManN, S., HeYwoOd, B. R., Rajam, S. \& Birchall, J. D. (1988), Nature, 334, 692-695.

Markman, O., Elias, D., Addadi, L., Cohen, I. R. \& BerkovitchYelLn, Z. (1992). J. Cryst. Growth, 122, 344-350.

McBride, J. M. \& Bertman, S. B. (1989). Angew. Chem. Int. Engl. 28 330-333.

Michaelis, A. S. \& VAN KReveld, X. (1966). Neth. Milk Dairy J. 20, 163-181.

Mil, J. van, Addadi, L., Gati, E., \& LaHav, M. (1981). J. Am. Chem. Soc. 103, 1248-1249.

Mil, J. van, AdDadi, L., Gati, E. \& LaHav, M. (1982). J. Am. Chem. Soc. 104, 3429-3434.

Mizuno, H. (1989). Proteins: Struct. Funct. Genet. 5, 47-65.

Ocko, B. M., Wang, J., DavenPort, A. \& IsaAcs, H. (1990). Phys. Rev. Lett. 65, 1466-1469.

Parungo, F. P. \& Lodge, J. P. JR. (1967). J. Atm. Sci. 24, 274-277.

Paul, I. C. \& CurTin, D. Y. (1987). Organic Solid State Chemistry, p. 331. Amsterdam: Elsevier.

Popovitz-Biro, R., Majewski, J., Margulis, L., Cohen, S., Leiserowitz, L. \& Lahav, M. (1994a). J. Phys. Chem. 98, 4970-4972.

Popovitz-Biro, R., Majewski, J., Margulis, L., Cohen, S., Leiserowitz, L. \& LaHav, M. (1994b). Adv. Mat. 6, 956-959.

Popovitz-Biro, R., Wang, J. L., MaJeWski, J., Leiserowitz, L. \& LAHAV, M. (1994). J. Am. Chem. Soc. 116, 1179-1191.

POWER, B. A. \& POWER, F. (1962). Nature, 194, 1170-1171.

Prappacher, H. R. (1963). Z. Angew. Math. Phys. 14, 590-598.

Prelog, V. \& Helmchen, G. (1982). Angew. Chem. Int. Ed. Engl. 21, $567-583$.
Ristic, R. I., Sherwood, J. M. \& WoJeshowsKI, K. (1994). J. Phys. Chem. In the press.

SCHNELl, R. C. \& VALI, C. (1972). Nature, 236, 163-165.

SHMON, L. J. W., LAHAV, M. \& LeISEROWTTZ, L. (1985). J. Am. Chem. Soc. 107, 3375-3377.

Shimon, L. J. W., Lahav, M. \& Leiserowitz, L. (1986). Nouv. J. Chem. 10, 723-737.

ShImon, L. J. W., VadDa, M., AdDadi, L., LaHAV, M. \& Leiserowitz, L. (1990). J. Am. Chem. Soc. 112, 6215-6220.

SHImon, L. J. W., VAIDA, M., AdDadi, L., LAHAV, M. \& LeISEROWITZ, L. (1991). In Organic Crystal Chemistry, p. 74. Oxford Univ. Press.

Shimon, L. J. W., Vaida, M., Frolow, F., Lahav, M., Leiserowitz, L., Weissinger-LeWI, Y. \& MCMulLaN, R. K. (1993). Faraday Discuss. $95,307-327$.

Shimon, L. J. W., Wireko, F. C., Wolf, J., Weissbuch, I., ADDadi, L., BerkovitCh-YellIN, Z., LAHAV, M. \& LeISEROWITZ, L. (1986). Mol. Cryst. Liq. Cryst. 137, 67-86.

Shimon, L. J. W., Zbaida, D., AdDadi, L., Leiserowitz, L. \& Lahay, M. (1988). Mol. Cryst. Liq. Cryst. 161, 199-221.

Skoulika, S., Michaelidis, A. \& Aubry, A. (1991). J. Cryst. Growth, 108, 285-289.

SMYTHE, B. M. (1967). Austr. J. Chem. 20, 1115-1131.

SMYTHE, B. M. (1971). Sugar Technol. Rev. 1, 191-231.

STAAB, E., ADDADI, L., LeISEROWITZ, L. \& LAHAV, M. (1990). Adv. Mater. 2, 40-43.

Swaminathan, K. S. \& Craven, B. M. (1982). Am. Cryst. Assoc. Ser. $2,10,13$.

TaYlor, R. \& Kennard, O. (1982). J. Am. Chem. Soc. 104, 5063-5070.

TORRI, K. \& YITAKA, Y. (1970). Acta Cryst. B26, 1317-1326.

TORR1, K. \& YITAKA, Y. (1971). Acta Cryst. B27, 2237-2246.

Turner, E. \& Lonsdale, K. (1950). J. Chem. Phys. 18, 156.

Vaida, M., Shtmon, L. J. W., van Mil, J., Ernst-Cabrera, K., AdDadi, L., Leiserowitz, L. \& Lahav, M. (1989). J. Am. Chem. Soc. 111, 1029-1034.

Vaida, M., Shimon, L. J. W., Weisinger-Lewin, Y., Frolow, F., Lahav, M., Leiserowitz, L. \& MCMUllaN, R. K. (1988). Science, 241, 1475-1479.

VisSER, R. A. \& BENNEMA, P. (1983). Neth. Milk Dairy J. 37, 109-137. VONNEgUT, B. (1947). J. Appl. Phys. 18, 593-595.

Walba, D. M., Razuvi, H. A., Clark, N. A. \& Parmer, D. S. (1988) J. Am. Chem. Soc. 110, 8686-8691.

WANG, J., DavenPoRT, A., ISAaCs, H. \& Ocko, B. M. (1992). Science, 255, 1416-1418.

Wang, J.-L., LaHAV, M. \& Lejserowitz, L. (1991). Angew. Chem. Int. Ed. 30, 696-698.

Wang, J.-L., Leiserowitz, L. \& Lahav, M. (1992). J. Phys. Chem. 96, $15-16$.

Wang, J.-L., Levelller, F., Jacquemain, D., Kiaer, K., Als-Niel.sen, J., Lahav, M. \& Leiserowitz, L. (1994). J. Am. Chem. Soc. 116, 1192-1204.

WARREN, G. J. \& CoRotTo, L. (1989). Gene, 85, 239-243.

WARREN, G. J., CoRotTo, L. \& Wolber, P. (1986). Nucl. Acids Res. 14, 8047-8060.

Weinbach, S., Jacquemain, D., LeVeller, F., KJaer, K., Als-Nielsen, J. \& LeISEROWITZ, L. (1993). J. Am. Chem. Soc. 115, 11110-11118.

Weinbach, S., Kuaer, K., Als-Nielsen, J., Grubel, G., Legrand, M. LAHAV, M. \& LeISERowitz, L. (1994). Science, 264, 1556-1570.

Weissbuch, I., Addadi, L., Berkovitch-Yellin, Z., Gati, E., Lahav, M. \& LeISEROWITZ, L. (1984). Nature, 310, 161-164.

Weissbuch, l., AdDadi, L., Berkovitch-Yellin, Z., Gati, E., Weinstein, S., Lahav, M. \& Leiserowitz, L. (1983). J. Am. Chem. Soc. 105. 6613-6621.

WeISSBuch, I., ADDadi, L., Lahav, M. \& LeISERowitz, L. (1991) Science, 253, 637-645.

Weissbuch, I., AdDadi, L., Leiserowitz, L. \& Lahav, M. (1988). $J$. Am. Chem. Soc. 11, 561-567.

Weissbuch, I.. Berkovic, G.. Leiserowitz, I.. \& Lahav, M. (1990). J. Am. Chem. Soc, 112, 5874-5875.

Weissbuch, I., Berkovttch-Yellin, Z., Leiserowitz, L. \& Lahav, M. (1985). Isr. J. Chem. 25, 362-372.

Weissbuch, I., Frolow, F., AdDadi, L., Lahav, M. \& Leiserowttz, L. (1990). J. Am. Chem. Soc. 112, 7718-7724. 
WeisSBuCh, I., KUZMENKo, I., VAIDA, M., ZAT, S., LeISEROWITZ, L. \& LAHAV, M. (1994). Chem. Mater. 6, 1258-1268.

Weissbuch, I., Lahav, M., Leiserowitz, L., Meredith, G. R. \& VANHERZEELE, H. (1989). Chem. Mater. 1, 114-118.

WeissbuCH, I., Leiserowttz, L. \& LAHAV, M. (1994). Adv. Mat. 6, 953-956.

Weissbuch, I., Leveiller, F., Jacquemain, D., KJaer, K., Als-Nielsen, J. \& Leiserowitz, L. (1993). J. Phys. Chem. 97, 12858-12861.

Weissbuch, I., Majewski, J., KJaer, K., Als-Nielsen, J., Lahav, M. \& LeiserowTt, L. (1993). J. Phys. Chem. 97, 12848-12857.

WeIsSBUCh, I., SHIMON, L. J. W., ADDADI, L., BERKOVITCH-Yellin, Z., Weinstein, S., Lahav, M. \& Leiserowttz, L. (1985). Isr. J. Chem. 25, 353-362.

Weissbuch, I., Zbaida, D., Addadi, L., Lahav, M. \& Leiserowitz, L. (1987). J. Am. Chem. Soc. 109, 1869-1871.

Weissinger-Lewin, Y., Frolow, F., McMullan, R. K., Koetzle, T. F., Lahav, M. \& Leiserowitz, L. (1989). J. Am. Chem. Soc. 111, 1035-1040.
Wells, A. F. (1946). Philos. Mag. 37, 180, 217, 605-629.

WELLS, A. F. (1949). Discuss. Faraday Soc. 5, 197-201.

WHETSTONE, J. (1954). Faraday Discuss. pp. 132-140.

WILEN, L. (1993). Science, 259, 1469.

Wireko, F. C., Shmon, L. J. W., Frolow, F., Berkovttch-Yellin, Z., Lahav, M. \& Lelserowttz, L. (1987). J. Phys. Chem. 91, 472-481.

WOENSDREGT, C. F. (1993). Faraday Discuss. 95, 97-108.

Wolf, S. G., Leiserowitz, L., Lahav, M., Deutsch, M., Kuaer, K. \& ALS-NIELSEN, J. (1987). Nature, 328, 63-66.

Yang, D. S. C., Sax, M., ChakrabartTy, A. \& Hew, C. L. (1988). Nature, 333, 232-237.

Zabel, V., Muller-Fahrnow, M., Hilgenfeld, R., Saenger, W., Pfannemuller, B., Enkelmann, V. L. \& Welke, W. (1986). Chem. Phys. Lipids, 39, 313-327.

Zbaida, D., Weissbuch, 1., Shavit-Gati, E., Addadi, L., Leiserowitz, L. \& LaHav, M. (1987). React. Polym. 6, 241-253.

ZhaO, X. K., Yang, J., MCCORMick, L. D. \& FendleR, J. H. (1992). J. Phys. Chem. 96, 9933-9939. 